\title{
Branching Variable Direction Selection in Mixed
}

\section{Integer Programming}

by

\author{
Jennifer Pryor, B. Eng.
}

A thesis submitted to the Faculty of Graduate Studies and Research in partial fulfillment of the requirements for the degree of

\section{Master of Applied Science (M.A. Sc.) in Electrical Engineering}

\author{
Ottawa-Carleton Institute of Electrical and Computer Engineering \\ Faculty of Engineering \\ Department of Systems and Computer Engineering \\ Carleton University \\ Ottawa, Ontario, K1S 5B6, Canada.
}

June 18, 2009 


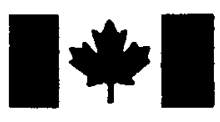

\author{
Library and Archives \\ Canada \\ Published Heritage \\ Branch \\ 395 Wellington Street \\ Ottawa ON K1A ON4 \\ Canada
}

Bibliothèque et

Archives Canada

Direction du

Patrimoine de l'édition

395, rue Wellington

Ottawa ON K1A 0N4

Canada
Your file Votre référence
ISBN: 978-0-494-60229-4
Our file Notre reference
ISBN: $978-0-494-60229-4$
NOTICE:

The author has granted a nonexclusive license allowing Library and Archives Canada to reproduce, publish, archive, preserve, conserve, communicate to the public by telecommunication or on the Internet, loan, distribute and sell theses worldwide, for commercial or noncommercial purposes, in microform, paper, electronic and/or any other formats.

The author retains copyright ownership and moral rights in this thesis. Neither the thesis nor substantial extracts from it may be printed or otherwise reproduced without the author's permission.

\section{AVIS:}

L'auteur a accordé une licence non exclusive permettant à la Bibliothèque et Archives Canada de reproduire, publier, archiver, sauvegarder, conserver, transmettre au public par télécommunication ou par l'Internet, prêter, distribuer et vendre des thèses partout dans le monde, à des fins commerciales ou autres, sur support microforme, papier, électronique et/ou autres formats.

L'auteur conserve la propriété du droit d'auteur et des droits moraux qui protège cette thèse. Ni la thèse ni des extraits substantiels de celle-ci ne doivent être imprimés ou autrement reproduits sans son autorisation.
In compliance with the Canadian Privacy Act some supporting forms may have been removed from this thesis.

While these forms may be included in the document page count, their removal does not represent any loss of content from the thesis.
Conformément à la loi canadienne sur la protection de la vie privée, quelques formulaires secondaires ont été enlevés de cette thèse.

Bien que ces formulaires aient inclus dans la pagination, il n'y áura aucun contenu manquant.

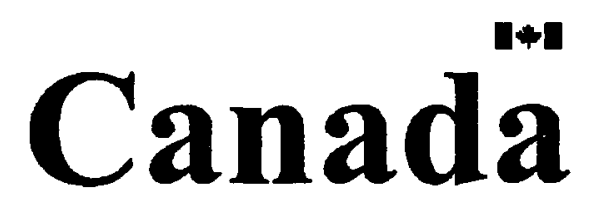




\section{Abstract}

Many real-world applications can be modeled as Mixed Integer Programming (MIP) problems. Branch and Bound $(\mathrm{B} \& \mathrm{~B})$ is a common algorithm used to solve MIPs. Three selections are made during the B\&B algorithm: branching variable, branching direction, and node. To date no empirical studies have been conducted on which branching direction heuristic works best, yet it has a significant impact on solution speed. The focus of this thesis is to conduct such a study. New branching direction methods are proposed, using the probability a given variable-value combination will satisfy a constraint. Branching up is confirmed as the best branching direction heuristic to find the first integer-feasible solution. The probability methods are extended to select both the branching variable and direction. It results in a heuristic that reaches first feasibility faster than the GLPK 4.28 [Makhorin 2008] heuristics. This method compares well with the state of the art when the MIP has at least one equality constraint. 


\section{Acknowledgements}

I would like to thank my thesis supervisor, Professor John Chinneck (Systems and Computer Engineering Department, Carleton University) for his insight, reassurance, and guidance in this research. I would also like to thank Darlene Hebert and the rest of her staff (Systems and Computer Engineering Department, Carleton University) for their administrative assistance whenever I required it. I would like to thank Narendra Mehta, Danny Lemay, and the rest of the Technical Staff (Systems and Computer Engineering Department, Carleton University) for their effective assistance with computing resources and lab set-up. I am thankful to Andrew Makhorin (Department for Applied Informatics, Moscow Aviation Institute, Moscow, Russia) for creating and maintaining GLPK [Makhorin 2008] with solid documentation and accessible help forums. I would like to thank Dan Wojtaszek (Systems and Computer Engineering Department, Carleton University) for his support on GLPK [Makhorin 2008]. Finally, I would like to thank my family and friends for their immeasurable support and encouragement during my studies. 


\section{Table of Contents}

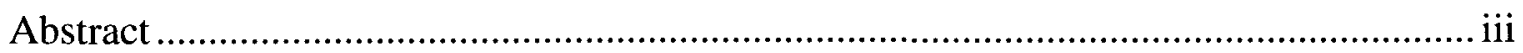

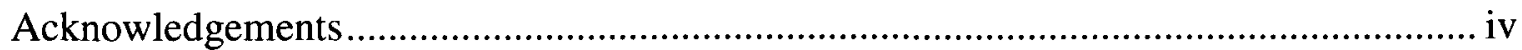

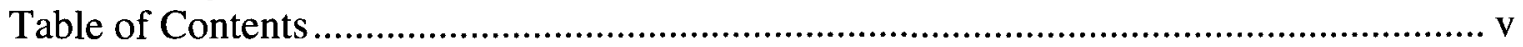

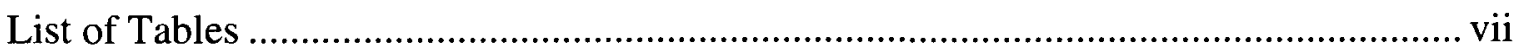

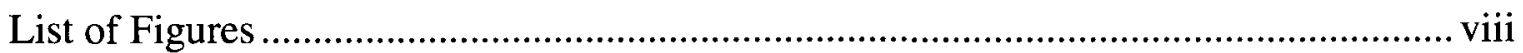

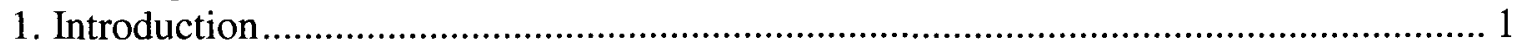

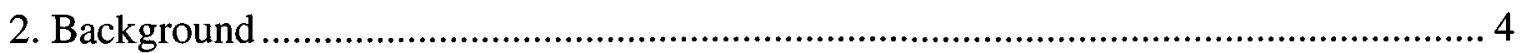

2.1 Using the Branch and Bound Method to Solve MIPs........................................... 4

2.2 Counting Solutions of Knapsack Constraints ...................................................... 10

2.3 Performance Profiles............................................................................... 15

2.4 Branching Variable Selection Algorithms ..................................................... 18

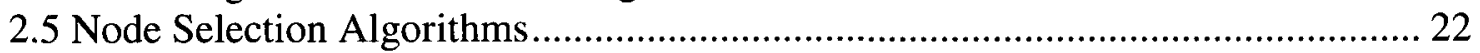

3. State of the Art in Branching Variable Direction Selection ......................................... 25

3.1 Simple Branching Variable Direction Heuristics ................................................... 25

3.2 Studies Discussing Branching Variable Direction Selection.................................. 26

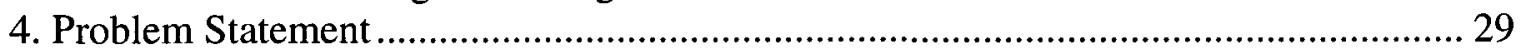

5. New Branching Variable Direction Selection Heuristics ............................................. 31

5.1 Extension of Counting Solutions Methodology for Direction Selection ................. 31

5.1.1 Branching Direction Methods Using Cumulative Probabilities ....................... 34

5.2 Satisfied and Violated Voting Methods................................................................ 37

5.2.1 Branching Direction Methods Using Violated and Satisfied Voting Methods 40

5.3 Extension of Counting Solutions Methodology for Simultaneous Variable and

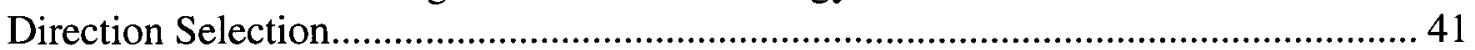

5.3.1 Simultaneous Variable and Direction Selection Methods Using Cumulative

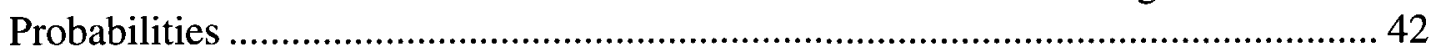

5.3.2 Simultaneous Variable and Direction Selection Methods Using Point Probabilities

5.3.3 Simultaneous Variable and Direction Selection Methods Using Multiplied Probabilities

5.3.4 Simultaneous Variable and Direction Selection Methods Using Hybrid Methods

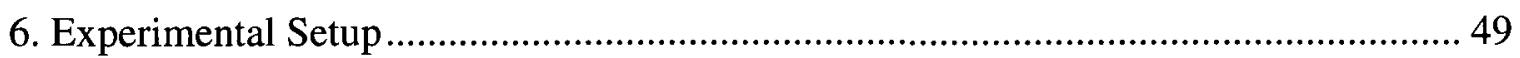

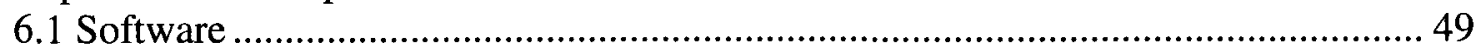

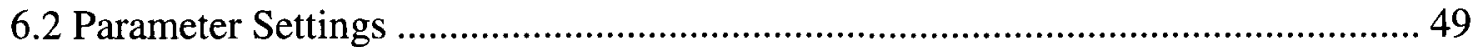

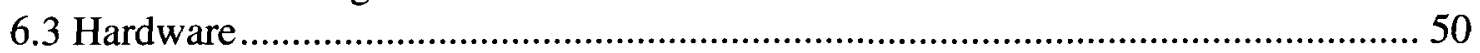

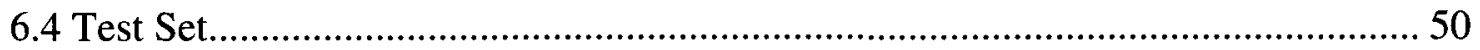

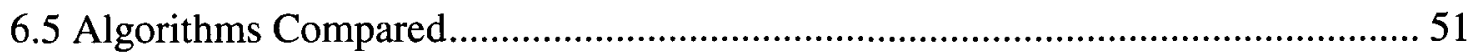

6.6 Performance Metrics ........................................................................................ 52

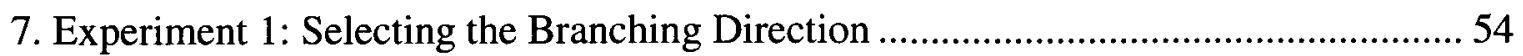

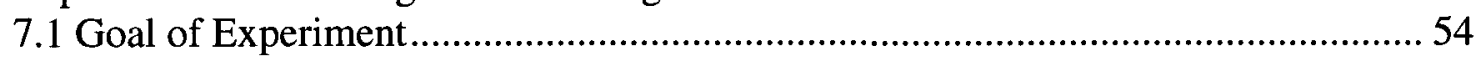

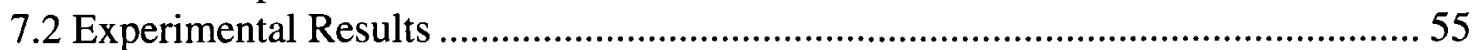


8. Experiment 2: Simultaneous Selection of Branching Variable and Direction ............. 72

8.1 Cumulative and Point Probability Methods .......................................................... 72

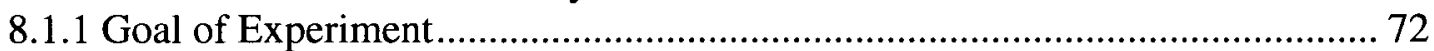

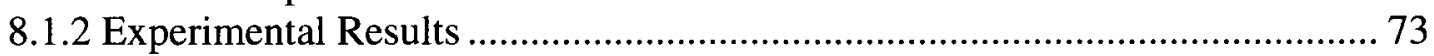

8.2 Multiplied Probability Methods............................................................................. 75

8.2.1 Goal of Experiment .................................................................................. 75

8.2.2 Experimental Results .................................................................................. 75

8.3 Equality-Free versus Equality-Containing MIPs .................................................. 77

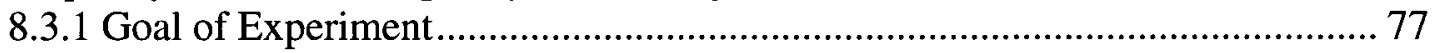

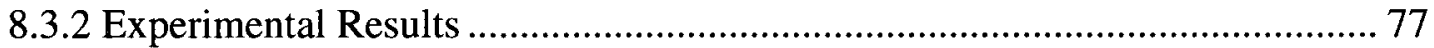

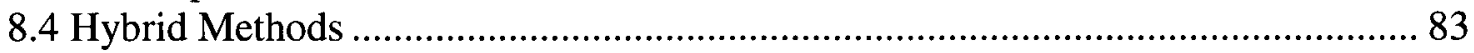

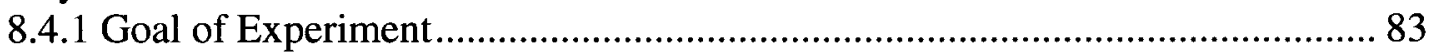

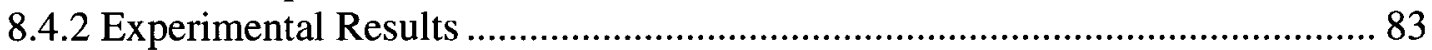

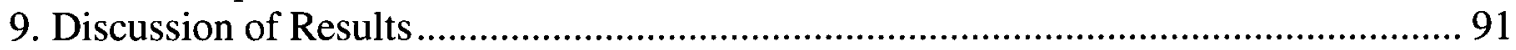

9.1 Role of Multiple Choice Constraints .................................................................. 91

9.2 Number of Violated Constraints in MIPs ......................................................... 99

9.3 Focusing on Hardest Constraints .................................................................. 102

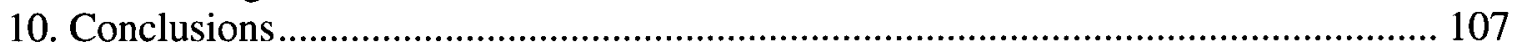

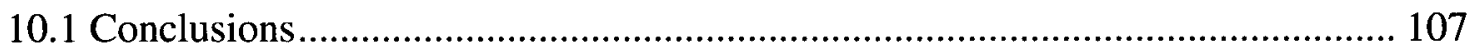

10.2 Summary of Contributions............................................................................ 108

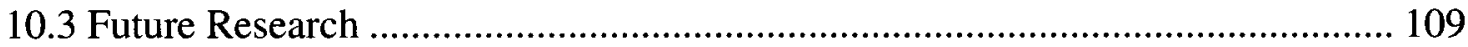

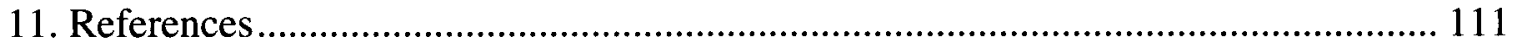

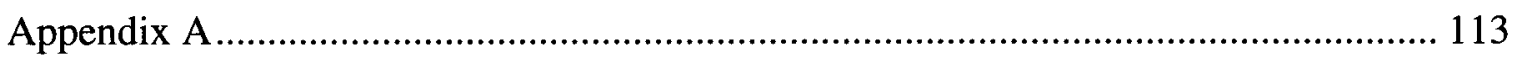

A.1 Pseudocode for Other Cumulative Probability Direction Selection Heuristics ... 113

A.2 Satisfied Votes Branching Direction Heuristic Pseudocode................................ 116

A.3 List of Equality-Containing MIPs................................................................ 117

A.4 List of Equality-Free MIPs ........................................................................... 119

A.5 List of MIPs Where VDS-LCP and METHA Do Not Both Time Out ................. 122

A.6 Constraint and Variable Analysis on the MIP Set ............................................... 126 


\section{List of Tables}

Table 1. Solution counts for the various variable-value pairs ....................................... 11

Table 2. Combinations possible for violated and satisfied constraints ............................. 38

Table 3. Simultaneous variable and direction selection methods using cumulative probabilities......................................................................................................... 44

Table 4. Simultaneous variable and direction selection methods using point probabilities

Table 5. Simultaneous variable and direction selection methods using multiplied probabilities

Table 6. Simultaneous variable and direction selection methods using hybrids ..............48

Table 7. List of existing methods compared against in Experiment 2............................ 52

Table 8. Branching variable direction selection heuristics compared in Experiment 1....55

Table 9. Variable and node selection algorithms used in Experiment 1.........................55

Table 10. Values of mean and variance in a multiple choice constraint........................... 97

Table 11. Values of cumulative probabilities in a multiple choice constraint .................. 97

Table 12. Values of equality ratios in a multiple choice constraint ...................................98 98

Table 13. MIPS that branching up performed poorly on .............................................. 100 


\section{List of Figures}

Figure 1. Pseudocode for finding a feasible solution using B\&B.............................. 6

Figure 2. Graphical representation of a MIP ......................................................... 7

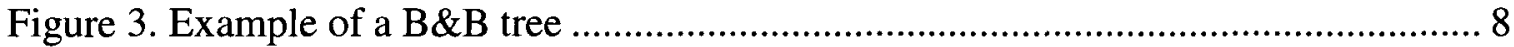

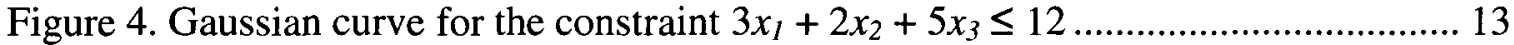

Figure 5. Sample performance profile [Dolan and Moré 2002] ................................. 17

Figure 6. Pseudocode for the Driebeek and Tomlin method [Makhorin 2008] .............. 20

Figure 7. Gaussian curve when branching on $x_{1}$, down direction............................... 32

Figure 8. Gaussian curve when branching on $x_{1}$, up direction.................................. 32

Figure 9. Pseudocode for GetProbabilities function ................................................ 33

Figure 10. Pseudocode for lowest cumulative probability direction selection method .... 37

Figure 11. Pseudocode for violated votes branching direction selection method............ 41

Figure 12. Pseudocode for variable and direction selection using lowest cumulative probability method

Figure 13. Pseudocode for variable and direction selection using lowest point probability

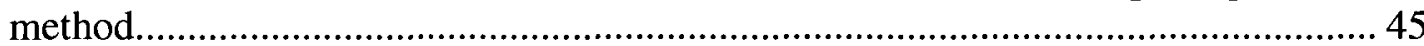

Figure 14. Experiment 1 performance profile for GLPK1 - computation time .............. 56

Figure 15. Experiment 1 performance profile for GLPK1 - number of nodes............... 56

Figure 16. Experiment 1 performance profile for GLPK1 - simplex iterations ............... 57

Figure 17. Experiment 1 performance profile for GLPK2 - computation time ............. 57

Figure 18. Experiment 1 performance profile for GLPK2 - number of nodes................ 58

Figure 19. Experiment 1 performance profile for GLPK2 - simplex iterations.............. 58

Figure 20. Experiment 1 performance profile for GLPK3 - computation time ............. 59

Figure 21. Experiment 1 performance profile for GLPK3 - number of nodes............... 59

Figure 22. Experiment 1 performance profile for GLPK3 - simplex iterations .............. 60

Figure 23. Experiment 1 performance profile for GLPK4 - computation time .............. 61

Figure 24. Experiment 1 performance profile for GLPK4 - number of nodes................ 61

Figure 25. Experiment 1 performance profile for GLPK4 - simplex iterations .............. 62

Figure 26. Experiment 1 performance profile for GLPKDEFAULT - computation time 62

Figure 27. Experiment 1 performance profile for GLPKDEFAULT - number of nodes 63

Figure 28. Experiment 1 performance profile for GLPKDEFAULT - simplex iterations63

Figure 29. Experiment 1 performance profile for METHA - computation time............ 64

Figure 30. Experiment 1 performance profile for METHA - number of nodes .............. 64

Figure 31. Experiment 1 performance profile for METHA - simplex iterations ............ 65

Figure 32. Experiment 1 summary plot for overall data-computation time................66 66

Figure 33. Experiment 1 summary plot for overall data - number of nodes ................. 66

Figure 34. Experiment 1 summary plot for overall data - simplex iterations ............... 67

Figure 35. Experiment 1 summary plot for equality-containing - computation time ..... 67

Figure 36. Experiment 1 summary plot for equality-containing - number of nodes....... 68

Figure 37. Experiment 1 summary plot for equality-containing - simplex iterations ..... 68

Figure 38. Experiment 1 summary plot for equality-free - computation time .............. 69

Figure 39. Experiment 1 summary plot for equality-free - number of nodes ................ 69

Figure 40. Experiment 1 summary plot for equality-free - simplex iterations............... 70 
Figure 41. Experiment 2 performance profile for cumulative and point probability methods on equality-free MIPs - computation time

Figure 42. Experiment 2 performance profile for cumulative and point probability methods on equality-free MIPs - number of nodes...

Figure 43. Experiment 2 performance profile for cumulative and point probability methods on equality-free MIPs - simplex iterations

Figure 44. Experiment 2 performance profile for multiplied probability methods on equality-free MIPs - computation time

Figure 45. Experiment 2 performance profile for multiplied probability methods on equality-free MIPs - number of nodes.

Figure 46. Experiment 2 performance profile for multiplied probability methods on equality-free MIPs - simplex iterations .................................................................... 76

Figure 47. Experiment 2 performance profile for overall data - computation time ......... 77

Figure 48. Experiment 2 performance profile for overall data - number of nodes .......... 78 Figure 49. Experiment 2 performance profile for overall data - simplex iterations......... 78 Figure 50. Experiment 2 performance profile for equality-free MIPs - computation time

Figure 51. Experiment 2 performance profile for equality-free MIPs - number of nodes 80 Figure 52. Experiment 2 performance profile for equality-free MIPs - simplex iterations

Figure 53. Experiment 2 performance profile for equality-containing MIPs - computation time

Figure 54. Experiment 2 performance profile for equality-containing MIPs - number of nodes

Figure 55. Experiment 2 performance profile for equality-containing MIPs - simplex iterations.

Figure 56. Experiment 2 performance profile for HYBRID1 - computation time .......... 83

Figure 57. Experiment 2 performance profile for HYBRID1 - number of nodes............. 84

Figure 58. Experiment 2 performance profile for HYBRID1 - simplex iterations .......... 84

Figure 59. Experiment 2 performance profile for TIES - computation time ................... 85

Figure 60. Experiment 2 performance profile for TIES - number of nodes...................... 86

Figure 61. Experiment 2 performance profile for TIES - simplex iterations .................... 86

Figure 62. Experiment 2 performance profile for VDS-LCP-UP1 - computation time... 87

Figure 63. Experiment 2 performance profile for VDS-LCP-UP1 - number of nodes.... 87 Figure 64. Experiment 2 performance profile for VDS-LCP-UP1 - simplex iterations .. 88 Figure 65. Experiment 2 performance profile for VDS-LCP-UP2 - computation time... 89 Figure 66. Experiment 2 performance profile for VDS-LCP-UP2 - number of nodes.... 89 Figure 67. Experiment 2 performance profile for VDS-LCP-UP2 - simplex iterations .. 90 Figure 68. Experiment 1 summary plots for MIPs with multiple choice constraints-

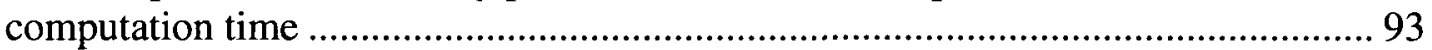

Figure 69. Experiment 1 summary plots for MIPs with multiple choice constraints -

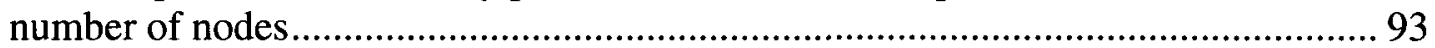

Figure 70. Experiment 1 summary plots for MIPs with multiple choice constraints simplex iterations 
Figure 71. Experiment 1 summary plots for MIPs without multiple choice constraints -

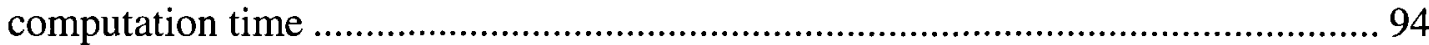

Figure 72. Experiment 1 summary plots for MIPs without multiple choice constraints -

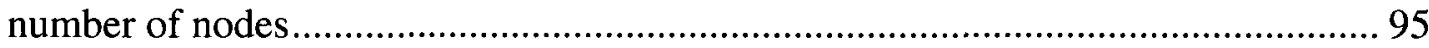

Figure 73. Experiment 1 summary plots for MIPs without multiple choice constraints simplex iterations.

Figure 74. Experiment 2 performance profile for HYBRID2 - computation time ........ 104

Figure 75. Experiment 2 performance profile for HYBRID2 - number of nodes.......... 105

Figure 76. Experiment 2 performance profile for HYBRID2 - simplex iterations ........ 105 


\section{Introduction}

A Mixed Integer Programming (MIP) problem is a linear program in which some or all of the variables are required to take on integer or binary values. [Winston 2004]. MIPs are composed of an objective function, general constraints, integrality restrictions and variable bounds. In general a MIP looks like:

$$
\begin{aligned}
& \text { Objective: } \quad \operatorname{Max} Z=c x \\
& \text { General constraints: } \quad \boldsymbol{A x}\{\leq, \geq,=\} \boldsymbol{b} \\
& \text { Integrality restrictions: } \quad x_{1}, x_{2}, \ldots, x_{p} \text { are integer variables; } x_{p+1}, x_{p+2}, \ldots \text {, } \\
& x_{n} \text { are real variables. } \\
& \text { Variable bounds: } \quad l \leq x \leq u,
\end{aligned}
$$
problems.

The above description of a MIP assumes that the problem is a maximization problem. Converting this model to represent a minimization problem is achieved by multiplying the objective function by -1 .

MIPs can be used to model a wide range of real-world applications. Some examples include but are not limited to evaluating investment options, supply chain optimization, airline scheduling, production planning, and packing (or knapsack) type problems [Winston 2004]. 
Branch and Bound (B\&B) is a common method used to solve MIPs. It works by performing a relaxation of the integer constraints, also called a Linear Program (LP) relaxation, and solving the resulting $\mathrm{LP}$. The $\mathrm{B} \& \mathrm{~B}$ solution is generated by a tree structure, reflecting the various branching and bounding decisions made. This method will be described in more detail in Section 2.1. There are three important decisions made in designing a $\mathrm{B} \& \mathrm{~B}$ algorithm: the node selection algorithm, the branching variable selection algorithm and the branching variable direction selection algorithm. A variety of heuristics can be used for each. Some of these heuristics will be highlighted in Sections 2 and 3.

There are two options that the branching variable direction selection algorithm must choose between: branching up and branching down. The meaning of branching up and branching down will be explained in Section 2. Heuristics for selecting the branching variable direction is not a heavily researched topic in MIP literature. In fact, there have been no empirical studies on which branching variable direction heuristic is best. However, folklore tends to say that branching up at every node is generally the best policy. The focus of this thesis is to identify the best branching variable direction selection heuristic.

Experiments are conducted by comparing the branching up always policy to other existing branching direction algorithms as well as several new branching variable direction algorithms. The branching variable direction selection heuristics are also extended to select both the branching variable and the branching direction later in the thesis. These branching variable selection methods are compared to the state of the art in variable selection, and their relative merits are discussed. 
All experiments in this document are conducted in order to find the first integerfeasible solution to the MIP rather than an optimal solution. These experiments demonstrate that branching up always is the best policy when solving MIPs to first integer feasibility. They also result in the first empirical study of branching variable direction selection algorithms. In addition, a new variable selection and branching direction selection algorithm is shown to perform equal to the state of the art in reaching first integer feasibility quickly. 


\section{Background}

\subsection{Using the Branch and Bound Method to Solve MIPs}

As stated in Section 1, a MIP is a linear program in which some or all of the variables are required to take on integer or binary values. Integrality constraints and variable bounds work together to restrict the values of integer variables to either general integer values $(\ldots,-1,0,1,2, \ldots)$ or binary values $(0$ or 1$)$. A feasible solution to the MIP is a solution that satisfies all of the constraints and integrality restrictions; whereas the LP feasible solution satisfies all of the general constraints, but not necessarily all of the integrality restrictions. If all of the variables in the MIP are integer variables, then the problem is called Integer Programming (IP). IPs are special cases of MIPs. Binary Integer Programming (BIP) problems are special cases of IPs where all of the variables must have binary values. The term MIP will be used to describe MIPs, IPs, and BIPs in this document.

The B\&B method is the main algorithm used to solve MIPs. In this method, the integer constraints are initially relaxed, and the resulting LP is solved. At this LP relaxation some of the integer variables may be assigned integer values, and some may not. An integer variable that does not have an integer value in the LP relaxation solution is called a candidate variable. The $\mathrm{B} \& \mathrm{~B}$ algorithm next selects one of the candidate variables as the branching variable. The branching creates two child nodes for which additional bounds are placed on the LP relaxation. The bounds ensure that the candidate variable cannot have its current non-integer value henceforth. Branching in the up 
direction (branching up) means a lower bounding constraint is placed on the candidate variable as follows:

$$
\text { CandidateVariable } \geq\lceil\text { CurrentValue }\rceil .
$$

Whereas branching in the down direction (branching down) means an upper bounding constraint is placed on the candidate variable as follows:

$$
\text { CandidateVariable } \leq\lfloor\text { CurrentValue }\rfloor .
$$

A tree structure is generated by exploring various combinations of the variable settings. Each variable combination is called a node in the tree [Patel and Chinneck, 2007]. Exploring all possible variable-value combinations is time consuming and not feasible for medium to large-sized MIPs, so there are many algorithms and heuristics to select which variable to branch on, which direction to branch in (up or down), as well as which node in the tree to explore next. To date there is no defined "best method" to find a feasible or optimal solution to MIPs in the most efficient manner. As the focus of this thesis is finding first-feasibility only, the process for using B\&B to find a feasible solution in a MIP is outlined in the pseudocode in Figure 1. 
Input: MIP.

0 . Initialize: List of unexplored nodes $=\phi$.

1. The root node is the original MIP model. Solve the LP relaxation of the root node.

1.1 If LP relaxation is LP-infeasible, exit with infeasible outcome.

1.2 If LP relaxation is integer-feasible, exit with LP relaxation as solution.

2. Select a candidate variable from the current node for branching.

3. Create the two child nodes from the current node by adding bounds about the candidate variable.

3.1 ChildNode1 has the added constraint CandidateVariable $\geq\lceil$ CurrentValue $\rceil$

3.2 ChildNode2 has the added constraint CandidateVariable $\leq\lfloor$ CurrentValue

4. Add these nodes to the list of unexplored nodes.

5. Store the preference for which of the two child nodes to expand next (up or down direction).

6. Select a node from the list of unexplored nodes based on the node selection algorithm and the child node preference in Step 5.

6.1 If there are no unexplored nodes, exit with infeasible outcome.

7. Solve the LP relaxation of the selected node.

7.1 If LP relaxation is infeasible, discard node and go to Step 6.

7.2 If LP relaxation is integer-feasible, exit with LP relaxation as solution.

8. Go to Step 2.

Figure 1. Pseudocode for finding a feasible solution using B\&B

There are three situations, when using the B\&B method to solve a MIP, that a decision is made. One situation is selecting the next variable to branch on. The second situation is then deciding which direction to branch in, either branching up or down to one of the child nodes. The third decision is which node to expand from the list of unexplored nodes. All three decisions have an effect on the speed at which a solution to the MIP is obtained. In a perfect world, there would be an algorithm that would always choose the "correct" next variable to branch on, the "correct" direction, as well as the "correct" node to solve the LP relaxation for. This would result in one branch of the tree being explored and it would lead directly to a feasible solution. Of course, this magical algorithm does not currently exist. The act of exploring one branch in a tree, while 
continually going deeper in the tree, is called a dive. Each time the algorithm back-tracks up to a different branch of the tree, another dive is accumulated.

Figure 3 and the following discussion expand upon the importance of the three $\mathrm{B} \& \mathrm{~B}$ decisions. For this example the following MIP is used:

$$
\begin{aligned}
& \text { Max } Z=x+3 y \\
& -3 x+5 y \leq 8 \\
& 5 x+4 y \leq 39 \\
& x, y \text { are integer variables } \\
& x, y \geq 0 .
\end{aligned}
$$

Figure 2 below graphically displays the above example. The solid lines represent the constraints, the dotted line represents the objective function at a value of ten, and the dots represent all of the integer feasible points within the LP relaxation's feasible region.

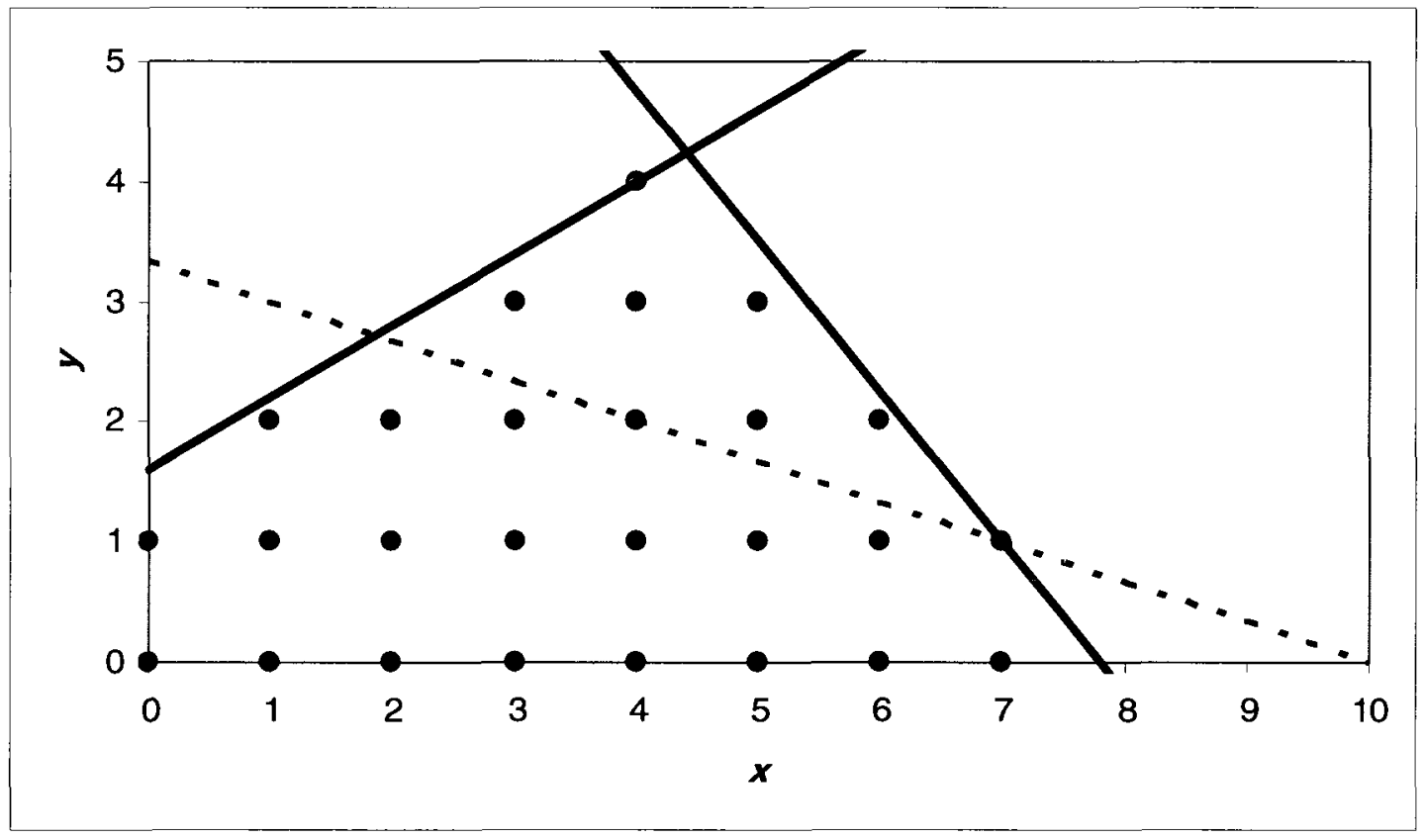

Figure 2. Graphical representation of a MIP 
In Figure 3, each node in the tree is represented by a square, and is numbered according to the order it was solved in. Each square shows the LP-relaxation values for $Z$, $x$, and $y$, as well as whether the solution is feasible or infeasible. The lines connecting the nodes are labelled with the bounding constraint added in Step 3 of the algorithm. The tree is expanded past the first feasible solution to demonstrate other paths in the tree that lead to feasible solutions. These additional feasible solutions are shown in dashed boxes.

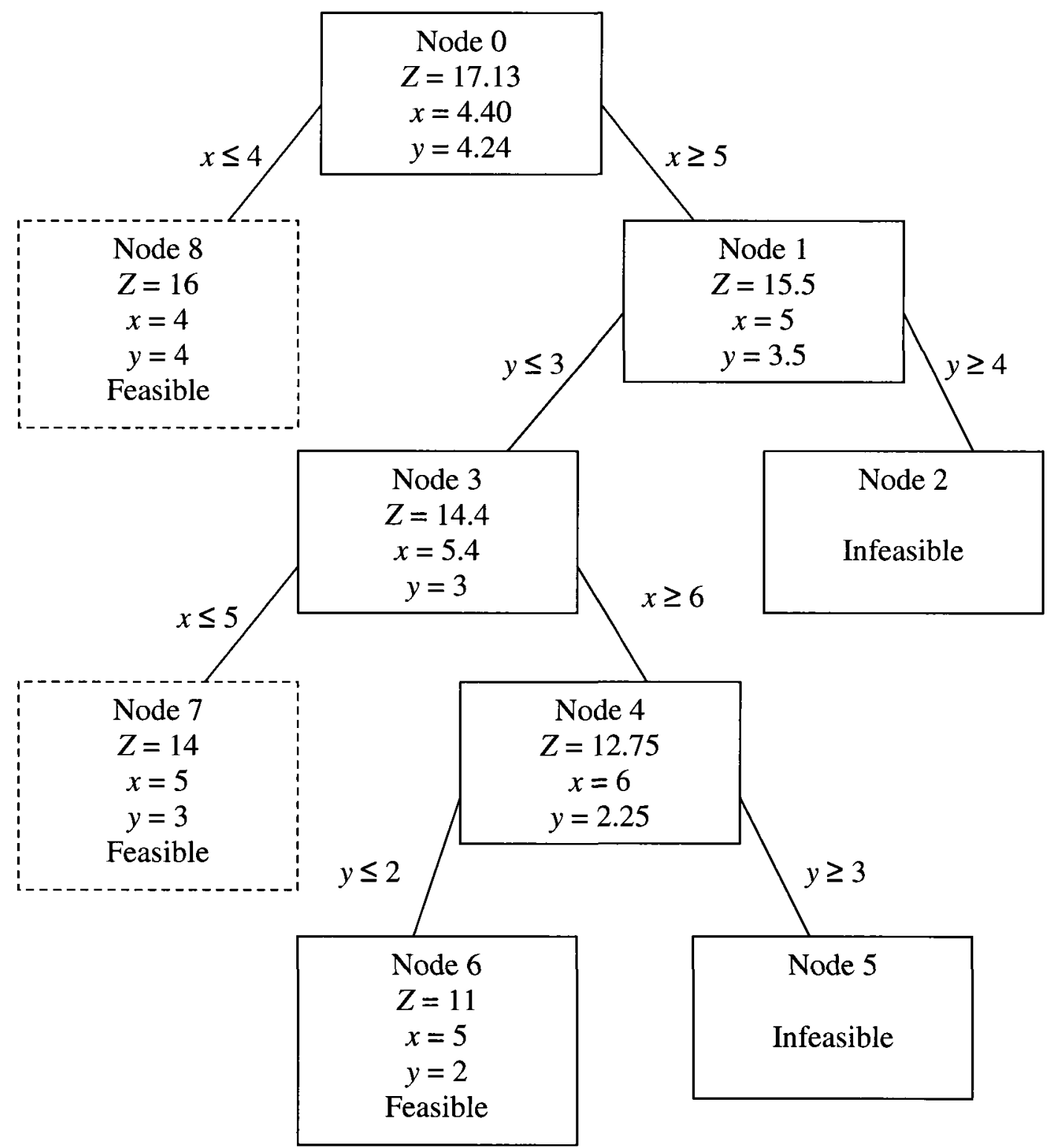

Figure 3. Example of a $B \& B$ tree 
Using Figure 3 as an example, the ideal path to find a feasible solution quickly would be to expand Node 0 , followed by Node 8 . This path consists of one dive in the tree, and results in an immediate feasible solution. However, the solution in Figure 3 expands Node 1 first, which requires several branches before finding a first feasible solution. The decision to expand Node 1 before Node 8 is a branching direction decision. Ideally in this instance, the desired algorithm would select branching down at Node 0 , rather than branching up. An example of a poor node selection decision occurs at Node 2 . Node 2 is infeasible, so the algorithm discards it and selects Node 3 as the next node to expand. However, if Node 8 were selected for expansion (both Node 3 and 8 are present in the unexplored nodes list), a feasible solution would have been found more quickly. Choosing Node 8 for expansion leads to a feasible solution in one further node expansion; whereas following Node 3 requires four more node expansions before a feasible solution is reached.

The third $\mathrm{B} \& \mathrm{~B}$ decision is branching variable selection. In the above example, the first branching variable selected is $x$. The B\&B tree would look completely different if $y$ had been selected as the first branching variable. Choosing $y$ would make the first up node infeasible, and the down node a feasible solution $(x=4, y=4, Z=16)$. This means that whichever direction the algorithm chooses to branch in, it will arrive at a feasible solution in at most two node expansions. This is a far smaller B\&B tree than Figure 3. As demonstrated, selecting the branching direction, selecting the branching variable, and selecting the next node for expansion are three important decisions that have large effects on the size of the resulting $\mathrm{B} \& \mathrm{~B}$ tree. Making branching variable selection and node selection decisions intelligently is important to the speed and resources required to solve 
the MIP [Achterberg et al. 2005, Ching-Jong 1994, Linderoth et al. 1999, Linderoth et al. 2004, Patel and Chinneck 2007, Winston 2004]. This thesis shows that the algorithm used to select the branching direction also has an effect on the speed and resources required to solve the MIP.

\subsection{Counting Solutions of Knapsack Constraints}

Gilles Pesant and Claude-Guy Quimper [Pesant and Quimper 2008] discuss effective heuristics that count solutions to approximate the tightness of a constraint (specifically integer knapsack constraints in their case). An integer knapsack constraint is defined as:

$$
l \leq c x \leq u,
$$

where $\boldsymbol{c}$ is an integer row vector, $\boldsymbol{x}$ is a column vector of finite variables $\left(x_{1}, x_{2}, \ldots, x_{n}\right)$, and $l$ and $u$ are integers. In addition, all integers are non-negative. For these types of constraints, Peasant and Quimper suggest counting the number of variable-value pairs that will satisfy the constraint for those variables not yet fixed. The solution count is then used as a measure of how tight the constraint is, or how much emphasis should be placed on the constraint.

The Pesant and Quimper heuristic can be illustrated by a simple example. Take the following constraint:

$$
x_{1}+5 x_{2} \leq 10,
$$

where both $x_{1}$ and $x_{2}$ are candidate variables. Each variable-value pair has a solution count for the constraint, or the number of solutions that satisfy the constraint. In other words, a solution count can be generated when one of the candidate variables is temporarily assigned a value. Then the remaining variables in the constraint can vary 
their values and each allowable combination counts as one solution. The solution counts for the above constraint are illustrated in Table 1 below. For example, if $x_{2}$ equals $0, x_{1}$ can take any value between 0 and 10 while satisfying the constraint, so the solution count for the fixed variable-value pair of $x_{2}$ equals 0 , is 11 .

Table 1. Solution counts for the various variable-value pairs

\begin{tabular}{|c|c|c|c|}
\hline Variable & Variable Value & Allowable Range for Remaining Variable & Solution Count \\
\hline$x_{2}$ & 0 & {$[0,10]$} & 11 \\
\hline$x_{2}$ & 1 & {$[0,5]$} & 6 \\
\hline$x_{2}$ & 2 & {$[0]$} & 1 \\
\hline$x_{1}$ & 0 & {$[0,2]$} & 3 \\
\hline$x_{I}$ & 1 & {$[0,1]$} & 2 \\
\hline$x_{I}$ & 2 & {$[0,1]$} & 2 \\
\hline$x_{I}$ & 3 & {$[0,1]$} & 2 \\
\hline$x_{1}$ & 4 & {$[0,1]$} & 2 \\
\hline$x_{1}$ & 5 & {$[0,1]$} & 2 \\
\hline$x_{1}$ & 6 & {$[0]$} & 1 \\
\hline$x_{I}$ & 7 & {$[0]$} & 1 \\
\hline$x_{1}$ & 8 & {$[0]$} & 1 \\
\hline$x_{I}$ & 9 & {$[0]$} & 1 \\
\hline$x_{1}$ & 10 & {$[0]$} & 1 \\
\hline
\end{tabular}

Clearly, from Table 1, the variable-value assignment that provides the most future options for satisfying the constraint is when $x_{2}$ is forced to 0 . It is also interesting to note that there are several variable-value assignments that provide the smallest number of future options for satisfying the constraint. These variable-value assignments show the tightness of the constraint, or how difficult it will be to solve, if it is not satisfied immediately.

From the solution count, a solution density can be calculated as follows:

$$
\frac{\# \text { Counts }_{x_{i}=d}}{\# \text { Counts }} \text { constraint } \text {, }
$$


where $x_{i}$ is the variable whose solution density is being calculated. The value of $x_{i}$ is $d$. The numerator can be read as the number of solution counts for one constraint when $x_{i}$ equals $d$. The denominator can be read as the number of solution counts for any variablevalue combination, on that one constraint (referenced in the numerator). Solution density measures how often a certain assignment is part of a feasible solution. From the above example the solution density when $x_{2}$ equals 0 is calculated as follows:

$$
\text { SolutionDensity } x_{x_{2}=0}=\frac{11}{11+6+1+3+2+2+2+2+2+1+1+1+1+1}=0.3056 .
$$

Therefore $30.56 \%$ of all feasible variable-value combinations for this constraint occur when $x_{2}$ is assigned the value of 0 .

The solution densities can then be applied to generate a Gaussian curve when the variables themselves have lower and upper bounds. To take a step back, this occurs because each candidate variable is assumed to be equally likely to be assigned any of the values in its allowable range. This represents a discrete uniform distribution. When looking at all of the variables in a constraint, the distribution needed is a linear combination of uniformly distributed random variables. The linear combination approximates a continuous normal distribution using the central limit theorem. This normal distribution has a mean and variance as follows:

$$
\begin{gathered}
\mu=\sum_{i=1}^{n} c_{i} \frac{a_{i}+b_{i}}{2}, \\
\sigma^{2}=\sum_{i=1}^{n} c_{i}^{2} \frac{\left(b_{i}-a_{i}+1\right)^{2}-1}{12},
\end{gathered}
$$

where there are $n$ variables $\left(x_{1}, x_{2}, \ldots, x_{n}\right)$ in a constraint. Each variable, $x_{i}$, has an allowable range $\left[a_{i}, b_{i}\right]$, and a coefficient, $c_{i}$ in the constraint.

Using the following constraint as an example, 


$$
3 x_{1}+2 x_{2}+5 x_{3} \leq 12
$$

with all variables integer and restricted to values between $[0,5]$, results in the normal distribution given in Figure 4. Figure 4 shows that the mean of this distribution is 25 and the variance is 110.83 . This curve now shows the probability of the various variablevalue combinations having solutions. The $x$-axis in the graph shows the range of possible right-hand-side (RHS) values that the constraint can have. Since the RHS is known to be 12 in this case, the cumulative probability of achieving this value given the allowable ranges of the variables is the area under the curve from $x$ equals 0 to 12 . This curve provides a way to calculate the likelihood of satisfying the constraint.

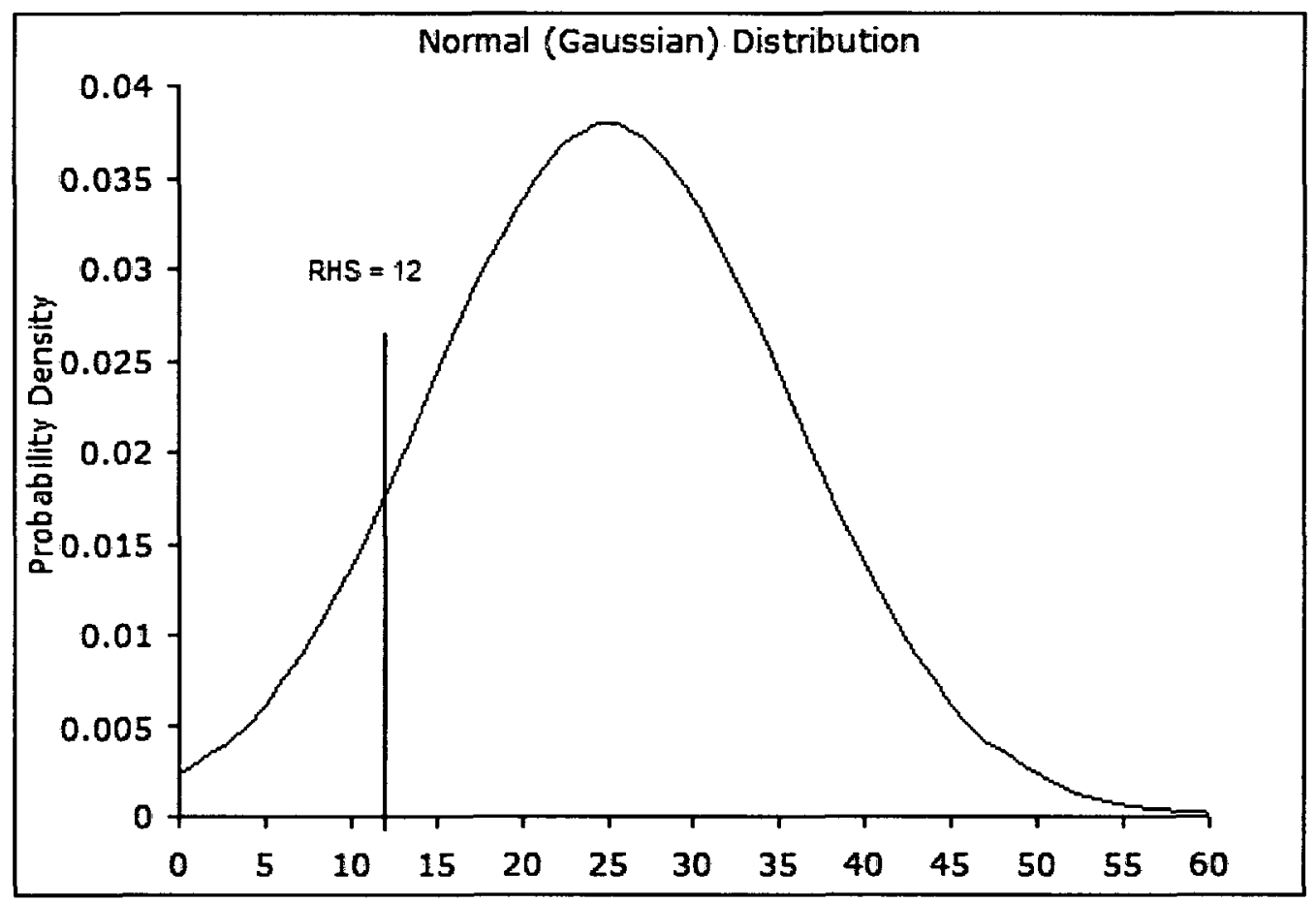

Figure 4. Gaussian curve for the constraint $3 x_{1}+2 x_{2}+5 x_{3} \leq 12$

Unfortunately, the normal cumulative distribution function cannot be integrated analytically to determine the area under the curve [Brooks 1998]. In order to calculate the cumulative probability itself, an approximation is required. One of the more common 
approximations used today is algorithm 26.2.17 from Abromowitz and Stegun [1965] which has a maximum absolute error of $7.5 \times 10^{-8}$. The algorithm and a sample calculation using the constraint in Figure 4 is shown below:

$$
\begin{gathered}
P(x)=1-Z(x) \times\left(b_{1} t+b_{2} t^{2}+b_{3} t^{3}+b_{4} t^{4}+b_{5} t^{5}\right) \text { when } x \geq 0 . \\
P(x)=Z(x) \times\left(b_{1} t+b_{2} t^{2}+b_{3} t^{3}+b_{4} t^{4}+b_{5} t^{5}\right) \text { when } x<0 .
\end{gathered}
$$

$P(x)$ is the cumulative probability approximation function, where:

$$
\begin{gathered}
Z(x)=c \times e^{-x^{2} / 2}, \\
t=\frac{1}{1+p x} \text { when } x \geq 0 \text {, and } t=\frac{1}{1-p x} \text { when } x<0 \\
x=\frac{R H S-\mu}{\sigma},
\end{gathered}
$$

and $R H S$ is the right-hand side of the constraint the probability is being calculated for.

There are several constants in the above formulae with values as follows:

$$
\begin{gathered}
b_{1}=0.319381530, b_{2}=-0.356563782, b_{3}=1.781477937, b_{4}=-1.821255978, \\
b_{5}=1.330274429, p=0.2316419, c=0.39894228 .
\end{gathered}
$$

Using the above $3 x_{1}+2 x_{2}+5 x_{3} \leq 12$ example, $\mu=25, \sigma^{2}=110.83$, and $R H S=12$.

Therefore:

$$
\begin{gathered}
x=\frac{12-25}{\sqrt{110.83}}=-1.23485, \\
t=\frac{1}{1-(0.2316419)(-1.23485)}=0.777579, \\
Z(-1.23485)=0.39894228 \times e^{-(-1.23485)^{2} / 2}=0.186119,
\end{gathered}
$$

and 


$$
\begin{gathered}
P(x)=0.186119 \times\left(0.319381530 \times 0.777579+(-0.356563782) \times 0.777579^{2}\right. \\
\left.+1.781477937 \times 0.777579^{3}+(-1.821255978) \times 0.777579^{4}+1.330274429 \times 0.777579^{5}\right) \\
\text { or } P(x)=0.108443 .
\end{gathered}
$$

In other words, the cumulative probability that the constraint $3 x_{1}+2 x_{2}+5 x_{3} \leq 12$ can be satisfied given that $x_{1}, x_{2}$, and $x_{3}$ are restricted to integer values between $[0,5]$ is 0.1084 .

Section 5 will describe how the constraint function value probability can be used to select a branching variable and a branching variable direction.

\subsection{Performance Profiles}

Benchmarking algorithms effectively is an important aspect of empirical analysis. Performance profiles have emerged as an accepted method to compare algorithms on various performance metrics [Dolan and Moré 2002].

Typical benchmarking techniques involve tables of data that display the results of each algorithm with respect to a metric such as computing time required, number of iterations or number of function evaluations. However, these tables can be difficult to sift through due to their size if the test set is large. Several tools were developed to analyze the tables. When using averages or cumulative metrics, oftentimes the results are dominated by a small number of difficult problems. Averaging also biases the results against an algorithm that may be more robust than another, simply because any failed attempts are discarded and not penalized. In this thesis, when an algorithm is said to be "more robust" than another algorithm, that means that the algorithm is able to solve/complete more of the problem set in the designated time limit. Ranking the methods, by counting the number of times a method comes in $k^{\text {th }}$ place, loses the size of 
the improvement of one method over another. By comparing medians or quartiles of a metric, information about trends is lost, and there are often big jumps in improvement rather than gradual improvements in algorithms. Clearly there are flaws with all of these techniques.

The solution to these problems is performance profiles. Performance profiles generate a cumulative distribution function for each performance metric. For example if computing time is the metric, values in the performance profile tables are the ratio of the computing time a method took to solve a problem, over the best time any of the methods had in solving that same problem. This is also stated as:

$$
r_{p, m}=\frac{t_{p, m}}{\min \left(t_{p, m}: m \in M\right)}
$$

where $r$ is the performance ratio of a particular method, $m$, on problem, $p, t_{p, m}$ is the time required for that method to solve that problem, and $M$ is the set of all methods being compared.

The above formula is used on a case by case basis to create the cumulative distribution, which is formulated below:

$$
\rho_{m}(\tau)=\frac{1}{n_{p}}\left|p \in P: r_{p, m} \leq \tau\right|
$$

where $\rho_{m}(\tau)$ is the probability for method $m$, that $r_{p, m}$ is within a factor of $\tau$ of the best possible ratio. $n_{p}$ is the number of problems in the test set. The function $\rho_{m}$ is the cumulative distribution function for the performance ratio. For example, $\rho_{m}(l)$ is the probability that method $m$ will win or tie for best value over all the other methods. Ideally the goal is to find a method that will always win. An example of a performance profile is shown below in Figure 5. 


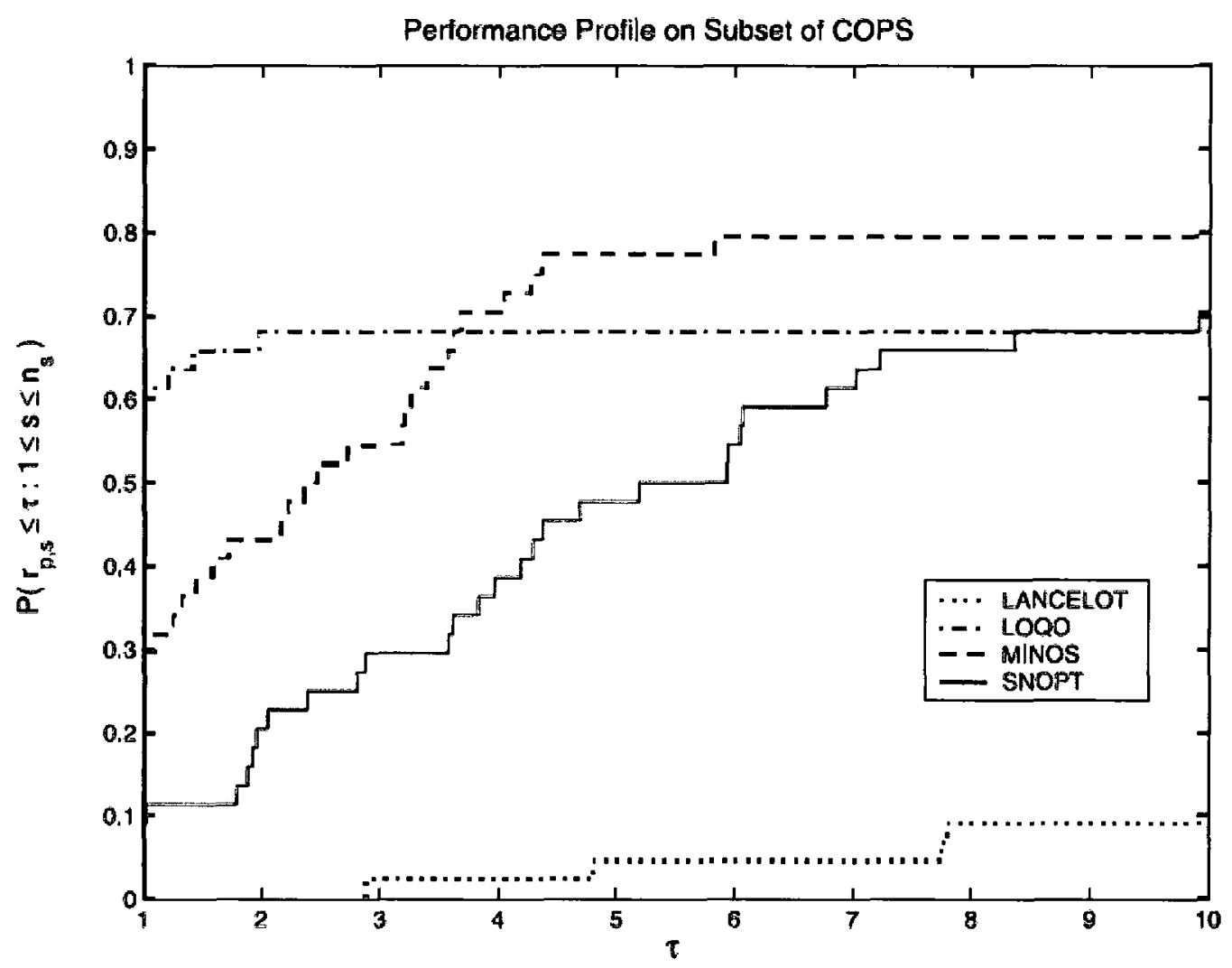

Figure 5. Sample performance profile [Dolan and Moré 2002]

In this performance profile, four solvers are compared by their computing time on a set of problems. The LOQO solver has the most wins, solving $60 \%$ of the problem set within the ratio to best $(\tau)$ of one. However, as the performance profile progresses, the improvement in the MINOS solver is clear, as it overtakes LOQO as the best solver after a ratio to best of four. This means that MINOS is within a factor of four to the best solver more often than LOQO. In other words, MINOS may not always be the best, but it is close to the best most of the time. As the MINOS line continues upward, it stops around the $80 \%$ mark. This means that MINOS was the solver able to solve the most problems in the problem set; it is the most robust solver tested. LOQO on the other hand, can only solve $70 \%$ of the problem set, and so although it has the most wins, it also has the 
disadvantage that it solves a smaller variety of problems. In this manner, performance profiles provide a graphical story about the relative merits and disadvantages of the methods being compared, rather than a simple table of numbers.

Performance profiles are used to evaluate the algorithms for four reasons. Firstly, performance profiles eliminate the influence of a small number of problems because cumulative probabilities are used rather than averages. Performance profiles also eliminate the sensitivity associated with ranking solvers, as the ranking or ratio to best, is shown over a continuous scale. In addition, they provide the analyst with a visual representation of the expected performance difference amongst different methods, which is easier to understand than a large table of numbers. Finally, performance profiles avoid the problems with discarding failed trials because the robustness of a method is evident in the graph itself.

\subsection{Branching Variable Selection Algorithms}

This section discusses in depth several of the branching variable selection algorithms used for comparison purposes in Sections 7 and 8. The algorithms explained in this section are: most fractional branching, first fractional branching, last fractional branching, the Driebeek and Tomlin algorithm, and active-constraint variable selection. The most fractional branching (GLPK1) or most infeasible branching rule [Achterberg et al 2005] simply branches on the integer variable whose fractional part is currently closest to 0.5 . The logic behind the rule is that this variable is the most difficult to decide whether to branch up or down to the next integer (in order to be feasible). So by 
branching on it, the variable is no longer an issue as the new variable bounds at its child nodes will remove this particular decision.

Two other branching variable selection methods are first fractional (GLPK2) and last fractional (GLPK3) branching selection rules [Achterberg et al 2005]. In these two methods, the algorithm looks at the list of candidate variables, and selects the first (or last) candidate variable in the list as the branching variable. It should be noted that none of the three above branching variable selection methods specify which branching direction is selected.

Driebeek developed a well-known branching variable selection algorithm that also selects the branching variable direction [Driebeek 1966]. Tomlin extended Driebeek's idea by considering the integrality of variables [Tomlin 1971]. In this thesis, the improved algorithm is referred to as the Driebeek and Tomlin (GLPK4) method. The Driebeek and Tomlin method is a penalty method that estimates the potential degradation of the objective function value when selecting a candidate variable. The degradation is estimated by performing a dual simplex pivot which generates a lower bound on the bound improvement possible if a given candidate variable was selected as the branching variable [Linderoth and Ralphs 2004]. The pseudocode for the Driebeek and Tomlin method is shown below in Figure 6. In this pseudocode, DeltaZ is the estimation of the degradation of the objective function value. 
Input: MIP.

0 . Initialize: degradation $\leftarrow-1 ;$ Delta $Z \leftarrow 0$

1. For each candidate variable, called candidate:

1.1 primal $\leftarrow \mathrm{LP}$ relaxation optimum value of candidate

1.2 Compute candidate's corresponding row in the simplex table

1.3 For the up branching direction and the down branching direction:

1.3.1 Perform the dual simplex pivot to determine which variable, $k$, should enter the basis to keep dual feasibility

1.3.2 alpha $\leftarrow$ Coefficient value of variable $k$ in the current row 1.3.3 If the branching direction is $u p$ :

1.3.3.1 DeltaX $\leftarrow\lfloor$ primal $\rfloor-p r i m a l$

1.3.4 Else: DeltaX $\leftarrow\lceil$ primal $\rceil$ - primal

1.3.5 DeltaK $\leftarrow$ DeltaX / alpha

1.3.6 If $k$ is an integer variable:

1.3.6.1 If DeltaK $\geq 0$ :

1.3.5.1.1 DeltaK $\leftarrow\lceil$ DeltaK $\rceil$

1.3.6.2 Else: DeltaK $\leftarrow\lfloor$ DeltaK $\rfloor$

1.3.7 $d k \leftarrow$ Reduced cost of $k$ in the current basis

1.3.8 DeltaZ $\leftarrow d k \times$ DeltaK

1.3.9 If the branching direction is $u p$ :

1.3.9.1 DzUp $\leftarrow$ DeltaZ

1.3.10 Else: $D z D n \leftarrow$ DeltaZ

1.4 If degradation < abs $(D z D n)$ or degradation $<\operatorname{abs}\left(D_{z} U p\right)$ :

1.4.1 Branching variable selected for branching is candidate

1.4.2 If $\operatorname{abs}(D z D n)<\operatorname{abs}\left(D_{z} U p\right)$ :

1.4.2.1 branching variable direction selection is in down direction

1.4.2.2 degradation $\leftarrow D z U p$

\subsubsection{Else:}

1.4.3.1 branching variable direction selection is in up direction 1.4.3.2 degradation $\leftarrow D z D n$

Figure 6. Pseudocode for the Driebeek and Tomlin method [Makhorin 2008]

To summarize the algorithm, Tomlin's addition is step 1.3.6, where integrality is considered. The algorithm looks at each of the candidate variables, and performs a dual simplex pivot to determine which variable would enter the basis if the current candidate left the basis. Based upon this change to the basis, the degradation of the objective function value can be calculated. Two degradation values are calculated: one for the 
branching up decision, one for the branching down decision. Degradation-up and -down are compared to the current largest degradation (from looking at the other candidate variables). If either is larger than the current degradation, then the associated candidate variable is selected as the branching variable. It is assigned the branching direction of smallest degradation. The current best degradation is updated as the larger degradation of the -up and -down values.

The four algorithms above focused on selecting the candidate variable with the greatest impact on the objective function. However, the point of active-constraint variable selection [Patel and Chinneck 2007] is to branch on the candidate variable that has the greatest impact on the active constraints in the LP relaxation at the current node. This method was developed and tested in an environment to find the first feasible solution quickly. The active constraints are the constraints in the LP relaxation that are determining the LP solution (if one of the active constraints was changed, the LP solution would change, unless there was degeneracy). One may want to branch on the variable with the most impact on the active constraints because the child nodes will exhibit the largest difference between their respective bounding function values. The two solutions would be the farthest apart. This would hopefully mean that one child node has a good solution, and the other child node does not and will never have to be expanded. It reduces the search tree significantly if used early in the branch and bound process.

The basic algorithm for the active-constraint variable selection method consists of three steps. First, normalize the active constraints. Then, assign a weight to each candidate variable (based upon its impact on the active constraints). Finally, the candidate variable with the highest total weight is selected as the branching variable [Patel and 
Chinneck 2007]. There are several different ways to estimate the impact of the candidate variables on the active constraints. There are basically two categories of estimates: how much impact a candidate variable has within one active constraint, and how much the complete set of active constraints can be influenced by one variable.

One of the many active-constraint variable selection methods is called Method A (METHA). Method A simply counts the number of occurrences a candidate variable has in all of the active constraints. The candidate variable with the most appearances in the active constraints is then selected for branching [Patel and Chinneck 2007].

The active-constraint variable selection method is used to find the first feasible solution to the MIP. When compared with CPLEX 9.0 with its internal heuristics turned off the active-constraint variable selection methods tend to outperform it in terms of number of nodes visited and number of simplex iterations performed. Overall, the method shows promise for reducing the time required to find the first feasible solution in a MIP [Patel and Chinneck 2007]. Due to the fact that Method A was among the best of the active-constraint variable selection methods and tested well against CPLEX 9.0, it was selected as a comparison algorithm for the experiments in Sections 7 and 8 to represent the active-constraint variable selection methods as a whole.

\subsection{Node Selection Algorithms}

The most common node selection scheme for solving MIPs using the branch and bound method is known as depth first [Patel and Chinneck 2007]. Strict Depth first expands the node with the best bounding function at the maximum depth level in the branch and bound tree. In other variants of depth first node selection, when an infeasible node is 
found, a different node selection scheme is triggered to decide which node to jump back to. Depth first is then continued after the jump back. This thesis deals only with strict depth first node selection. This type of node selection policy tends to minimize the memory requirements of the computer program because the parent and child nodes are so similar. In addition, it only has to make minor changes in the LP relaxation from one node to the next (usually just one variable bound is added to the LP). Also because it searches so deep in the tree, it tends to find feasible solutions faster than other node selection techniques [Linderoth and Savelsbergh 1999]. In particular, Patel and Chinneck [2007] concluded that when the purpose of the experiment is achieving integer feasibility quickly (as is the case in this thesis), depth first search is preferred.

Best local bound or locally best bound is a node selection policy that selects the next node for expansion based upon the best bounding function value of a subset of the unsolved nodes list. This strategy minimizes the total number of nodes stored in the core storage of the solver so that it is only selecting the next branching node from a subset of the total available nodes [Ching-Jong 1994]. When the number of solved nodes reaches a predetermined number, the nodes are ordered according to their bounding function, and the nodes with the better bounding functions are kept in the core memory. The rest of the nodes are stored in auxiliary storage and are called node sets. Now one can only select a node for expansion from the nodes stored in the core memory. When there are no nodes left in the core memory, a node set from the auxiliary storage is brought into the core memory and is expanded. This process continues until there are no node sets left in the auxiliary storage [Ching-Jong 1994]. 
Best local bound performs well as a method. However the benefit of using it is most profound when the computation time in each node is relatively small, and therefore it is most likely not a good strategy for an LP based B\&B method when compared to depth first node selection [Ching-Jong 1994]. For this reason, best local bound was only used as the node selection method for the tests comparing against the default settings in the MIP solving software. 


\section{State of the Art in Branching Variable Direction Selection}

Very few studies of MIP solution algorithms mention the branching variable direction selection heuristic used, let alone compare various branching direction methods. This section discusses the few instances in the literature in which a branching variable direction heuristic is mentioned.

\subsection{Simple Branching Variable Direction Heuristics}

First of all, there are a few simple branching variable direction selection heuristics in place to date. These methods are: branch up, branch down, and closest integer.

Branch up (UP) simply means that each time a candidate variable is selected for branching, a lower bound is added to the LP ensuring the candidate variable takes on a value at least as large as its current primal value rounded up to the next integer [Patel and Chinneck 2007, Makhorin 2008]. In other words the constraint added is:

$$
\text { CandidateVariable } \geq\lceil\text { CurrentValue }\rceil .
$$

Branch down (DOWN) is simply the opposite of branch up [Patel and Chinneck 2007, Makhorin 2008]. An upper bound is added:

$$
\text { CandidateVariable } \leq\lfloor\text { CurrentValue }\rfloor .
$$

Closest integer $(\mathrm{CI})$ means the primal value of the branching variable is rounded to the nearest integer. If the value rounds up, the branch up bound is added to the LP, if the value rounds down, the branch down bound is added to the LP [Patel and Chinneck 2007, Makhorin 2008]. It is also possible, although not mentioned in the literature, to branch in the direction farthest from integrality. 


\subsection{Studies Discussing Branching Variable Direction Selection}

As mentioned before, very few papers discuss branching variable directions. Patel and Chinneck [2007] even state that branch up is selected arbitrarily as the branching variable direction heuristic for the active-constraint variable selection methods. Driebeek and Tomlin [1966, 1971] use their built-in heuristic for selecting the branching variable direction as mentioned in Section 2.4, but it is never compared to the other basic heuristics to justify its usage. Makhorin [2008] uses closest integer as the branching direction heuristic in the source code for GLPK, but makes no reference to this fact in the reference manual.

Meyer et al. [2003] discusses branching variable direction selection during their application of B\&B strategies to Brachytherapy treatment optimization. They try various combinations of $\mathrm{B} \& \mathrm{~B}$ techniques to find the optimal placement of the radioactive seeds for the cancer treatment. In their MIP formulation, each binary variable corresponds to the placement or non-placement of a seed in a particular location. The placement of seeds is limited by the total allowable dosage. Its goal is to deliver a dose to the cancerous area, while minimizing the effect on the surrounding organs.

Their exploration of branching variable direction selection consists of the three most commonly used options: branch up, branch down, and closest integer. They try these methods with different node selection, variable selection and scaling techniques, as well as with variations on the MIP model itself. The node selection techniques used are depth first, best bound, and best estimate; the variable selection techniques used are least infeasible variable, pseudocosts, and strong branching. Scaling the problem matrix has several different variations: no scaling, standard scaling, and aggressive scaling. Their 
performance measures are CPU time, number of nodes visited and the best integer solution found [Meyer et al. 2003].

Meyer et al. [2003] concludes that the branching direction has a significant impact on solution times. However, there is no overall best branching variable direction method, as each method performed better depending on the scaling method used. For example, they find that branching down works well with aggressive scaling, and branching up works best with standard scaling.

A different study looks at a B\&B algorithm for the dynamic layout problem [Jariwala 1995]. The dynamic layout problem is a problem that assigns the locations of departments in order to minimize the cost of material flow. In this regard, it is a specific application that can be solved by formulating the problem as a MIP and solving it using B\&B. Minimizing cost is the objective function, and the constraints ensure that the departments do not overlap on their layout, and are the correct size. However, the dynamic nature of this problem means it is also desired to minimize the cost of changing from one layout to another.

The branching variable direction selection algorithms compared in this study are: branch up, branch down, and closest integer. Their performance measures are number of nodes visited and final objective function value. Jariwala concludes that for dynamic layout problems, fixing the branching variable direction selection to either branch up or branch down is more effective than using the closest integer heuristic [Jariwala 1995].

One final study tests branching directions with respect to a MIP designed for optimal scrap combination for steel production [Bernatzki et al. 1998]. In this MIP, they want to minimize the cost of using scrap metal to cool the heat produced in steel 
production, while obeying the transportation and material constraints. They test branching up and branching down on their binary variables. By looking at computation time, LP relaxation value, and MIP optimal value, they determined that branching down performs the best for their MIPs. 


\section{Problem Statement}

The goal of this thesis is to find the best branching direction selection heuristic, where the best heuristic is the method that reaches integer feasibility quickly. Methods to be compared include existing methods as well as new methods developed in this thesis.

Running a MIP to feasibility rather than optimality is useful for two reasons: (1) finding a feasible solution enables pruning of the subsequent MIP tree and (2) in some applications of MIPs only feasibility is required.

As discussed in Section 3, there is very little work currently being done to select the best branching variable direction in MIPs. It is generally believed that branching up is a good heuristic to use, although the results in the literature are mixed and yield no firm conclusions. The three application specific studies discussed in Section 3.2 conduct experiments on which branching variable direction selection heuristic is best for their specific niche. However, an overall general analysis of branching variable direction selection heuristics has not been conducted on the set of MIPs. For that reason, one of the intents of this research is to establish the baseline analysis for a general set of MIPs.

Very few branching variable selection algorithms also select the branching variable direction. In fact, only two methods specify which branching direction they select. The active-constraint variable selection methods use branch up arbitrarily and have met success in reaching feasibility quickly [Patel and Chinneck 2007]. The Driebeek and Tomlin method $[1966,1971]$ selects the branching direction based upon their internal degradation calculations. This method is used as the default variable selection method in some MIP solvers [Makhorin 2008], although its success in terms of reaching feasibility quickly is unknown. Improving upon current branching variable 
selection algorithms would also be useful due to their importance in achieving feasibility quickly. In addition, algorithms that simultaneously select the variable and direction are rare. A second goal of this thesis is to develop algorithms of this type and to evaluate their effectiveness. 


\section{New Branching Variable Direction Selection Heuristics}

\subsection{Extension of Counting Solutions Methodology for Direction Selection}

As described in Section 2.2, counting solutions can generate a normal distribution curve given a constraint and the allowable ranges for the variables in the constraint. Knowing the right-hand-side values of the constraint, the curve can provide an estimate of the likelihood the constraint will be satisfied.

In the $\mathrm{B} \& \mathrm{~B}$ algorithm, when a candidate variable is selected, the branching direction must also be selected. By rounding the primal value of the candidate variable up or down to generate the two new bounds, the Counting Solutions methodology can now create two separate normal distributions for each constraint that includes the candidate variable. Based on these two normal distributions, one could show that branching in that direction is more likely to satisfy the constraint than branching in the other direction. In this manner, the normal distribution curves provide a heuristic with which the branching direction decision can be made.

An example should illustrate the above ideas. Using the same constraint as in Section 2.2, which restricts all variables to integer values between 0 and 5 , the original normal distribution curve is shown in Figure 4. After solving the LP relaxation, all three variables have non-integer values. The branching variable selection heuristic selects $x_{l}$, with primal value 1.5 , as the candidate variable. The two bounds added to the two child nodes will restrict $x_{I}$ to $[0,1]$ in the down direction and $[2,5]$ in the up direction. Now a normal distribution curve can be produced for each of the child nodes. The curve for the down direction is shown in Figure 7, and the curve for the up direction is Figure 8. 


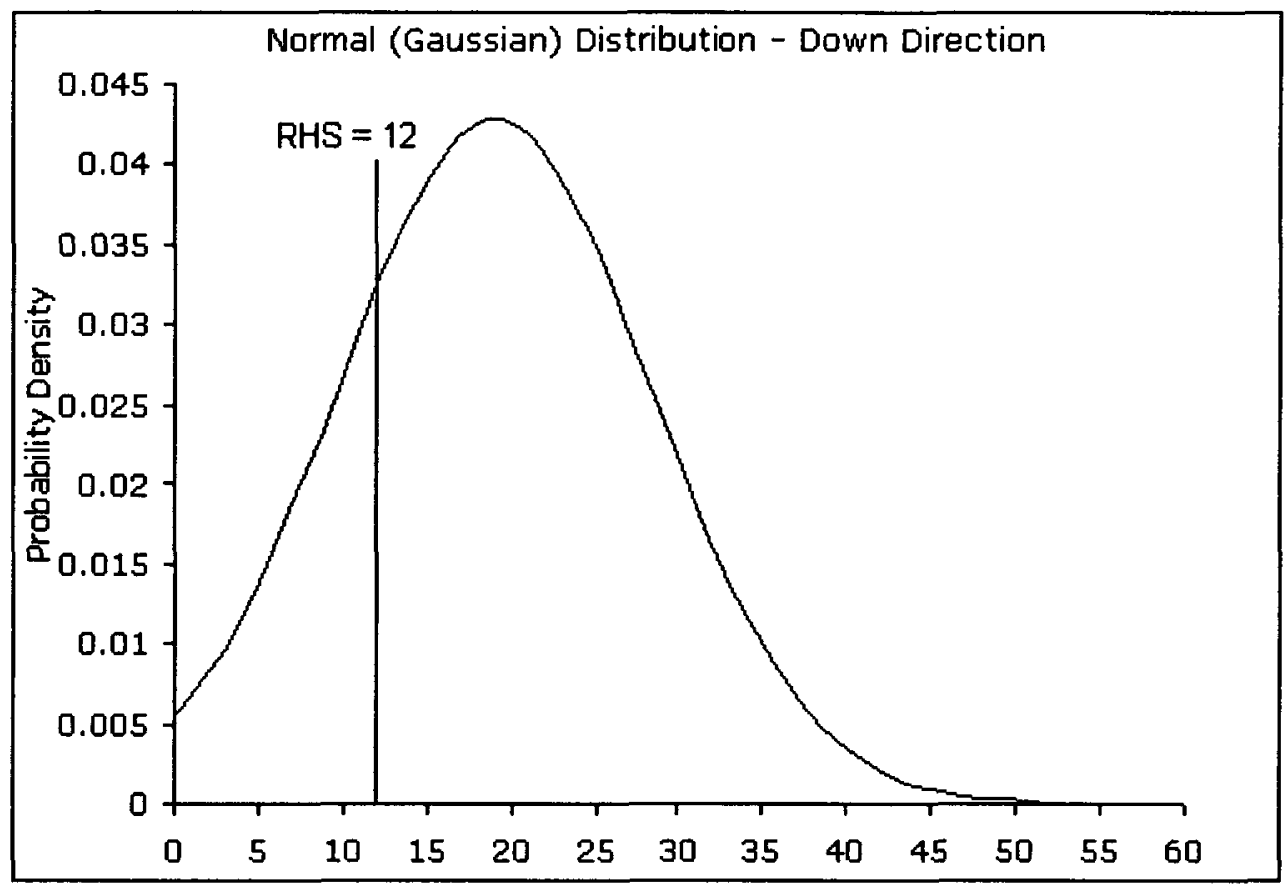

Figure 7. Gaussian curve when branching on $x_{l}$, down direction

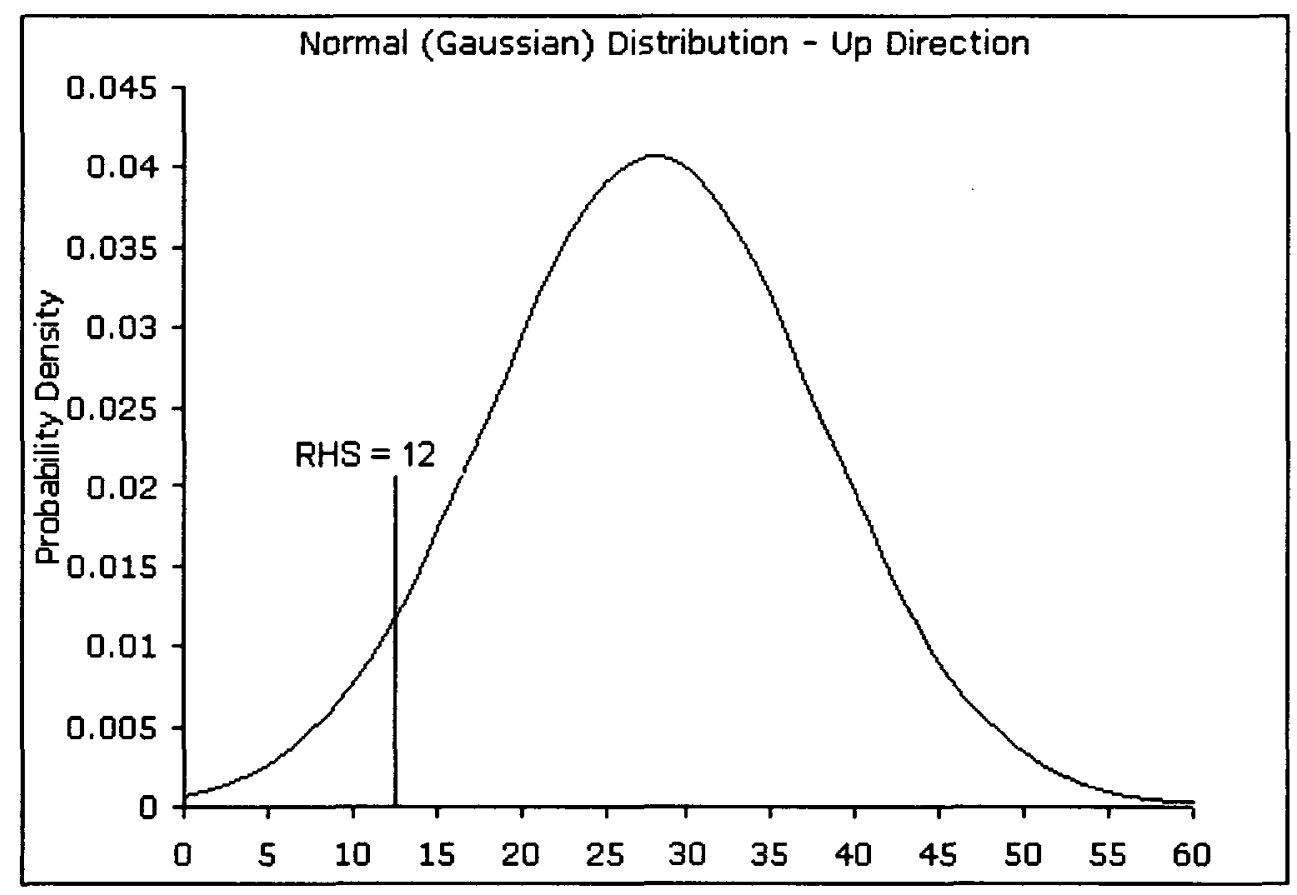

Figure 8. Gaussian curve when branching on $x_{l}$, up direction 
Using the calculations shown in Section 2.2, the down direction cumulative probability is 0.2262 and the up direction cumulative probability is 0.05108 . From this example, it is clear that branching in the down direction is more likely to satisfy the constraint than branching in the up direction on $x_{1}$. The pseudocode in Figure 9 below describes the function GetProbabilities which returns the cumulative probability in the up direction (ProbUp) and the cumulative probability in the down direction (ProbDown). This function is used in several of the new branching direction and variable selection algorithms.

Input: candidate variable called candidate; a constraint the candidate is present in 1. primal $\leftarrow$ LP relaxation optimum value of candidate

2. MeanUp $\leftarrow 0 ;$ MeanDown $\leftarrow 0 ;$ VarUp $\leftarrow 0$; VarDown $\leftarrow 0$ ProbUp $\leftarrow 0$;

ProbDown $\leftarrow 0$

3. rhs $\leftarrow$ RHS value of constraint

4. For each variable in the constraint:

$4.1 \mathrm{lbu} \leftarrow$ current lower bound for the variable in the node $4.2 \mathrm{lbd} \leftarrow$ current lower bound for the variable in the node $4.3 u b u \leftarrow$ current upper bound for the variable in the node $4.4 u b d \leftarrow$ current upper bound for the variable in the node 4.5 coeff $\leftarrow$ coefficient for the variable in this constraint 4.6 If variable $=$ candidate then:

4.6.1 $\mathrm{lbu} \leftarrow$ primal rounded up to the nearest integer

4.6.2 $u b d \leftarrow$ primal rounded down to the nearest integer

4.7 MeanDown $\leftarrow$ MeanDown $+((l b d+u b d) / 2 \times$ coeff $)$

4.8 MeanUp $\leftarrow$ MeanUp $+((l b u+u b u) / 2 \times$ coeff $)$

4.9 VarDown $\leftarrow$ VarDown $+\left(\right.$ coeff $\left.f^{2} \times\left((u b d-l b d+1)^{2}-1\right)\right) / 12$

$4.10 \operatorname{Var} U p \leftarrow \operatorname{Var} U p+\left(\mathrm{coeff} f^{2} \times\left((u b u-l b u+1)^{2}-1\right)\right) / 12$

5. Create a standard normal probability distribution:

5.1 ProbUp $\leftarrow($ rhs - MeanUp $) / \sqrt{\operatorname{VarUp}}$

5.2 ProbDown $\leftarrow($ rhs - MeanDown $) / \sqrt{\text { VarDown }}$

6. ProbUp $\leftarrow$ cumulative probability of ProbUp in standard normal probability distribution (see Section 2.2 for details)

7. ProbDown $\leftarrow$ cumulative probability of ProbDown in standard normal probability distribution (see Section 2.2 for details)

8. Return ProbUp and ProbDown

Figure 9. Pseudocode for GetProbabilities function 
It is possible for the candidate variable $x_{I}$ to be present in several constraints in the MIP. In order to take into account all of the constraints that involve the candidate variable there are two main options: select the absolute "best" probability given by any one constraint the candidate is present in and go in that direction, or assign each constraint a vote based on which direction it thinks is "best", and go in the direction with the most votes.

Next, it is necessary to define what the "best" probability is. Selecting the direction with the highest probability would seem to provide promise of a path that would lead to a feasible, rather than infeasible solution. On the other hand, selecting the direction with the lowest probability could force the B\&B tree to satisfy the hardest constraints and/or variables first. Doing this early in the tree could lead to more future feasible nodes as all the hard decisions have already been made. Branching in the lowest and the highest probability directions both have theoretical merit; therefore both algorithms are included as direction selection algorithms in testing.

\subsubsection{Branching Direction Methods Using Cumulative Probabilities}

In combination with the voting versus absolute "best" probability options, there are four new branching direction selection algorithms to test:

1. Branch in the direction of the absolute lowest cumulative probability returned by one constraint containing the candidate variable. This is referred to as LCP. 
2. Branch in the direction of the absolute highest cumulative probability returned by one constraint containing the candidate variable. This is referred to as HCP.

3. For each constraint containing the candidate variable, decide whether branching up or down gives a lower cumulative probability. Assign a vote in this direction. Branch in the direction with the most votes. This is referred to as LCPV.

4. For each constraint containing the candidate variable, decide whether branching up or down gives a higher cumulative probability. Assign a vote in this direction. Branch in the direction with the most votes. This is referred to as HCPV.

The examples provided to date have dealt with less-than inequalities. For greaterthan inequalities, all that is required is to subtract the calculated cumulative probability from 1. However, equality constraints provide a more difficult challenge. Using a point probability is the most accurate way to represent the probability of exactly satisfying the constraint; however it would not be on the same scale of probabilities as the inequality constraints, making comparisons of lowest and highest probability inaccurate. In a cumulative probability curve, there are an infinite number of points in real space and the probability of landing exactly on a line (as in an equality constraint) is always essentially zero. To assign a value for an equality constraint/candidate variable combination we focus on how "centered" the right-hand-side of the equality constraint is on the probability curve. 
Measuring its centeredness is achieved by taking the ratio of the cumulative probability to the left of the right-hand-side value (of the constraint), versus the cumulative probability to the right of that value. In this manner, if the right-hand-side of the equality constraint had the same value as the mean of the probability curve, the ratio yields a value of 1 . In order to avoid values greater than one, the equality constraint centeredness value is calculated as follows:

$$
\text { EqualityConstraintValue }=\frac{\text { SmallerCumulativeProbability }}{\text { LargerCumulativeProbability }},
$$

where the cumulative probability to the left of the right-hand-side value is compared to the cumulative probability to the right of that value. The smaller of these two probabilities is labelled SmallerCumulativeProbability, and the larger is labelled LargerCumulativeProbability.

The pseudocode for deciding which branching direction is "best" is as shown in Figure 10. Due to the fact that there are four alternatives to the "best" direction, the lowest cumulative probability (LCP) pseudocode is shown below in Figure 10, and the remaining three pseudocodes are in Appendix A.1. 
Input: MIP; candidate variable selected for branching called candidate.

0 . Initialize: LowestProb $\leftarrow 1.1$; Direction $\leftarrow \phi$

1. For each constraint the candidate variable is present in:

1.1 GetProbabilities(candidate, constraint)

1.2 If it is $\mathrm{a} \geq$ constraint:

1.2.1 ProbUp $\leftarrow 1-$ ProbUp; ProbDown $\leftarrow 1-$ ProbDown

1.3 If it is an = constraint:

1.3.1 If ProbUp $>1$ - ProbUp:

1.3.1.1 ProbUp $\leftarrow(1-$ ProbUp $) /$ ProbUp

1.3.2 Else: ProbUp $\leftarrow$ ProbUp / $(1-$ ProbUp $)$

1.3.3 If ProbDown > 1 - ProbDown:

1.3.3.1 ProbDown $\leftarrow(1-$ ProbDown $) /$ ProbDown

1.3.4 Else: ProbDown $\leftarrow$ ProbDown / $(1-$ ProbDown $)$

1.4 Check probabilities (up and down) against current lowest

1.4.1 If ProbUp < LowestProb:

1.4.1.1 LowestProb $\leftarrow$ ProbUp

1.4.1.2 Direction $\leftarrow$ up

1.4.2 If ProbDown < LowestProb:

1.4.2.1 LowestProb $\leftarrow$ ProbDown

1.4.2.2 Direction $\leftarrow$ down

2. Branch in direction Direction.

Figure 10. Pseudocode for lowest cumulative probability direction selection method

\subsection{Satisfied and Violated Voting Methods}

Another heuristic that selects the branching direction is based on the number of constraints that will be satisfied or violated by following a particular branching direction.

We may wish to branch in the direction that keeps the most constraints satisfied for example (or in the direction that violates the most constraints). When looking at just the active inequality constraints, given the branching variable, a direction vote can be generated by each constraint. This is achieved by looking at the sign of the coefficient of the branching variable in that constraint, as well as the sense of the constraint (less-than, or greater-than). 
As an example, say the coefficient is positive, the constraint is less-than, and it is assumed that all other variables in the constraint remain fixed at their current values. If the up direction is selected, the branching variable value will increase and the constraint will be violated. On the other hand, by selecting the down direction, the branching variable value decreases and the constraint remains satisfied. The eight possibilities for satisfied and violated constraints are outlined in Table 2 below.

Table 2. Combinations possible for violated and satisfied constraints

\begin{tabular}{|c|c|c|c|}
\hline Coefficient Sign & Constraint Sense & Branching Direction & Result \\
\hline+ & $\leq$ & up & violated \\
\hline+ & $\leq$ & down & satisfied \\
\hline+ & $\geq$ & up & satisfied \\
\hline+ & $\leq$ & down & violated \\
\hline- & $\leq$ & up & satisfied \\
\hline- & $\leq$ & down & violated \\
\hline- & $\geq$ & up & violated \\
\hline- & $\geq$ & down & satisfied \\
\hline
\end{tabular}

This method only works for inequalities. In order to deal with equality constraints the following measures are implemented. An equality constraint will always be violated when a change is made to a variable present in that constraint (assuming that all other variables retain their values), so a heuristic counting satisfied versus violated direction votes will not work. For this reason, we return to the idea of cumulative probabilities. Rather than using the same equalities ratio discussed in Section 5.1.1, two different methods for voting based on equalities are used to keep in the spirit of the violated/satisfied approach. There are four cumulative probabilities in total. There are two probability curves, one for the up direction, one for the down direction, and a cumulative probability can be calculated in each curve based upon where the right-hand- 
side of the constraint falls. Subtracting that cumulative probability from 1 provides the second probability for the curve. These probabilities can be classified as:

1. down direction probability curve, probability of being below the RHS value (DB)

2. down direction probability curve, probability of being above the RHS value (DA)

3. up direction probability curve, probability of being below the RHS value (UB)

4. up direction probability curve, probability of being above the RHS value (UA)

Among these four, the probability that represents the greatest violation of the constraint is the smallest of the probabilities. This is also the direction given by the least centred RHS value, which is why it leads to the largest chance that the constraint will be violated. Similarly for the satisfied vote, we select the direction that has the RHS that is most centered. This can be decided by looking at the minimum cumulative probability in the up branching direction curve, and comparing it to the minimum cumulative probability in the down branching direction curve. The larger of these two minima represents the most centered RHS, and therefore the best chance of satisfying the constraint.

To summarize, if the constraint is an equality constraint, the satisfied/violated direction vote is assigned as follows: calculate the four cumulative probabilities and compare them. For violated votes, add a vote to the branching direction with the smallest cumulative probability, or

$$
\min (\mathrm{DB}, \mathrm{DA}, \mathrm{UB}, \mathrm{UA}) \text {. }
$$


For satisfied votes, add a vote to the branching direction with the largest of the two smallest cumulative probabilities (in their respective branching directions), or

$$
\max [\min (D B, D A), \min (U B, U A)] .
$$

The active constraints are the only constraints allowed to vote, because they are the ones that have the most effect on the change to the LP solution. Also, the active constraints are the ones whose violated/satisfied status can be affected by altering the value of a single variable (if it is an inequality). The votes are then tallied, and the winning direction is selected as the branching direction. If the votes are tied, then the algorithm defaults to the up branching direction.

\subsubsection{Branching Direction Methods Using Violated and Satisfied Voting Methods}

The pseudocode for the violated votes algorithm (MVV) is shown below in Figure 11. The satisfied votes algorithm (MSV) is in Appendix A.2 for completeness. 
Input: MIP; candidate variable selected for branching called candidate.

0 . Initialize: Tally $U p \leftarrow 0$; TallyDown $\leftarrow 0$;

1. For each constraint the candidate is present in:

1.1 If constraint is active:

1.1.1 coeff $\leftarrow$ coefficient for candidate in constraint

1.1.2 If constraint is an equality:

1.1.2.1 GetProbabilities(candidate, constraint)

1.1.2.2 If ProbDown >0.5:

1.1.2.2.1 ProbDown $\leftarrow(1-$ ProbDown $)$

1.1.2.3 If ProbUp >0.5:

1.1.2.3.1 ProbUp $\leftarrow(1-$ ProbUp $)$

1.1.2.4 If ProbDown < ProbUp:

1.1.2.4.1 Increment TallyDown

1.1.2.5. Else: Increment TallyUp

1.1.3 Else if constraint is a less-than inequality:

1.1.3.1 If coeff is positive:

1.1.3.1.1 Increment TallyUp

1.1.3.2 Else: Increment TallyDown

1.1.4 Else:

1.1.4.1 If coeff is positive:

1.1.4.1.1 Increment TallyDown

1.1.4.2 Else: Increment Tally $U p$

2. If TallyDown is larger than TallyUp: branch down

3. Else: branch up.

Figure 11. Pseudocode for violated votes branching direction selection method

\subsection{Extension of Counting Solutions Methodology for Simultaneous Variable and Direction Selection}

The method described in Section 5.1 can be further extended to select the branching variable as well as the branching direction. This can be achieved by adding a few steps prior to Step 1 in Figure 10.

First of all, the candidate variable is not yet selected, so that is not an input into the algorithm. Instead, cycle through the list of constraints in the MIP, looking at each variable in the constraint. If the current variable is a candidate variable, the "best" probability for that constraint-variable combination is calculated and compared to the current "best" (Steps 1.1 - 1.4.2.2 in Figure 10). In addition to storing the direction that is 
"best", store which candidate variable produced the "best" probability. In this manner, after all the constraints have been examined, the value stored in the "best" probability indicates which candidate variable, and direction should be selected for branching.

\subsubsection{Simultaneous Variable and Direction Selection Methods Using Cumulative Probabilities}

The full version of the lowest cumulative probability variable and direction selection algorithm (VDS-LCP) can be found in Figure 12 below. This algorithm is implemented and compared against the state of the art in branching variable selection algorithms in Experiment 2, which is in Section 8 of this document. 
Input: MIP

0 . Initialize: LowestProb $\leftarrow 1.1 ;$ Direction $\leftarrow \phi ;$ branch-candidate $\leftarrow \phi$

1. For each constraint in the MIP:

1.1 For each variable in the constraint:

1.1.1 If the variable is candidate variable candidate then:

1.1.1.1 GetProbabilities (candidate, constraint)

1.1.1.2 If it is a $\geq$ constraint:

1.1.1.2.1 ProbUp $\leftarrow 1-$ ProbUp; ProbDown $\leftarrow 1-$ ProbDown

1.1.1.3 If it is an = constraint:

1.1.1.3.1 If ProbUp > 1 - ProbUp:

1.1.1.3.1.1 ProbUp $\leftarrow(1-$ ProbUp $) /$ ProbUp

1.1.1.3.2 Else: ProbUp $\leftarrow$ ProbUp / (1 - ProbUp $)$

1.1.1.3.3 If ProbDown > 1 - ProbDown:

1.1.1.10.3.1 ProbDown $\leftarrow(1-$ ProbDown $) /$

ProbDown

1.1.1.3.4 Else: ProbDown $\leftarrow$ ProbDown $/(1-$

ProbDown)

1.1.1.4 Check probabilities (up and down) against current lowest

1.1.1.4.1 If ProbUp < LowestProb:

1.1.1.4.1.1 LowestProb $\leftarrow$ ProbUp

1.1.1.4.1.2 branch-candidate $\leftarrow$ candidate

1.1.1.4.1.3 Direction $\leftarrow$ up

1.1.1.4.2 If ProbDown < LowestProb:

1.1.1.4.2.1 LowestProb $\leftarrow$ ProbDown

1.1.1.4.2.2 branch-candidate $\leftarrow$ candidate

1.1.1.4.2.3 Direction $\leftarrow$ down

2. Perform branching on branch-candidate in direction direction.

Figure 12. Pseudocode for variable and direction selection using lowest cumulative probability method

Again, the "best" probability can mean a variety of things. In addition to algorithms selecting the absolute lowest and highest probabilities, other variations are available. This includes selecting the candidate variable with the lowest or highest probability, but then branching in the opposite direction. A summary of the four 
cumulative probability variable and direction selection methods tested in Experiment 2 is shown below in Table 3 .

Table 3. Simultaneous variable and direction selection methods using cumulative probabilities

\begin{tabular}{|c|l|}
\hline $\begin{array}{c}\text { Method } \\
\text { Name }\end{array}$ & \multicolumn{1}{c|}{ Brief Explanation } \\
\hline VDS-LCP & Select variable and direction of lowest cumulative probability \\
\hline VDS-HCP & Select variable and direction of highest cumulative probability \\
\hline VDS-OLCP & $\begin{array}{l}\text { Select variable of lowest cumulative probability, branch in opposite } \\
\text { direction }\end{array}$ \\
\hline VDS-OHCP & $\begin{array}{l}\text { Select variable of highest cumulative probability, branch in opposite } \\
\text { direction }\end{array}$ \\
\hline
\end{tabular}

\subsubsection{Simultaneous Variable and Direction Selection Methods Using Point Probabilities}

In addition, the point probability of a particular RHS value can be calculated. This value represents the probability of directly hitting the RHS value of a constraint, given the branching variable. In point probabilities, all calculations are the same regardless of the type of constraint (greater-than, less-than inequality, or equality). The pseudocode for this algorithm is shown in Figure 13 below. Point probabilities are calculated using the following formula:

$$
\frac{1}{\sigma \sqrt{2 \pi}} \exp \left(-\frac{(R H S-\mu)^{2}}{2 \sigma^{2}}\right)
$$

where $\mu$ is the mean, $\sigma$ is the variance, and $R H S$ is the right-hand-side value of the current constraint. 
Input: MIP

0 . Initialize: LowestProb $\leftarrow 1.1$; Direction $\leftarrow \phi ;$ branch-candidate $\leftarrow \phi$

1. For each constraint in the MIP:

1.1 For each variable in the constraint:

1.1.1 If the variable is candidate variable candidate then:

1.1.1.1 primal $\leftarrow$ LP relaxation optimum value of candidate

1.1.1.2 MeanUp $\leftarrow 0$; MeanDown $\leftarrow 0$; VarUp $\leftarrow 0$;

VarDown $\leftarrow 0 ;$ ProbUp $\leftarrow 0 ;$ ProbDown $\leftarrow 0$

1.1.1.3 rhs $\leftarrow$ RHS value of constraint

1.1.1.4 For each variable in the constraint:

1.1.1.4.1 $l b u \leftarrow$ current lower bound for the variable in the node

1.1.1.4.2 lbd $\leftarrow$ current lower bound for the variable in the node

1.1.1.4.3 $u b u \leftarrow$ current upper bound for the variable in the node

1.1.1.4.4 ubd $\leftarrow$ current upper bound for the variable in the node

1.1.1.4.5 coeff $\leftarrow$ coefficient for the variable in this constraint

1.1.1.4.6 If variable $=$ candidate then:

1.1.1.4.6.1 lbu $\leftarrow$ primal rounded up to the nearest integer

1.1.1.4.6.2 ubd $\leftarrow$ primal rounded down to the nearest integer

1.1.1.4.7 MeanDown $\leftarrow$ MeanDown $+(($ lbd $+u b d) / 2 \times$ coeff $)$

1.1.1.4.8 MeanUp $\leftarrow$ MeanUp $+((l b u+u b u) / 2 \times$ coeff $)$

1.1.1.4.9 VarDown $\leftarrow$ VarDown +

$\left(\right.$ coeff $\left.{ }^{2} \times\left((u b d-l b d+1)^{2}-1\right)\right) / 12$

1.1.1.4.10 VarUp $\leftarrow \operatorname{Var} U p+\left(\right.$ coeff $\left.{ }^{2} \times\left((u b u-l b u+1)^{2}-1\right)\right) / 12$

1.1.1.5 Compute ProbUp and ProbDown as in Equation (3).

1.1.1.6 Check probabilities (up and down) against current lowest

1.1.1.6.1 If ProbUp < LowestProb:

1.1.1.6.1.1 LowestProb $\leftarrow$ ProbUp

1.1.1.6.1.2 branch-candidate $\leftarrow$ candidate

1.1.1.6.1.3 Direction $\leftarrow$ up

1.1.1.6.2 If ProbDown < LowestProb:

1.1.1.6.2.1 LowestProb $\leftarrow$ ProbDown

1.1.1.6.2.2 branch-candidate $\leftarrow$ candidate

1.1.1.6.2.3 Direction $\leftarrow$ down

2. Perform branching on branch-candidate in direction direction.

Figure 13. Pseudocode for variable and direction selection using lowest point probability method 
The point probabilities can be calculated and used in the usual variations of lowest/highest/opposite of lowest/opposite of highest probabilities. Table 4 below summarizes the four point probability variable and direction selection methods tested in Experiment 2.

Table 4. Simultaneous variable and direction selection methods using point probabilities

\begin{tabular}{|c|l|}
\hline Method Name & \multicolumn{1}{c|}{ Brief Explanation } \\
\hline VDS-LPP & Select variable and direction of lowest point probability \\
\hline VDS-HPP & Select variable and direction of highest point probability \\
\hline VDS-OLPP & Select variable of lowest point probability, branch in opposite direction \\
\hline VDS-OHPP & Select variable of highest point probability, branch in opposite direction \\
\hline
\end{tabular}

\subsubsection{Simultaneous Variable and Direction Selection Methods Using Multiplied Probabilities}

A final method is generating one probability per candidate variable by multiplying together all of the probabilities from each constraint the variable is present in. The multiplied value is then used for comparison purposes. A summary table of the eight multiplied probability variable and direction selection methods tested in Experiment 2 is provided below in Table 5. There are eight methods here because we use both cumulative and point probabilities. 
Table 5. Simultaneous variable and direction selection methods using multiplied probabilities

\begin{tabular}{|c|l|}
\hline Method Name & \multicolumn{1}{c|}{ Brief Explanation } \\
\hline VDS-LCPM & $\begin{array}{l}\text { Select variable and direction of lowest multiplied cumulative } \\
\text { probability }\end{array}$ \\
\hline VDS-HCPM & $\begin{array}{l}\text { Select variable and direction of highest multiplied cumulative } \\
\text { probability }\end{array}$ \\
\hline VDS-OLCPM & $\begin{array}{l}\text { Select variable of lowest multiplied cumulative probability, branch in } \\
\text { opposite direction }\end{array}$ \\
\hline VDS-OHCPM & $\begin{array}{l}\text { Select variable of highest multiplied cumulative probability, branch } \\
\text { in opposite direction }\end{array}$ \\
\hline VDS-LPPM & Select variable and direction of lowest multiplied point probability \\
\hline VDS-HPPM & Select variable and direction of highest multiplied point probability \\
\hline VDS-OLPPM & $\begin{array}{l}\text { Select variable of lowest multiplied point probability, branch in } \\
\text { opposite direction }\end{array}$ \\
\hline VDS-OHPPM & $\begin{array}{l}\text { Select variable of highest multiplied point probability, branch in } \\
\text { opposite direction }\end{array}$ \\
\hline
\end{tabular}

\subsubsection{Simultaneous Variable and Direction Selection Methods Using Hybrid Methods}

Early testing also indicated that five additional hybrids of probability methods were worth pursuing. These algorithms are listed in Table 6 below. In particular the presence of equality constraints in the MIP affects the performance of the algorithms (HYBRIDI). Also the presence of candidate variables in both inequality and equality constraints affects the performance of the algorithms (HYBRID2). As discussed later in Experiment 1 , branching up is a good branching direction heuristic. This rationale is used for hybrids $V D S-L C P-U P 1$ and VDS-LCP-UP2. In general, early experiments showed METHA performs very well, which is why the hybrid methods are built on it.

One issue that has not been discussed to date is the number of ties amongst the candidate variables when selecting the branching variable. Both VDS-LCP and the METHA have a significant number of ties for branching variable and direction, however 
both of their algorithms select the first "best" candidate it encounters as the branching variable. An exploration of the ties for branching variable amongst VDS-LCP and METHA reveals a significant common subset of ties for VDS-LCP and METHA. This revelation led to a hybrid, called TIES. At each node, this method looks at the lists of tied candidate variables for VDS-LCP and METHA. If the two lists have any common candidate variables, the first of those common candidate variables is selected as the branching variable, using the up branching direction. However, if there are no common ties, METHA's candidate variable is used as the branching variable.

Table 6. Simultaneous variable and direction selection methods using hybrids

\begin{tabular}{|c|l|}
\hline Method Name & \multicolumn{1}{c|}{ Brief Explanation } \\
\hline HYBRID1 & $\begin{array}{l}\text { Use VDS-LCP if there are equality constraints in the MIP, otherwise } \\
\text { use METHA. }\end{array}$ \\
\hline TIES & $\begin{array}{l}\text { If VDS-LCP and METHA have any common ties for branching } \\
\text { variable, choose the first variable in the common ties list and branch } \\
\text { up. Otherwise, use METHA's original selection. }\end{array}$ \\
\hline VDS-LCP-UP1 & $\begin{array}{l}\text { Use VDS-LCP to select the candidate variable and then branch in the } \\
\text { up direction. }\end{array}$ \\
\hline VDS-LCP-UP2 & $\begin{array}{l}\text { If there are equality constraints in the MIP use VDS-LCP, otherwise } \\
\text { use VDS-LCP-UP1. }\end{array}$ \\
\hline HYBRID2 & $\begin{array}{l}\text { Use VDS-LCP if the current node has candidate variables present in } \\
\text { both equality and inequality constraints, otherwise use METHA. }\end{array}$ \\
\hline
\end{tabular}




\section{Experimental Setup}

\subsection{Software}

The GNU Linear Programming Kit (GLPK) Version 4.28 [Makhorin 2008] was modified to test the various branching direction and variable selection algorithms. GLPK was selected as the MIP solver because it is open-source, has excellent documentation, help forums, and user feedback mechanisms. GLPK is programmed in ANSI C, and it was compiled using the Dev-C++ Version 4.9.9.2 compiler [Laplace 2009]. Numerous modifications were made to GLPK 4.28.

\subsection{Parameter Settings}

The stopping conditions were set as follows: MIPs were run until they reached the first feasible node, or until a two hour time limit was exceeded. The limit of two hours is common in MIP research [Patel and Chinneck 2007]. Changing the time limit was performed by the following GLPK command: lpx_set_real_parm(lp, LPX_K_TMLIM, 7200). To exit after a feasible node was found, the GLPK command "goto done" was used if the solution was integer feasible. This line was added after GLPK checked the integrality of the LP relaxation. The remaining modifications to GLPK are as follows below:

- Aside from the tests run on the default GLPK method, the node selection technique was forced to be depth-first for all tests. This was performed by the following GLPK command: lpx_set_int_parm(lp, LPX_K_BTRACK, 0).

- The branching variable selection and branching direction techniques were changed from their defaults to what the current experiment was testing. This was 
performed by the following GLPK command: lpx_set_int_parm(lp,

LPX_K_BRANCH, branchtech).

\subsection{Hardware}

Three computers running Windows XP were used for the experiments:

- A Pentium 4 CPU with $3.40 \mathrm{GHz}$ and $1 \mathrm{~GB}$ of RAM

- An Intel Core $2 \mathrm{CPU}$ with $3.40 \mathrm{GHz}$ and 3GB of RAM

- A Pentium 4 CPU with $3.20 \mathrm{GHz}$ and $3 \mathrm{~GB}$ of RAM

Because the three computers have different specifications, any methods compared were run on the same machine.

\subsection{Test Set}

The following MIP problem sets were used: MIPLIB2003 [Achterberg et al. 2006], MIPLIB3.0 [Bixby et al. 1998], MIPLIB2.0 [Bixby et al. 1992], and Coral [Linderoth 2009]. These problems sets are used frequently in the evaluation of MIP solvers, and new B\&B heuristics because of the variety of real-world problems they cover. They are representative of a good general set of MIPs [Achterberg et al. 2006].

Equality constraints are treated differently in some of the heuristics and for this reason the MIP test models were separated into two groups: those having no equality constraints, and those having one or more equality constraints. The former set is referred to as the "equality-free MIP set" and the latter is referred to as the "equality-containing MIP set" for the remainder of this thesis.

MIPLIB2003 was the primary source of MIP models for these experiments. It contains a total of sixty MIPs, fifty-one of which are classified in the equality-containing 
MIP set. These fifty-one equality-containing MIPs from MIPLIB2003 make up the entire equality-containing MIP set (see list in Appendix A.3). The remaining nine MIPLIB2003 MIPs were added to the equality-free MIP set. To augment this problem set, all of the equality-free MIPs were taken from MIPLIB3.0, MIPLIB2.0, and Coral giving a total of ninety-five equality-free MIPs (see list in Appendix A.4).

\subsection{Algorithms Compared}

In Experiment 2 all of the new variable and direction selection algorithms were tested against the branching variable selection algorithms available in GLPK as well as METHA, which is considered state of the art in quickly finding the first integer-feasible solution for a MIP [Patel and Chinneck 2007]. The default node selection technique in GLPK is best local bound. GLPK's default branching variable selection technique is the Driebeek and Tomlin heuristic. When an algorithm is compared against GLPKDEFAULT, it is implied that it was compared against the Driebeek and Tomlin heuristic with best local bound node selection. All of the other algorithms compared use depth first node selection. The four branching variable selection algorithms built into GLPK are: most fractional (GLPK1), first fractional (GLPK2), last fractional (GLPK3), and the Driebeek and Tomlin Heuristic (GLPK4).

As described in Section 3, each of these methods has an associated branching direction heuristic in GLPK. METHA uses branch up when it is described in the literature, and therefore this convention was adopted when it was implemented in GLPK. Table 7 lists the six state of the art methods that were used for comparisons in Experiment 2. 
Table 7. List of existing methods compared against in Experiment 2

\begin{tabular}{|l|l|l|l|}
\hline Method Name & Variable Selection & Node Selection & $\begin{array}{l}\text { Branching } \\
\text { Direction }\end{array}$ \\
\hline GLPK1 & Most Fractional & Depth First & Closest Integer \\
\hline GLPK2 & First Fractional & Depth First & Closest Integer \\
\hline GLPK3 & Last Fractional & Depth First & Closest Integer \\
\hline GLPK4 & Driebeek \& Tomlin & Depth First & Driebeek \& Tomlin \\
\hline GLPKDEFAULT & Driebeek \& Tomlin & $\begin{array}{l}\text { Best Local } \\
\text { Bound }\end{array}$ & Driebeek \& Tomlin \\
\hline METHA & $\begin{array}{l}\text { Active Constraints Method } \\
\text { A }\end{array}$ & Depth First & Up Always \\
\hline
\end{tabular}

In Experiment 2, the above six methods are compared to the methods in Tables 3-

6. However in Experiment 1 the above six methods, except for the branching direction column, are run using nine different branching direction heuristics. This will be explained in depth in Section 7.

\subsection{Performance Metrics}

In order to accurately compare one algorithm to the next, three performance metrics were collected: time, number of nodes evaluated, and the number of simplex iterations completed. All three metrics were plotted on performance profiles over the MIP problem set. For more information about performance profiles and how they are generated refer to Section 2.3 or [Dolan and Moré 2002]. All three performance metrics are generally considered the standards for MIP algorithmic comparisons. The number of dives required to reach the first feasible solution was also analyzed for the variable selection algorithms as it is a good indicator of the quality of the algorithm [Patel and Chinneck 2007]. The number of dives can also be thought of as the number of infeasible leaf nodes reached before a feasible leaf node is found. 
In the results, there were instances where the computation time metric was 0 or close to 0 . This leads to an infeasible ratio to best for the performance profiles. For this reason any times that were less than 1 second were assigned a ratio to best of 1 , and the other methods being compared against it were modified accordingly. This also avoids inaccurately large ratios to best for other methods being compared to the fast method. 


\section{Experiment 1: Selecting the Branching Direction}

In order to evaluate the effectiveness of new and existing branching variable direction selection heuristics, two experiments were carried out. Sections 7 and 8 describe these experiments in detail along with their results. The goal of the first experiment is to determine if there is an overall best branching variable direction selection heuristic. It also tests whether the folklore about branching up being a superior heuristic is true. The second experiment explores an algorithm that simultaneously selects the branching variable and direction via the modified Counting Solutions probability method proposed in this thesis. This algorithm is compared to other state of the art branching variable selection algorithms to determine its merits. To summarize:

- Experiment 1: Given the branching variable by some method, which direction heuristic is best?

- Experiment 2: Simultaneous variable and direction selection.

\subsection{Goal of Experiment}

The goal of Experiment 1 is to determine what the best branching variable direction selection heuristic is under a variety of node and variable selection situations. Nine branching variable direction selection heuristics are tested. They are listed in Table 8 below. 
Table 8. Branching variable direction selection heuristics compared in Experiment 1

\begin{tabular}{|l|l|}
\hline Method Acronym & Name \\
\hline UP & Branch Up (Section 3.1) \\
\hline DOWN & Branch Down (Section 3.1) \\
\hline CI & Closest Integer (Section 3.1) \\
\hline LCP & Lowest Cumulative Probability (Section 5.1.1) \\
\hline HCP & Highest Cumulative Probability (Section 5.1.1) \\
\hline LCPV & Lowest Cumulative Probability Votes (Section 5.1.1) \\
\hline HCPV & Highest Cumulative Probability Votes (Section 5.1.1) \\
\hline MSV & Most Satisfied Votes (Section 5.2.1) \\
\hline MVV & Most Violated Votes (Section 5.2.1) \\
\hline
\end{tabular}

Each branching variable direction heuristic is run using the six combinations of variable and node selection methods listed in Table 9 below. The heuristics are run on all 95 equality-free MIPs and 47 of the 51 equality-containing MIPs (Momentum3.mps, msc98-ip.mps, rd-rplusc-21.mps, and stp3d.mps are omitted because they timed out in all of the branching variable selection methods used in this experiment).

Table 9. Variable and node selection algorithms used in Experiment 1

\begin{tabular}{|l|l|l|}
\hline Method Name & Variable Selection & Node Selection \\
\hline GLPK1 & Most Fractional & Depth First \\
\hline GLPK2 & First Fractional & Depth First \\
\hline GLPK3 & Last Fractional & Depth First \\
\hline GLPK4 & Driebeek \& Tomlin & Depth First \\
\hline GLPKDEFAULT & Driebeek \& Tomlin & Best Local Bound \\
\hline METHA & Active Constraints Method A & Depth First \\
\hline
\end{tabular}

\subsection{Experimental Results}

Each performance profile below summarizes the results for one of the three metrics (time, number of nodes, or simplex iterations). The data is separated by the six combinations of branching variable and node selection methods to see if any branching direction heuristic is a better fit to a particular variable selection/node selection combination. These separations show that branching up is always the top branching direction method 
regardless of the variable selection/node selection combination. Graphs showing the separation of the data by variable selection/node selection combination are shown in the next eighteen figures.

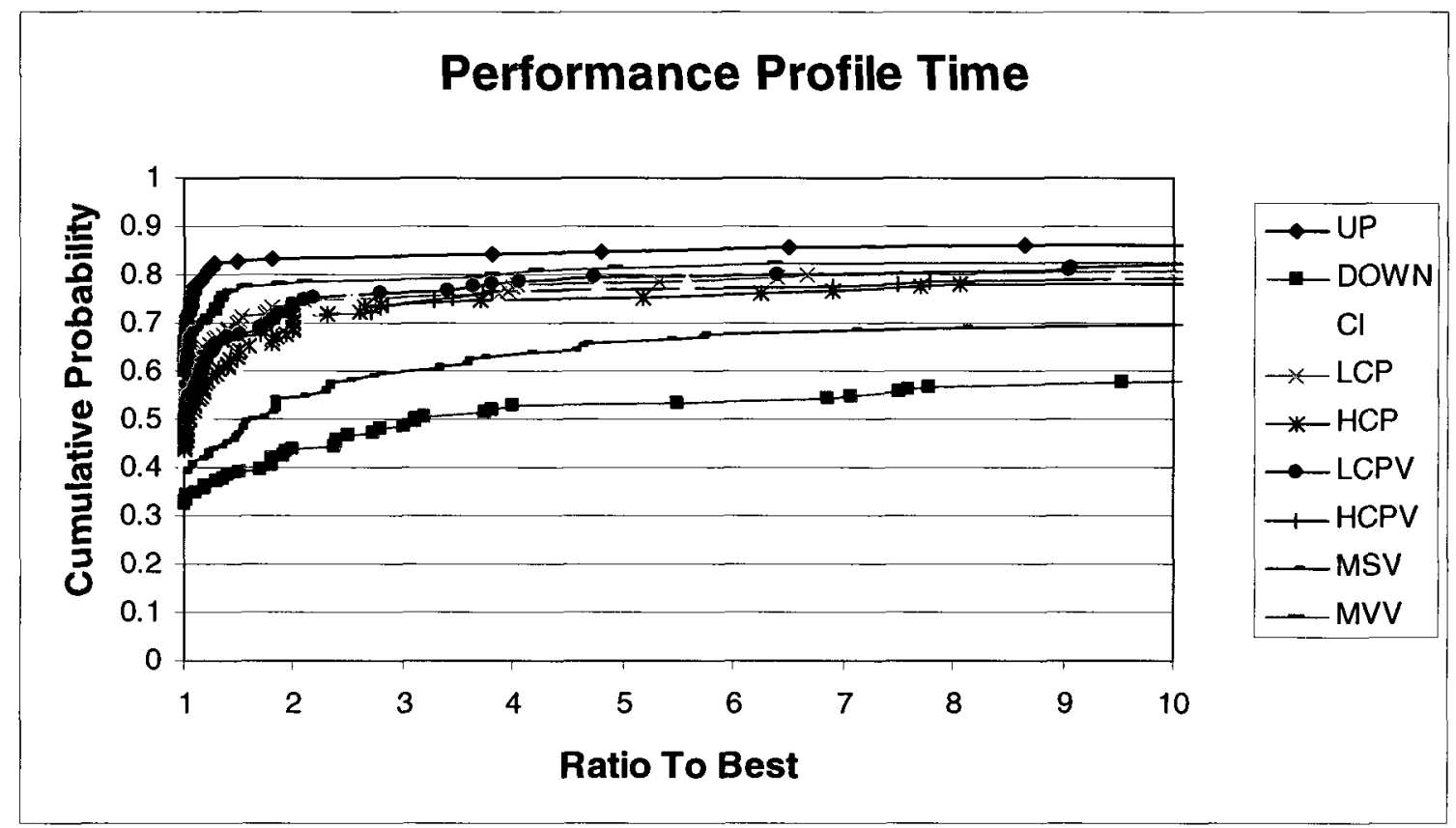

Figure 14. Experiment 1 performance profile for GLPK1 - computation time

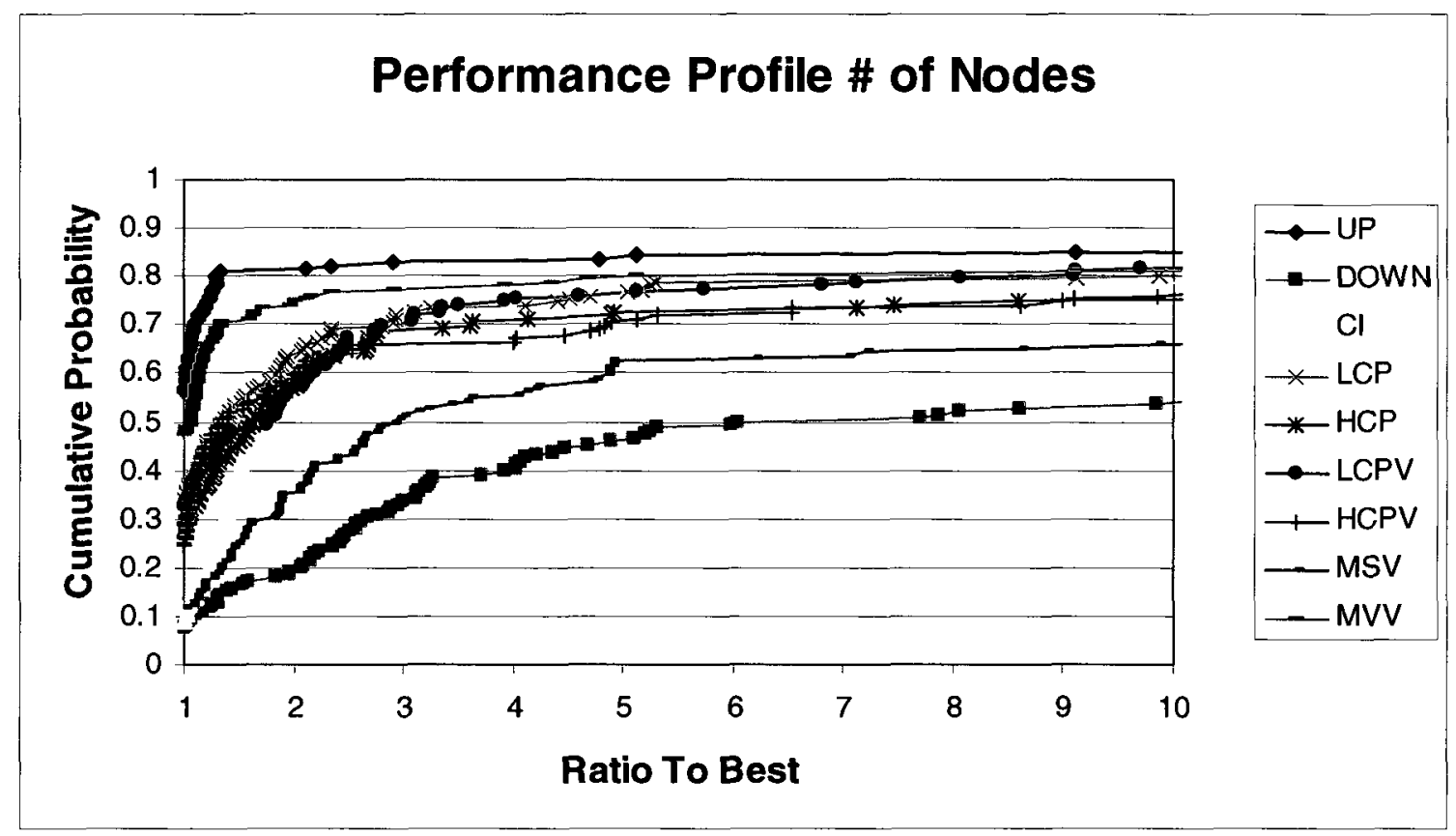

Figure 15. Experiment 1 performance profile for GLPK1 - number of nodes 


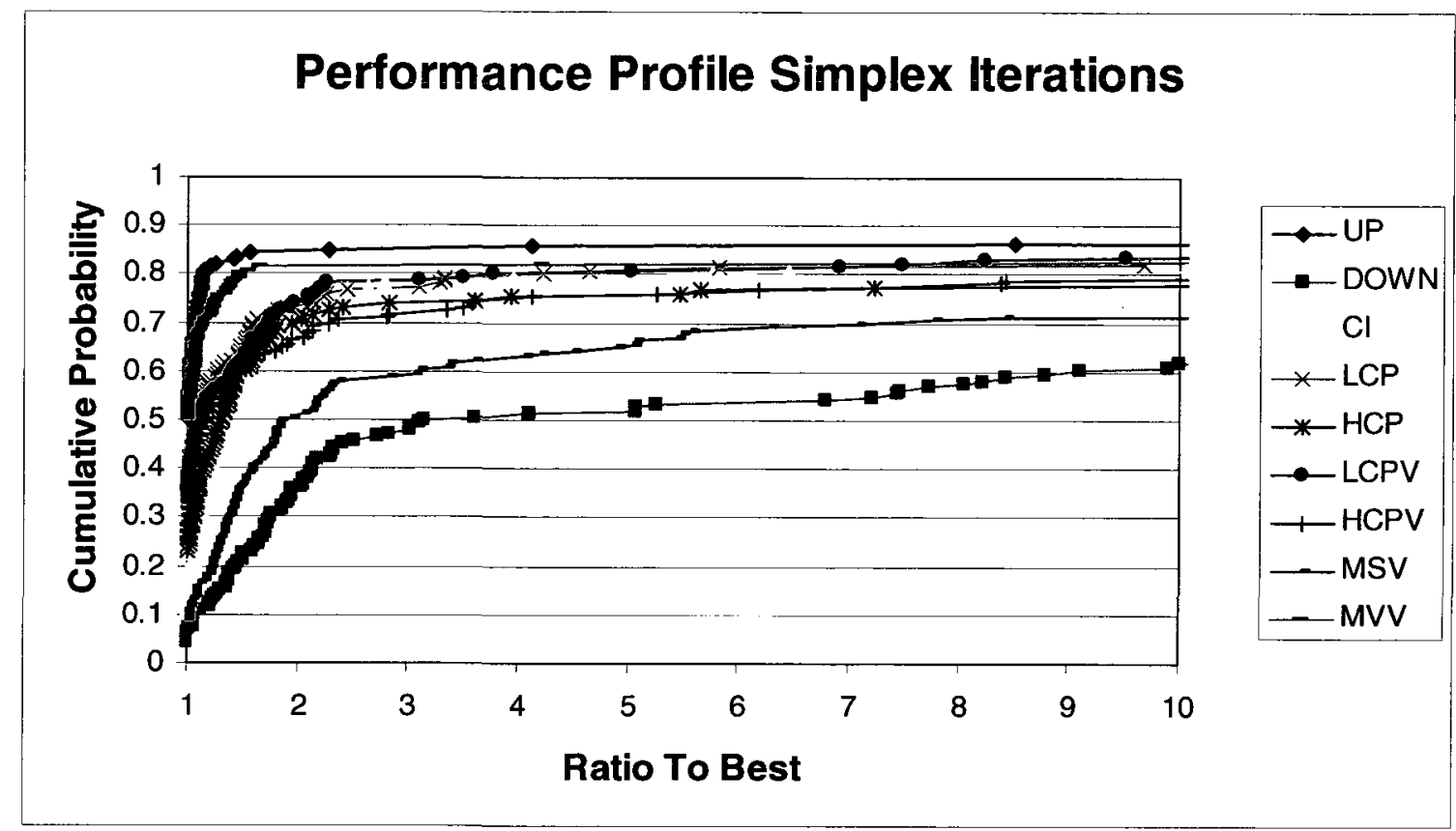

Figure 16. Experiment 1 performance profile for GLPK1 - simplex iterations

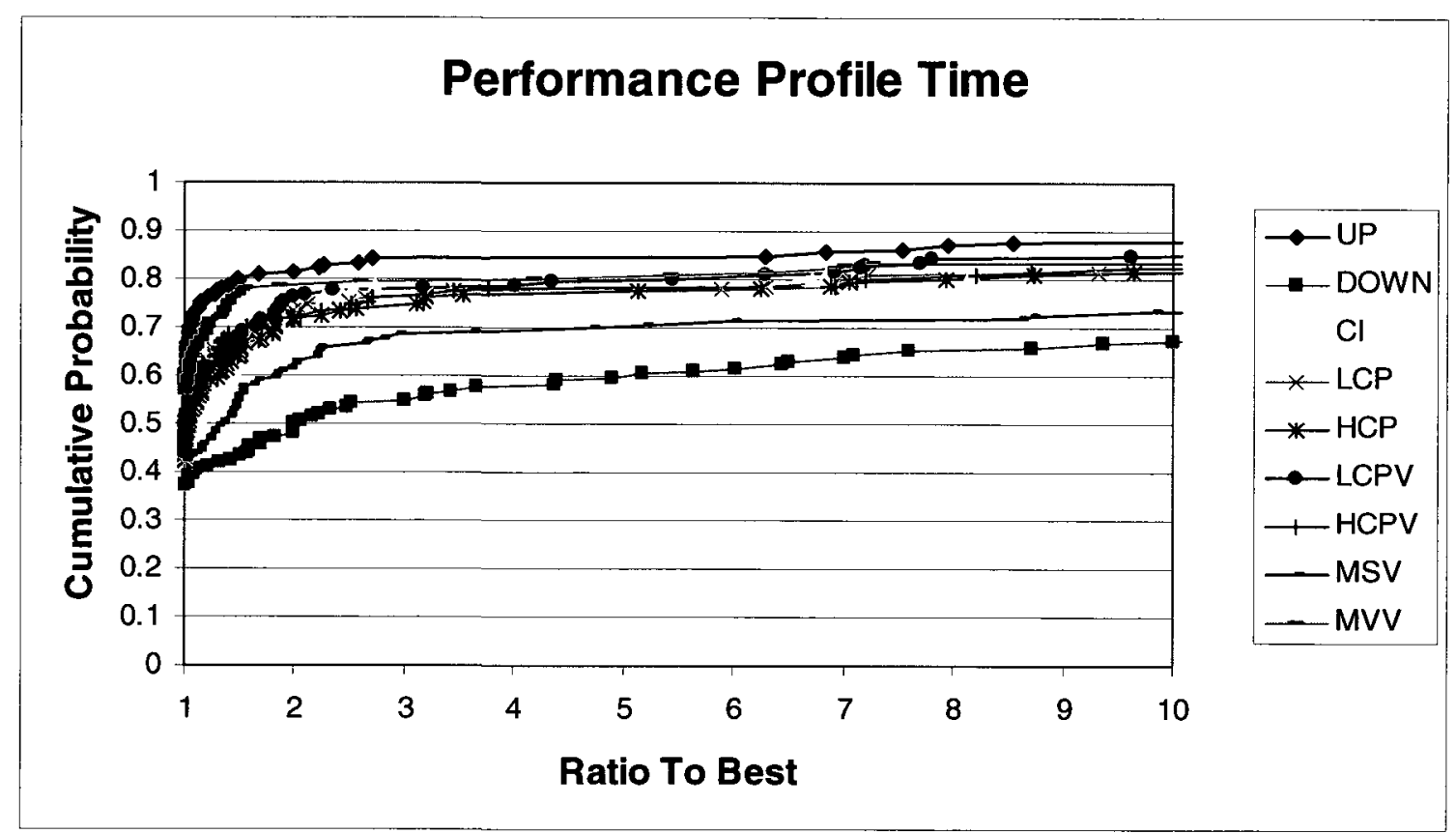

Figure 17. Experiment 1 performance profile for GLPK2 - computation time 


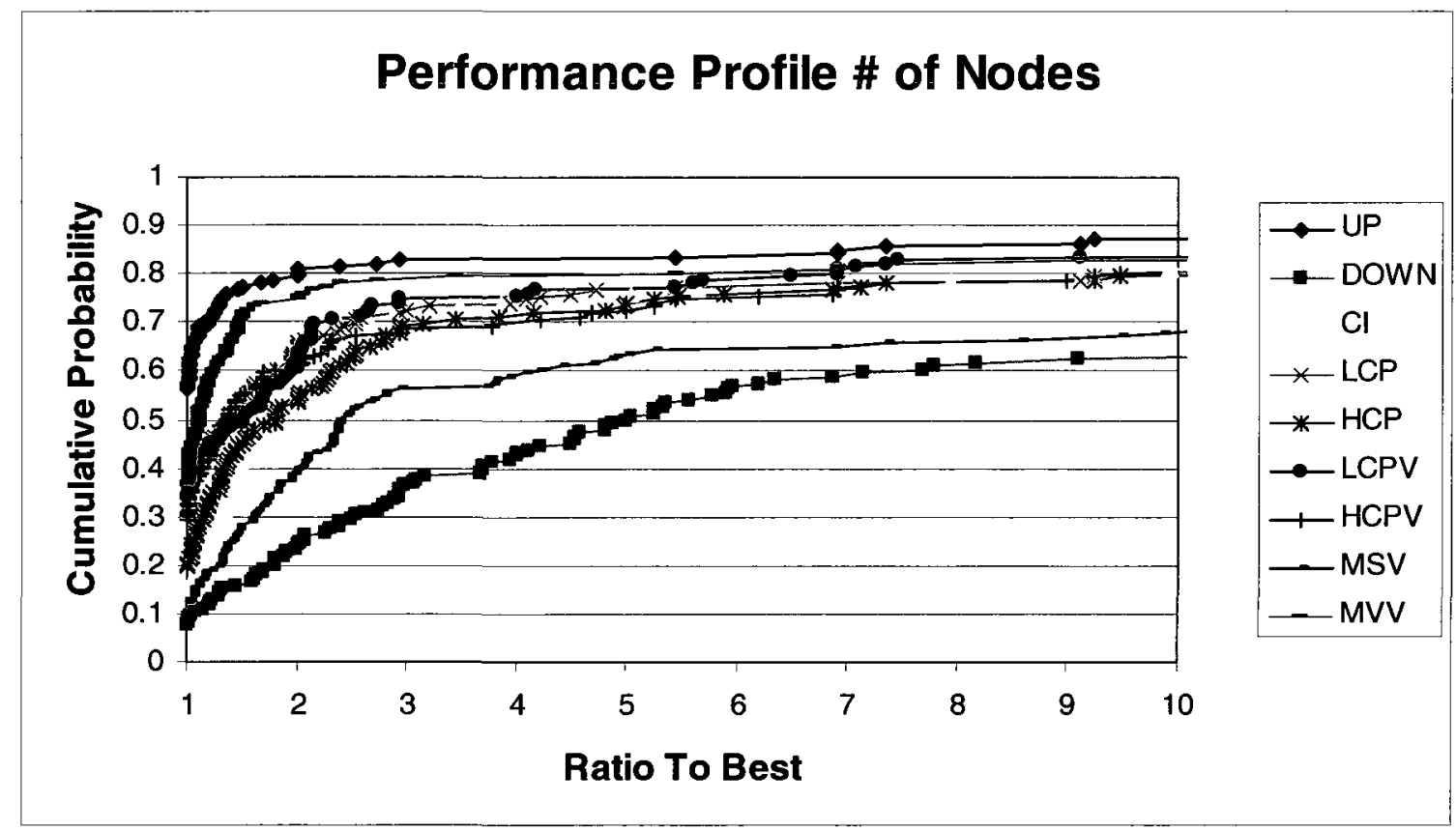

Figure 18. Experiment 1 performance profile for GLPK2 - number of nodes

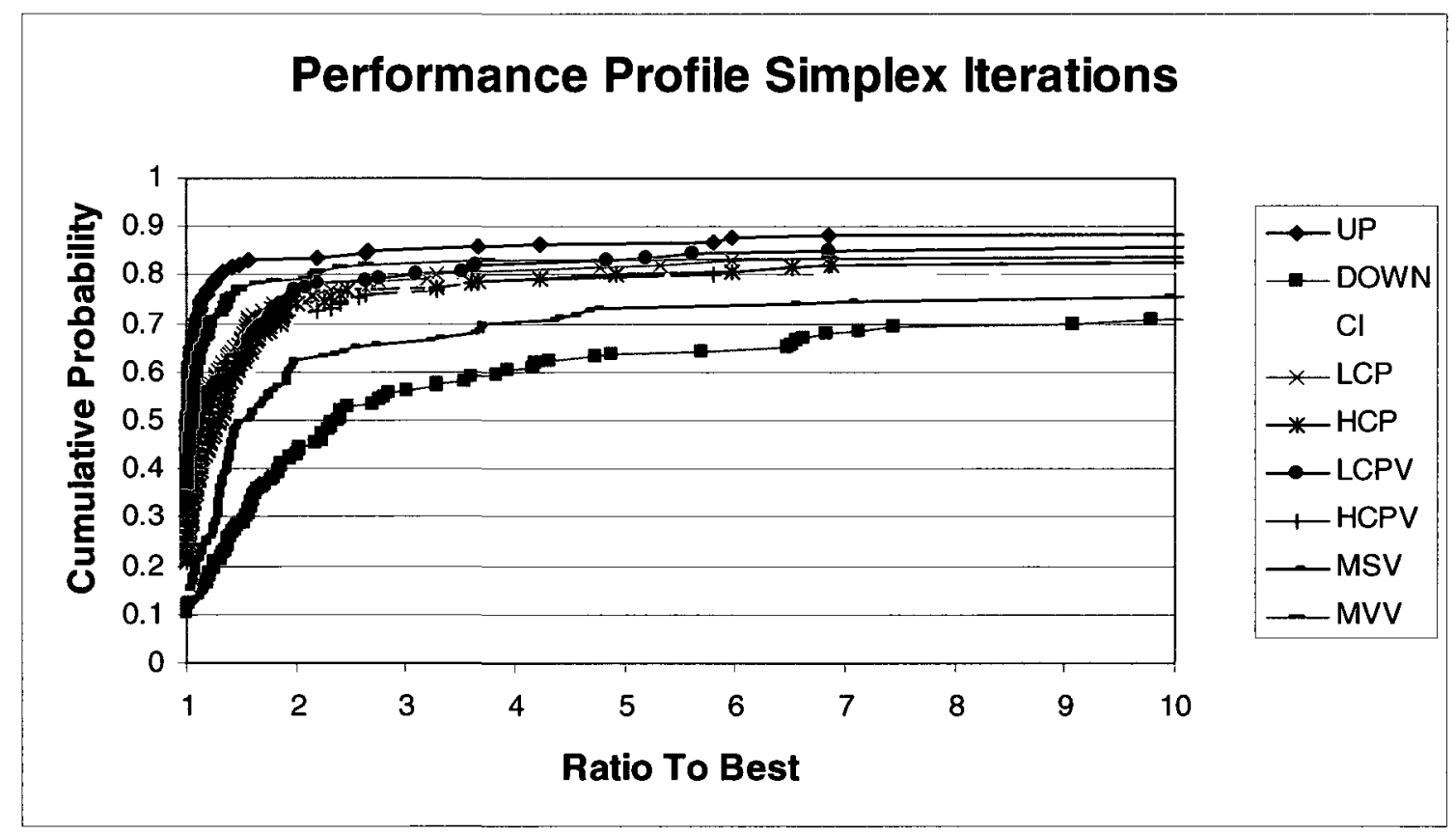

Figure 19. Experiment 1 performance profile for GLPK2 - simplex iterations 


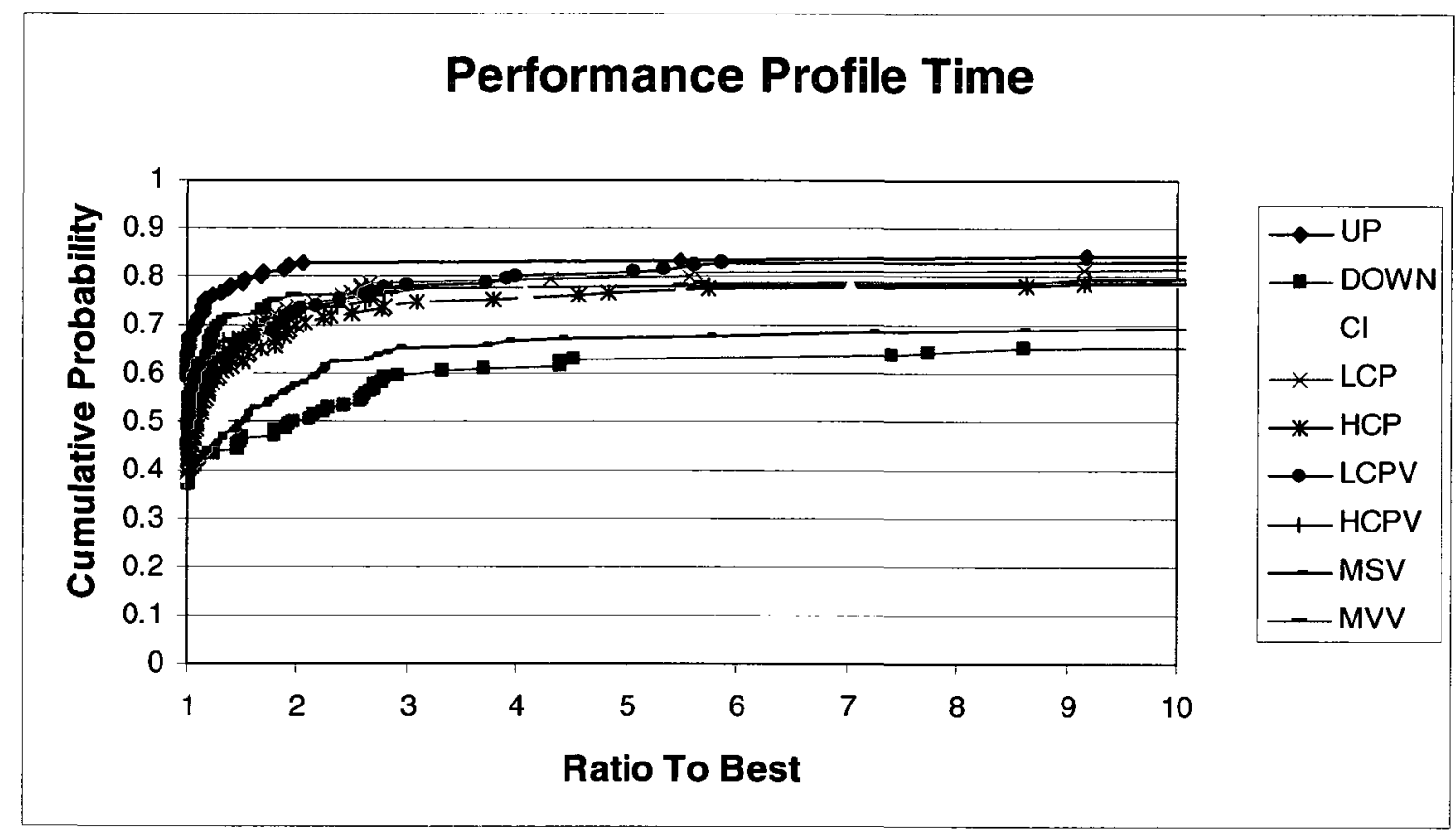

Figure 20. Experiment 1 performance profile for GLPK3 - computation time

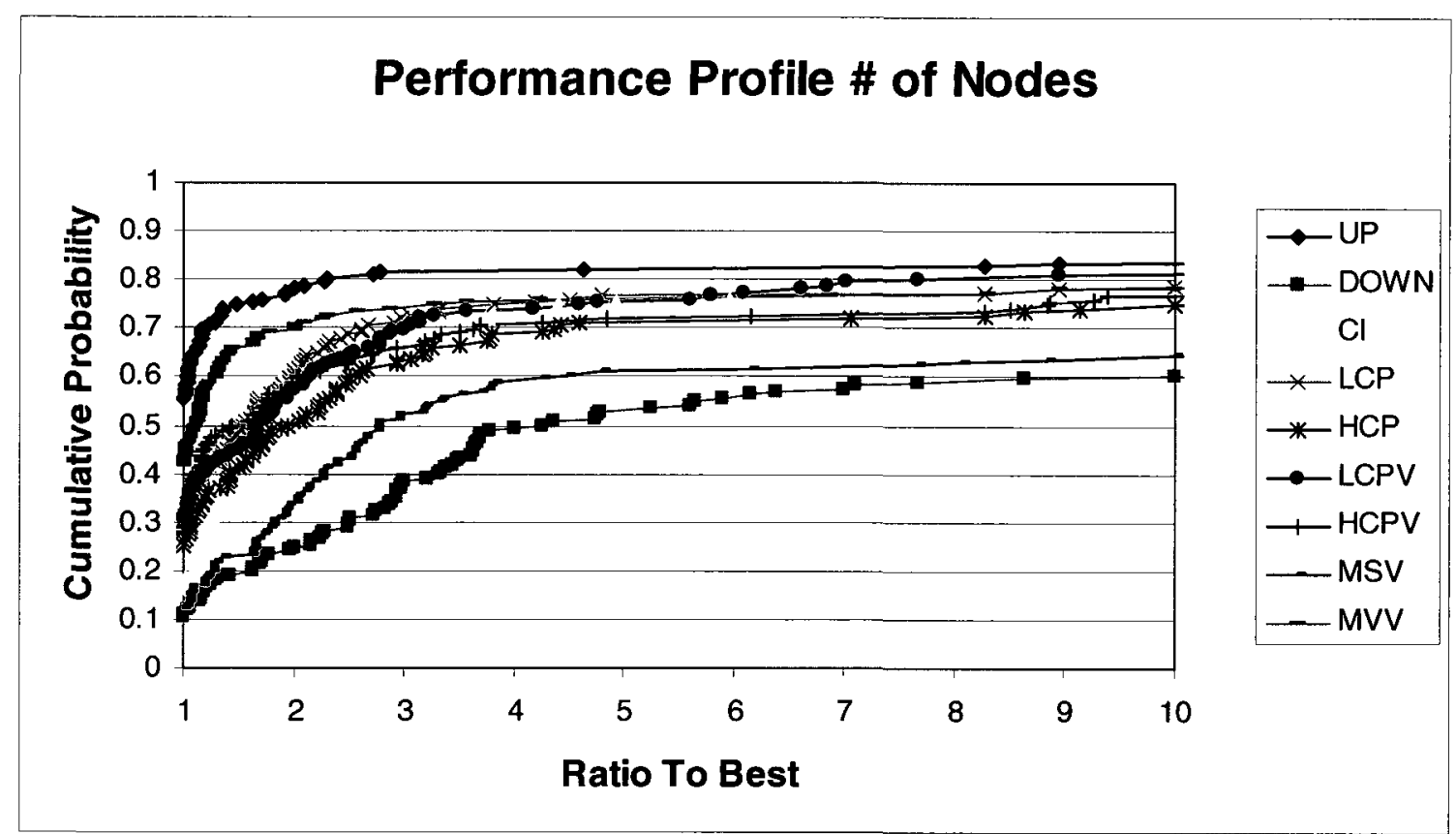

Figure 21. Experiment 1 performance profile for GLPK3 - number of nodes 


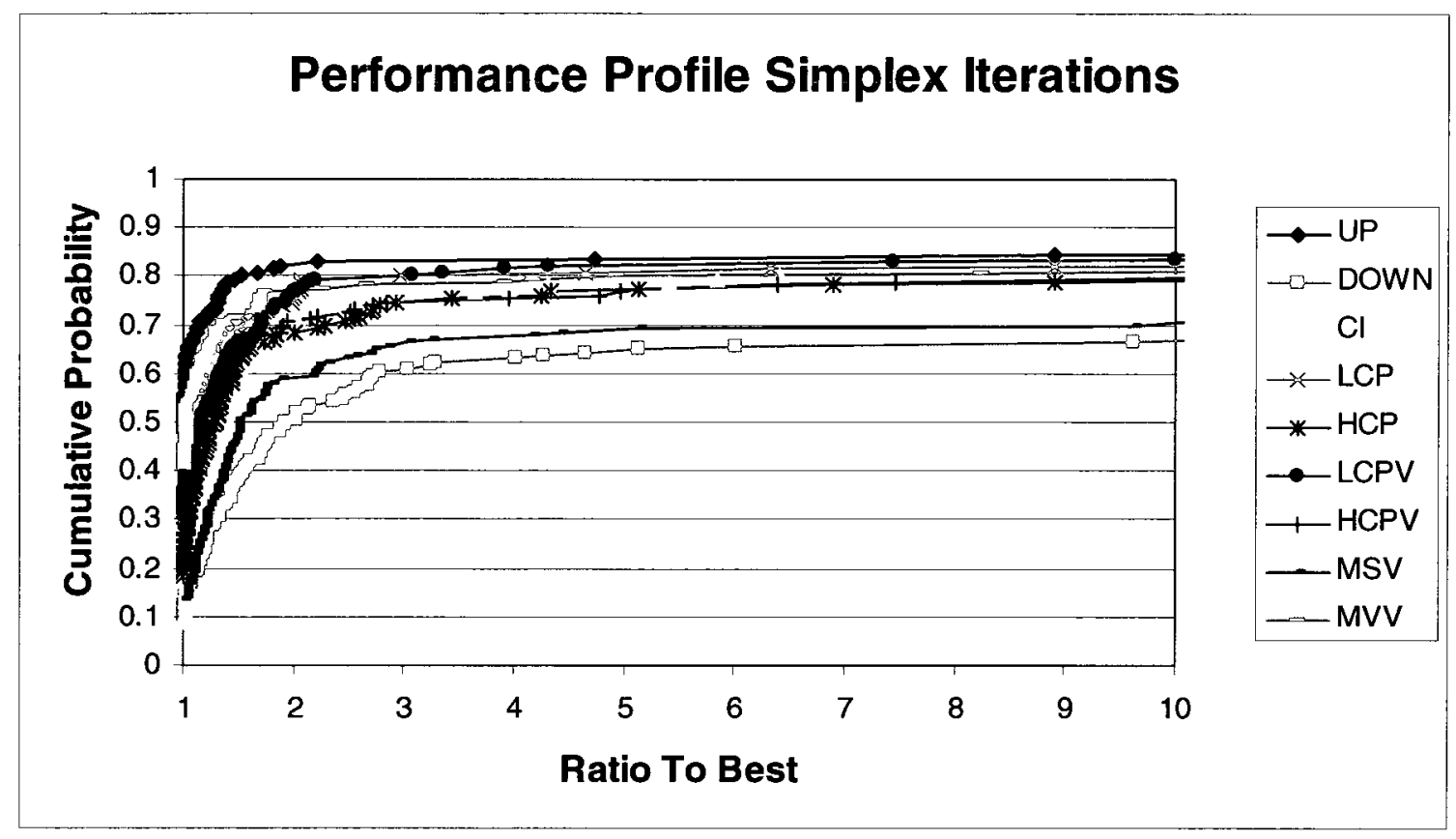

Figure 22. Experiment 1 performance profile for GLPK3 - simplex iterations

The next six figures have an additional branching direction method compared on the performance profiles called $D \& T$ or Driebeek and Tomlin. As mentioned previously, the Driebeek and Tomlin method has a branching direction routine built into the variable selection algorithm. Due to its dependence on the variable selection heuristic, D\&T could not be incorporated as a pure direction selection algorithm as the other nine direction methods are. Instead, the pure Driebeek and Tomlin method (variable and branching selection) is compared with the other nine direction selection methods using the Driebeek and Tomlin variable selection heuristic (GLPK4 and GLPKDEFAULT) while ignoring its built in direction selection. These results are consistent with the earlier findings; branching up is the superior method on all performance metrics. 


\section{Performance Profile Time}
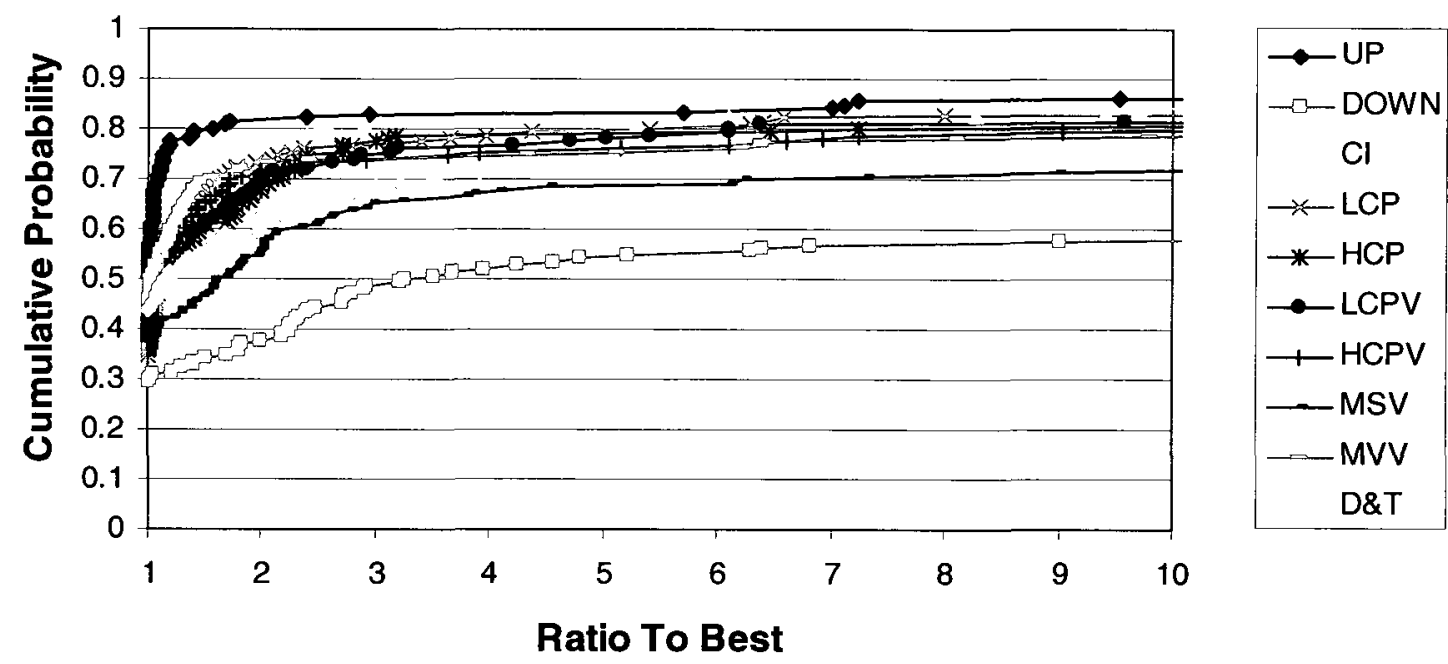

Figure 23. Experiment 1 performance profile for GLPK4 - computation time

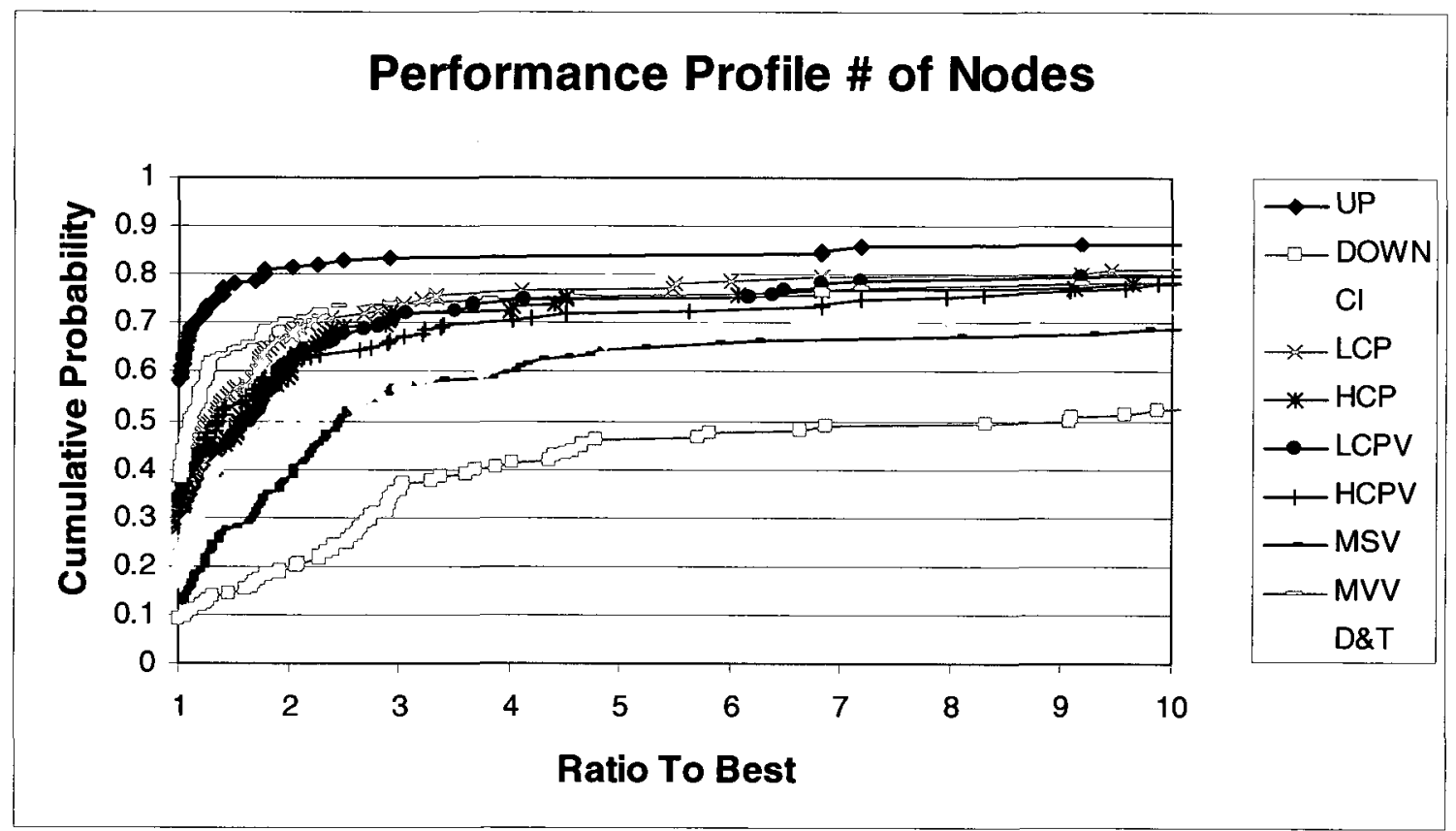

Figure 24. Experiment 1 performance profile for GLPK4 - number of nodes 


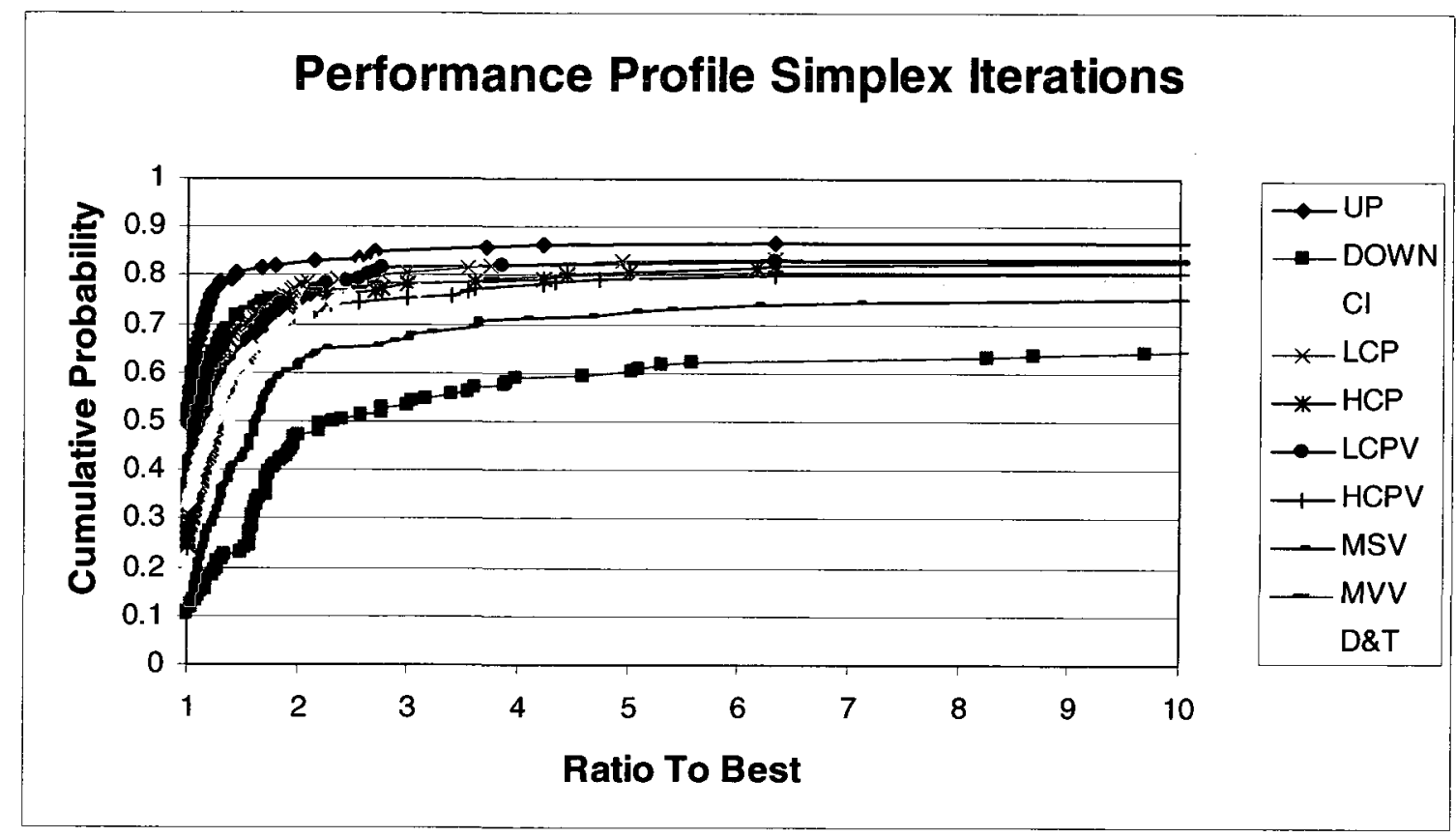

Figure 25. Experiment 1 performance profile for GLPK4 - simplex iterations

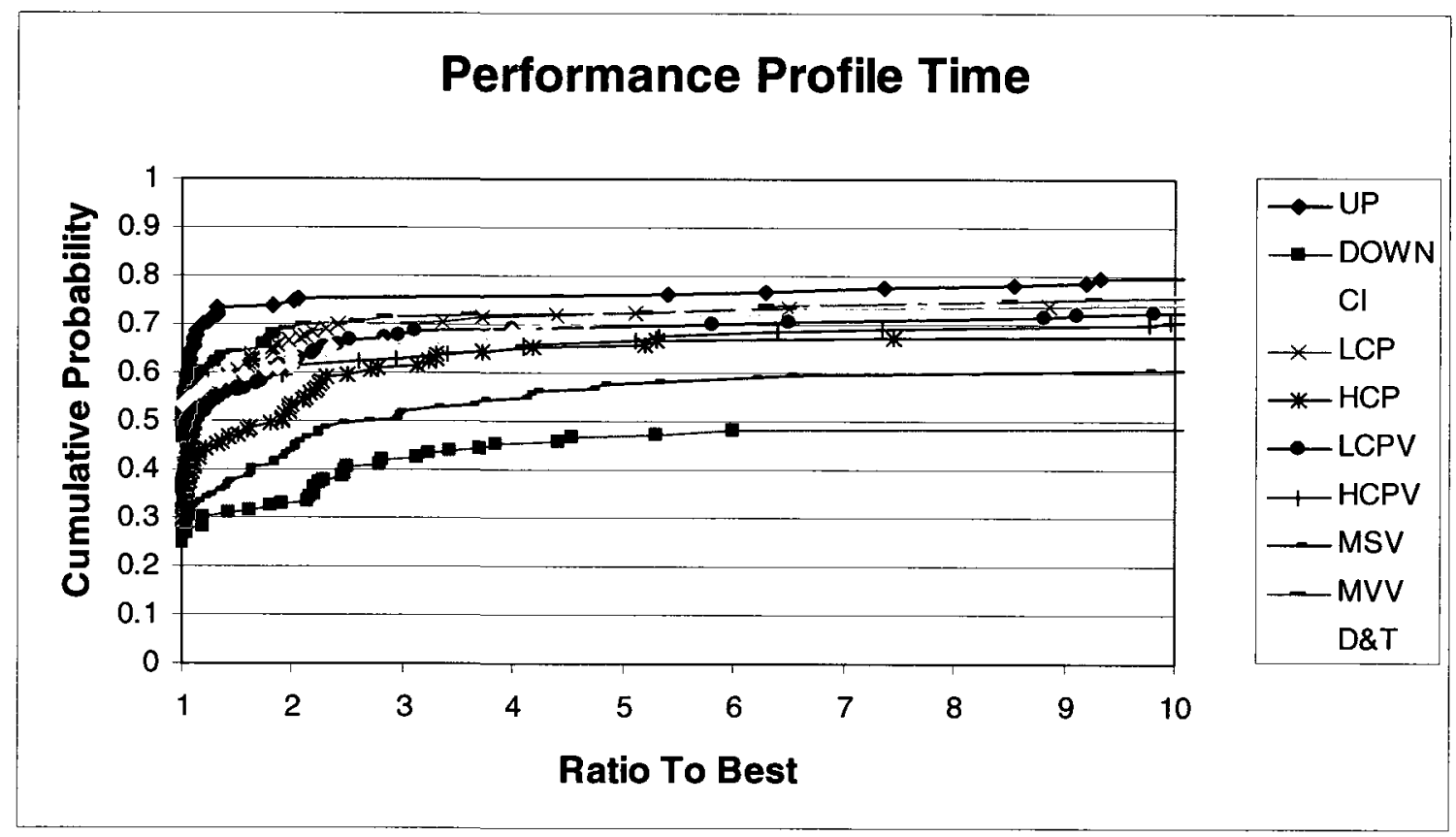

Figure 26. Experiment 1 performance profile for GLPKDEFAULT - computation time 


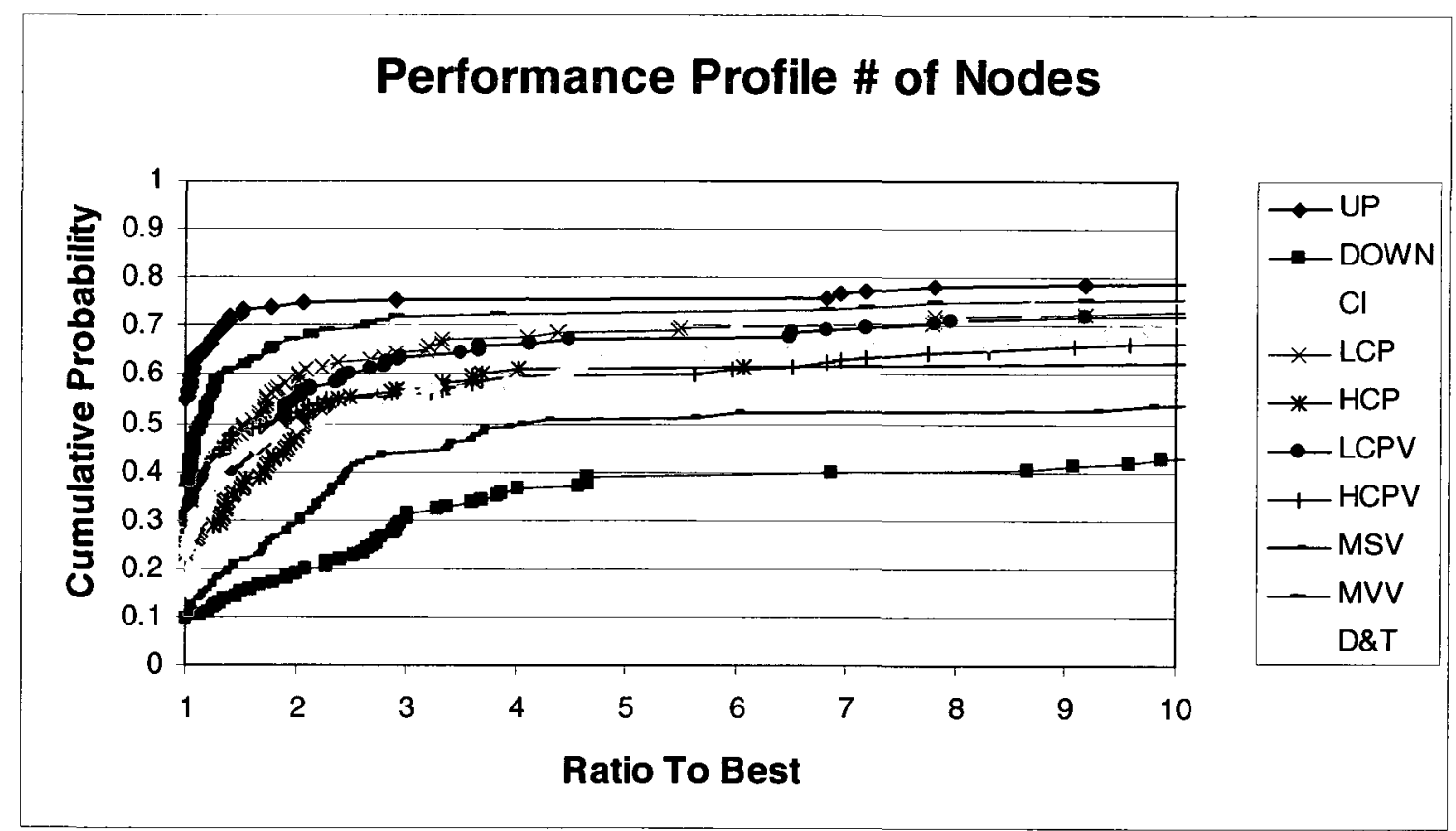

Figure 27. Experiment 1 performance profile for GLPKDEFAULT - number of nodes

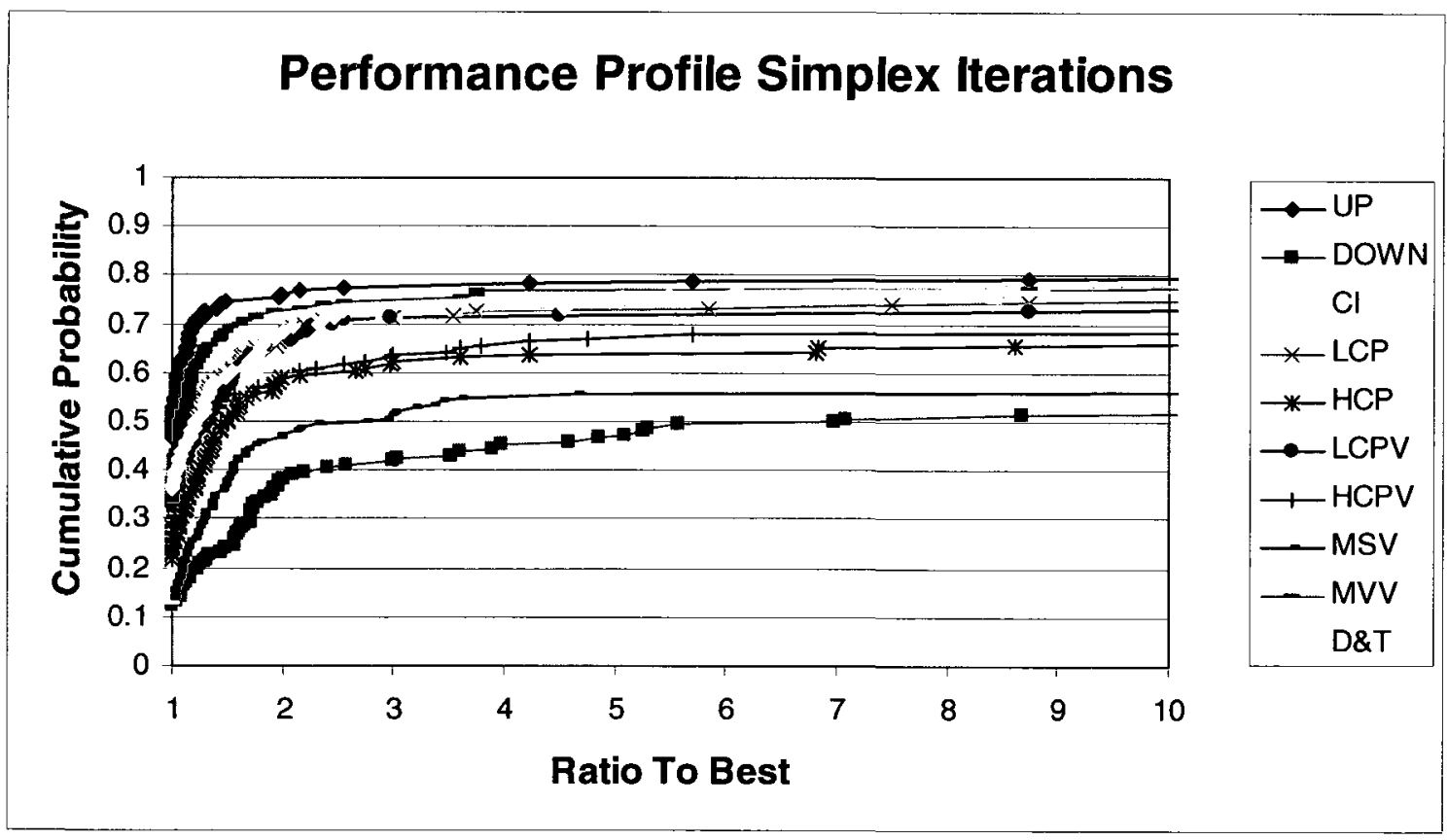

Figure 28. Experiment 1 performance profile for GLPKDEFAULT - simplex iterations 


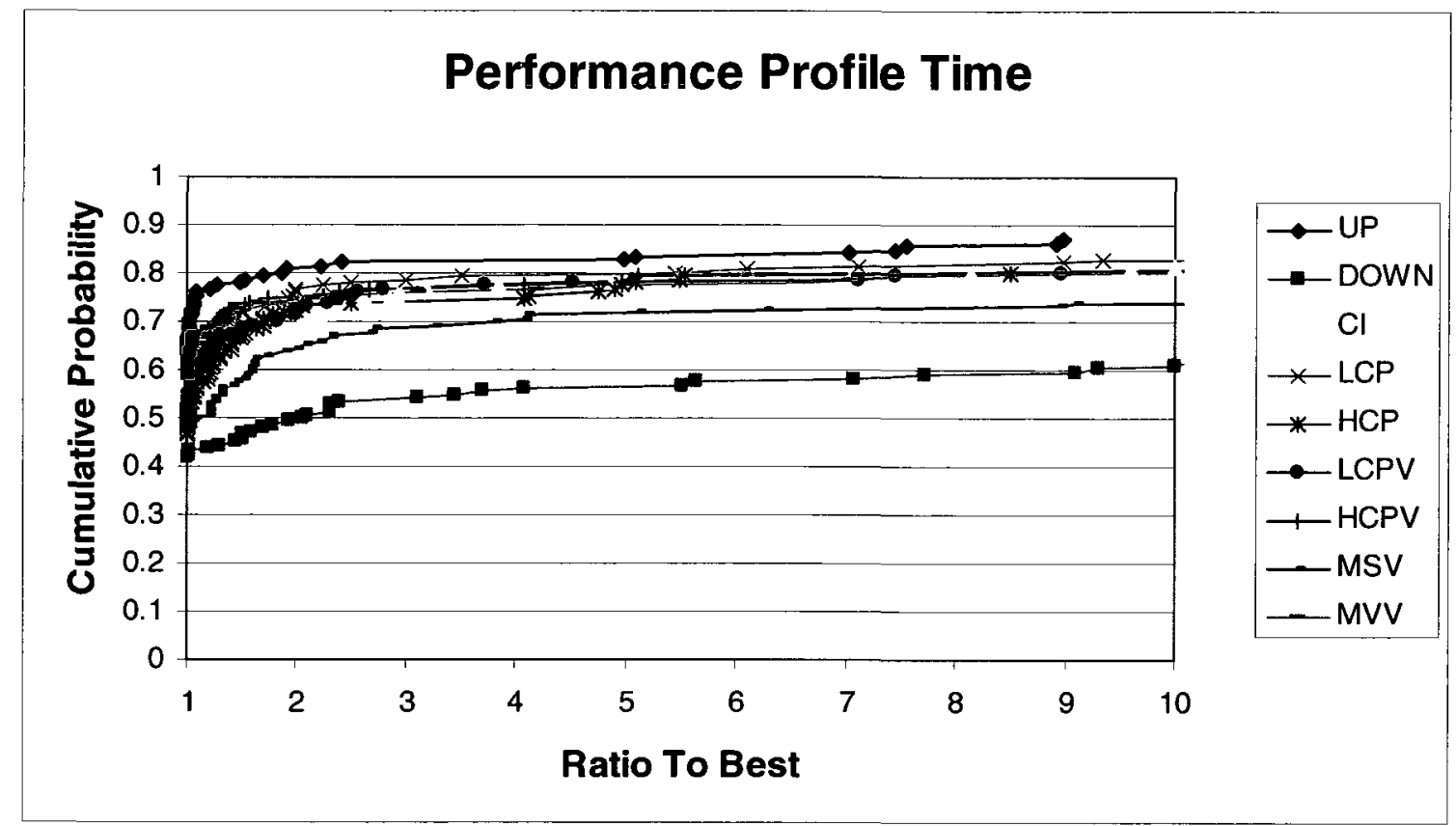

Figure 29. Experiment 1 performance profile for METHA - computation time

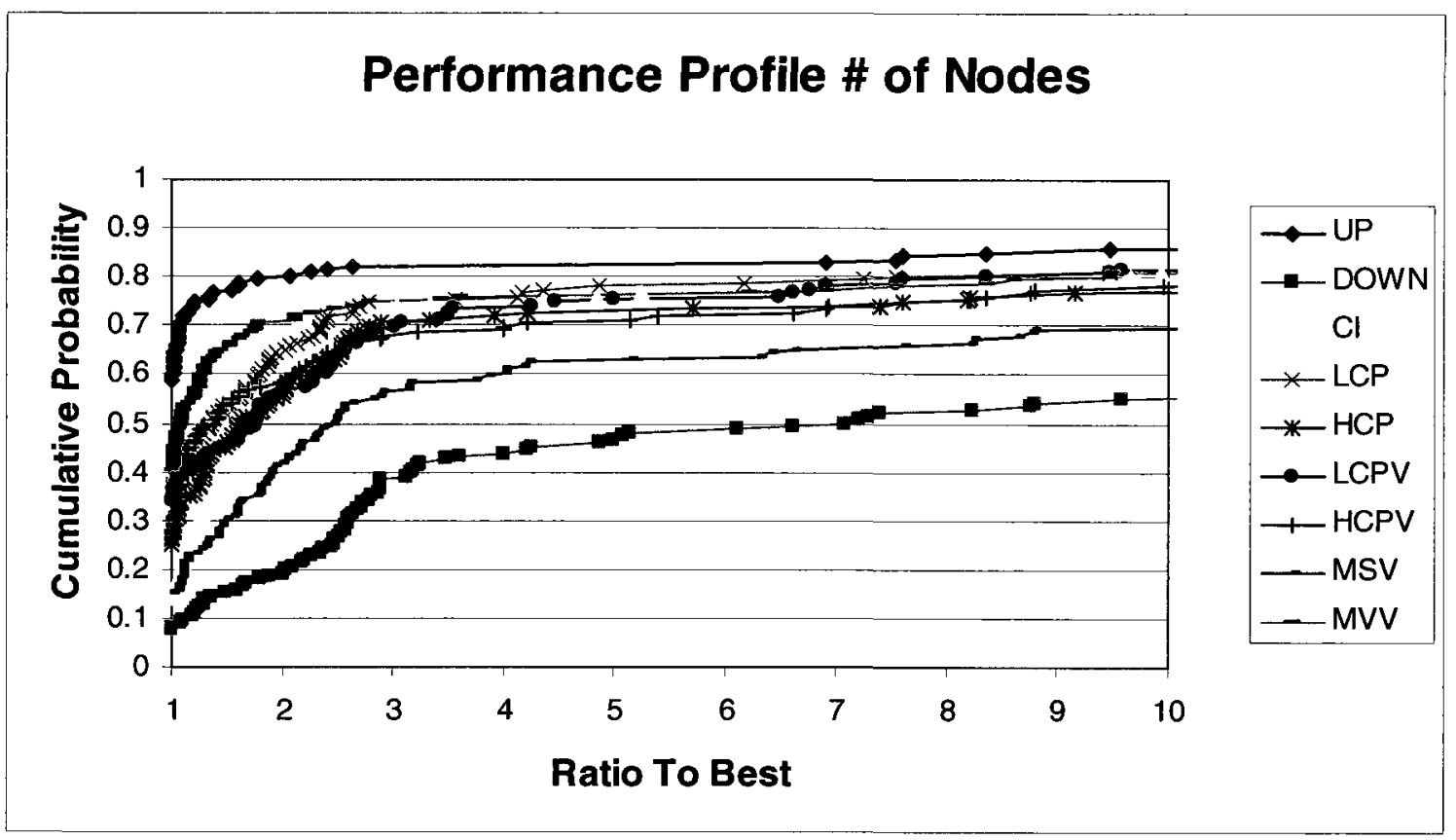

Figure 30. Experiment 1 performance profile for METHA - number of nodes 


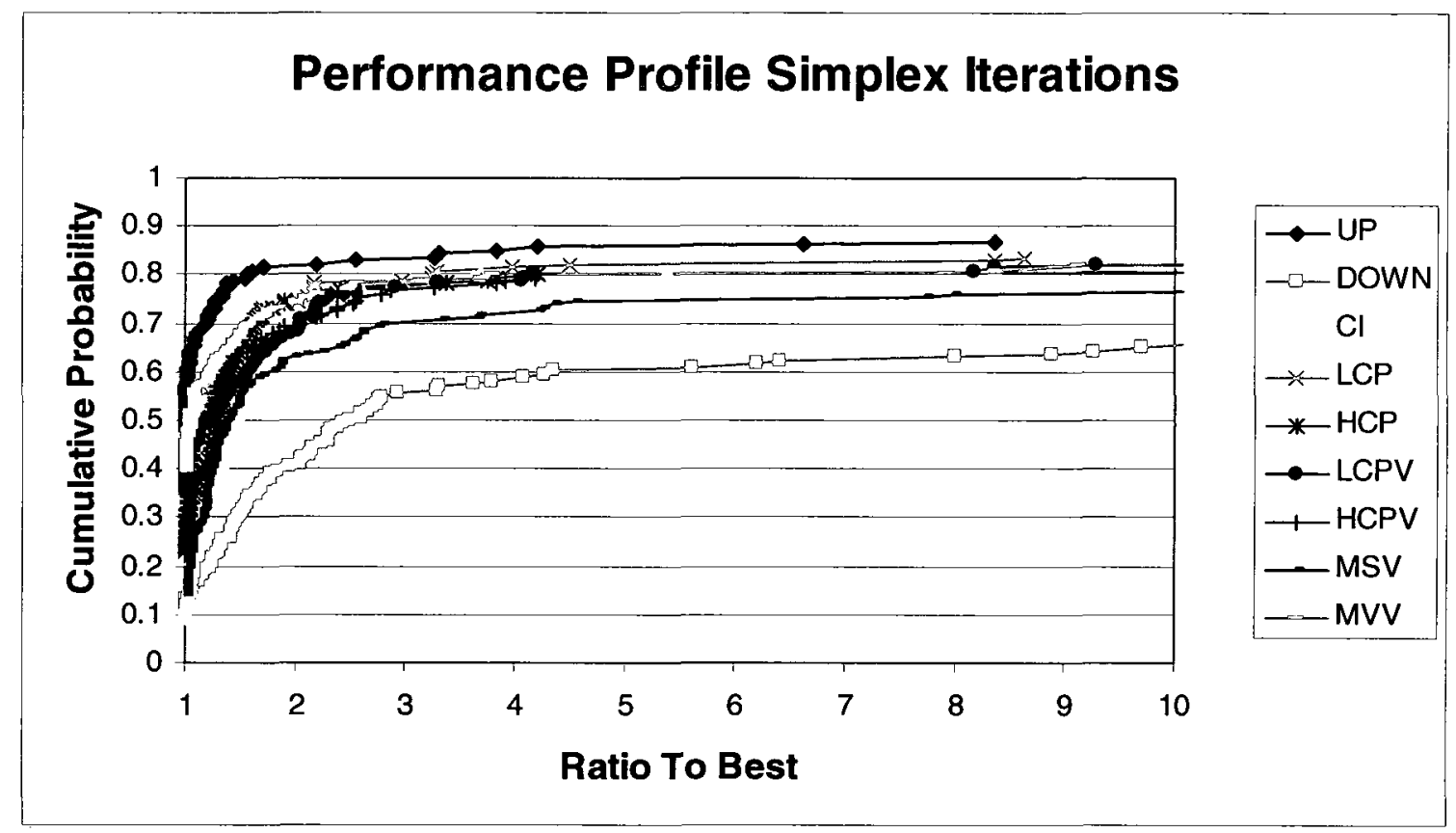

Figure 31. Experiment 1 performance profile for METHA - simplex iterations

From the above graphs, it is clear that UP is the winner on all three performance metrics. It has the most wins (reaches the highest point on the y-axis of all the methods), is the most robust, and there is a sizable gap between it and the closest competing branching direction selection method. The second closest method is MVV, branching in the violated direction.

The next three graphs are summary plots of the above eighteen graphs. They are created by transferring all the points to a single graph and connecting those that use the same branching direction heuristic. For example, Figure 32 displays the overall results for Experiment 1 in terms of time; it takes all of the points from the previous six computation time graphs and connects those that use the same branching direction heuristic. The graph itself shows how each branching direction heuristic fared over all of the MIP/variable selection/node selection combinations. The conclusions from these summary graphs are that regardless of the variable selection and node selection scheme used, UP is the best 
branching direction heuristic. MVV is the second closest branching direction heuristic. In addition, DOWN is the worst branching direction heuristic. Figure 33 and Figure 34 show the overall results for number of nodes, and simplex iterations respectively.

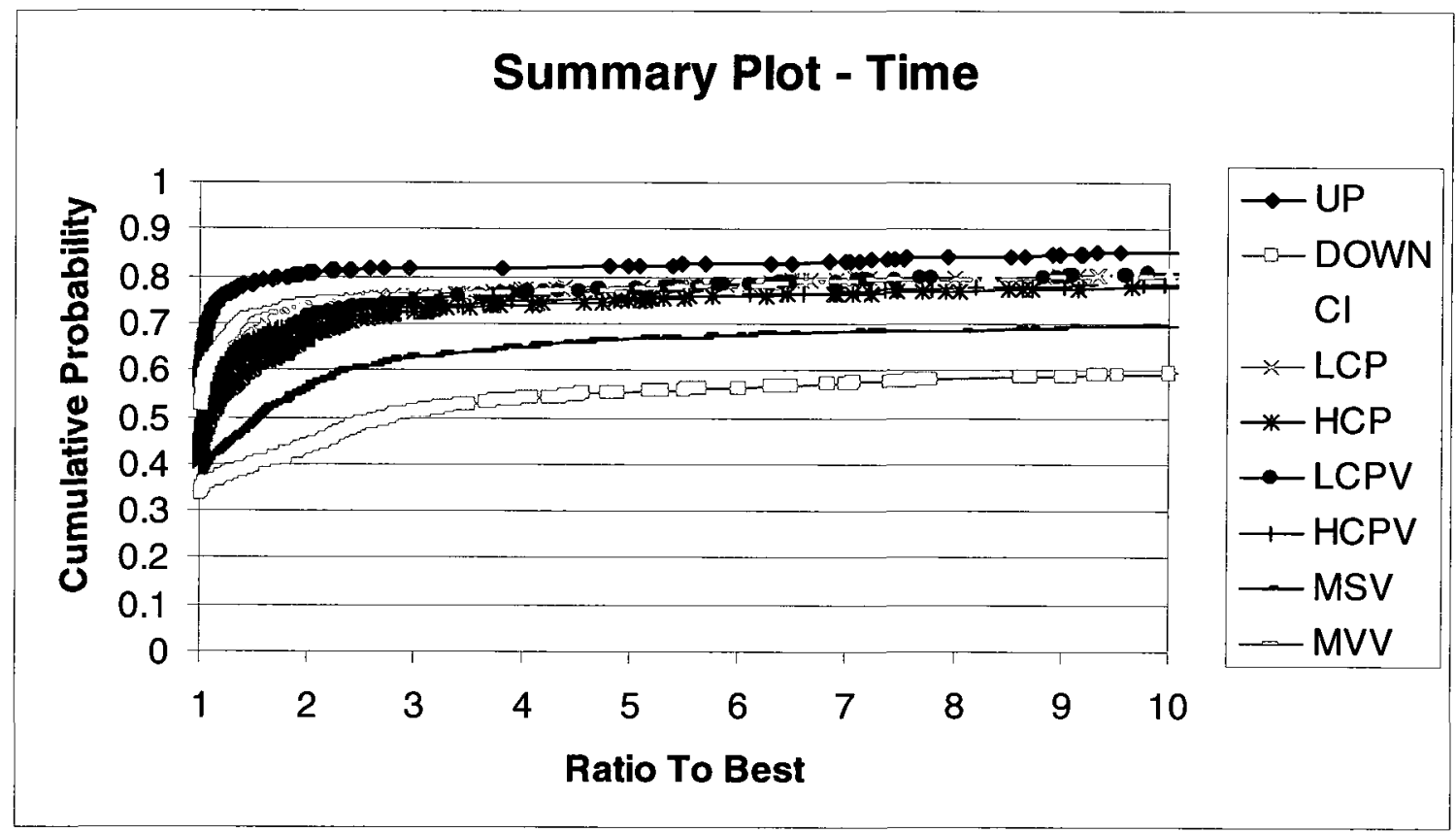

Figure 32. Experiment 1 summary plot for overall data - computation time

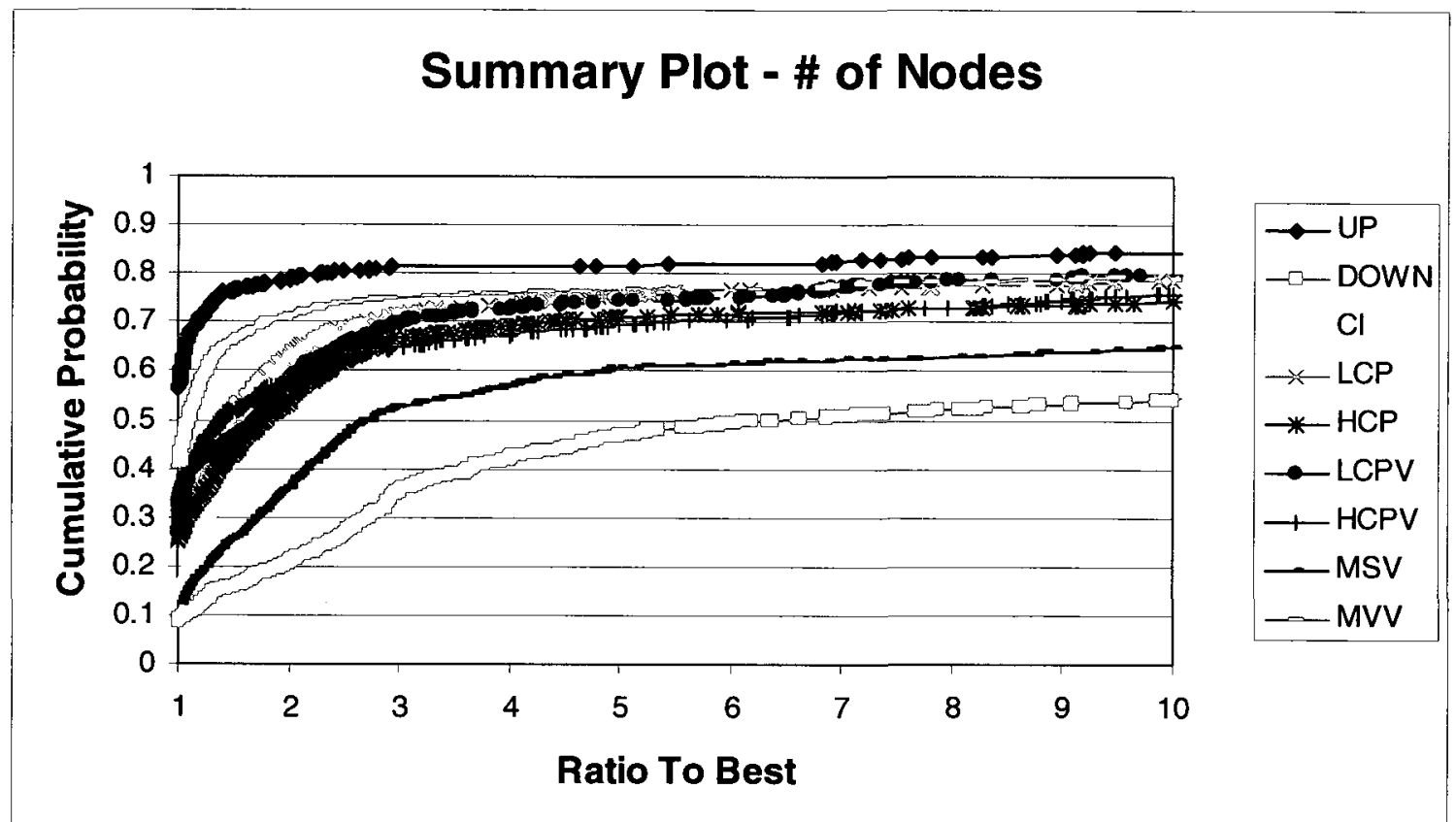

Figure 33. Experiment 1 summary plot for overall data - number of nodes 


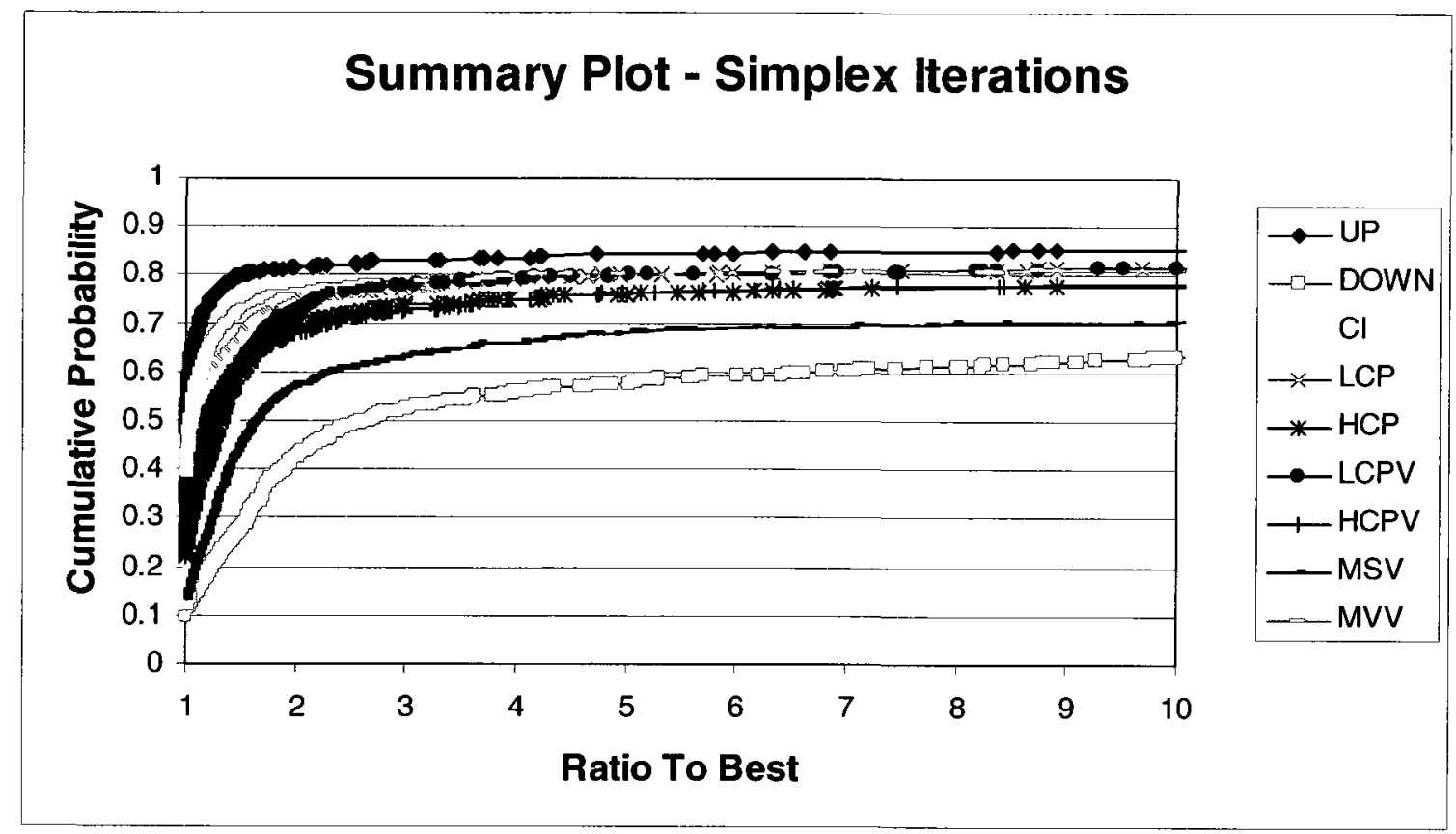

Figure 34. Experiment 1 summary plot for overall data - simplex iterations

The next six figures separate the data by equality-free and equality-containing

MIPs. The first three summary plots display the data from solving the equality-containing MIPs, while Figure 38, Figure 39, and Figure 40 display the data from the equality-free MIPs.

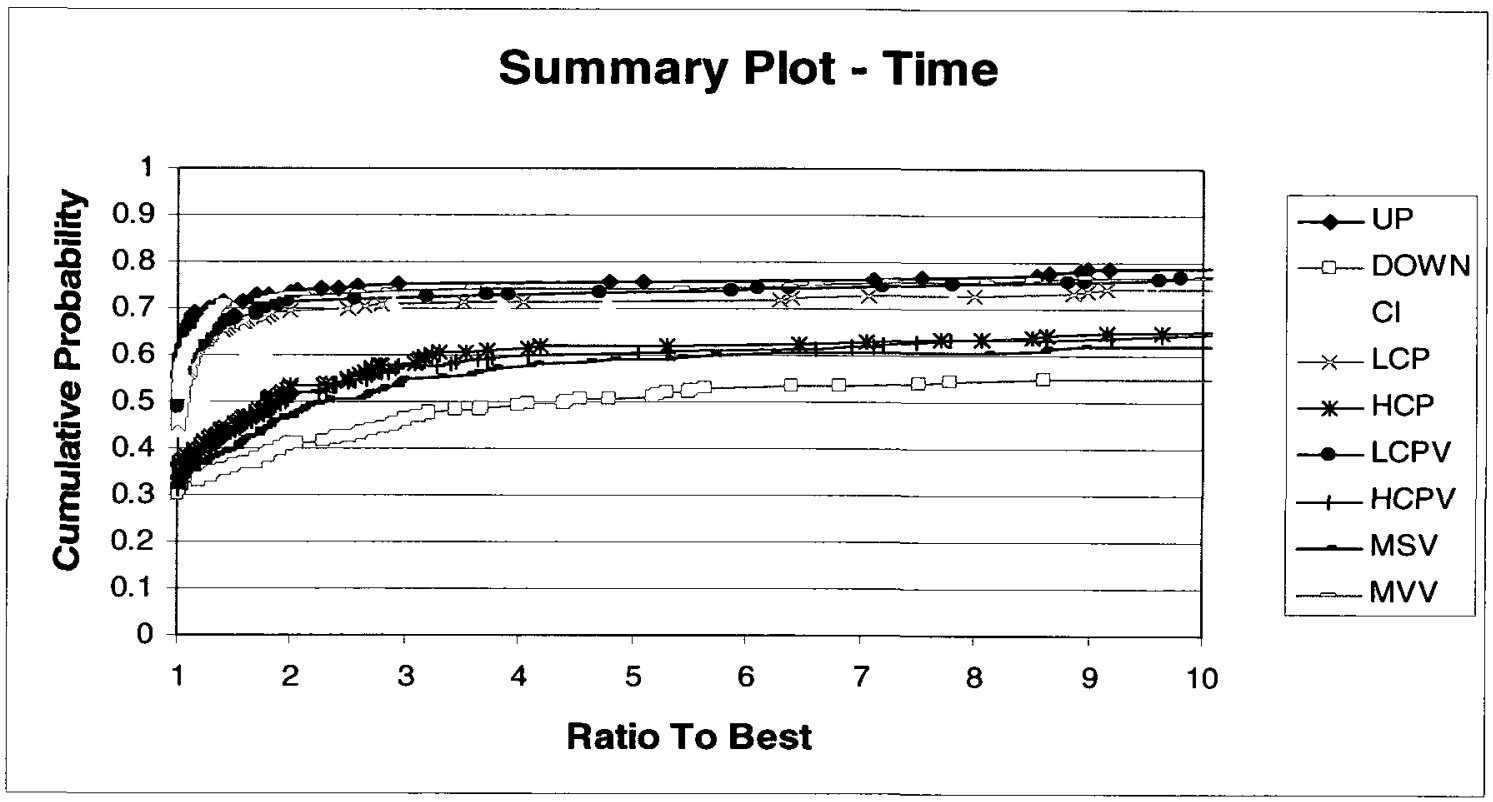

Figure 35. Experiment 1 summary plot for equality-containing - computation time 


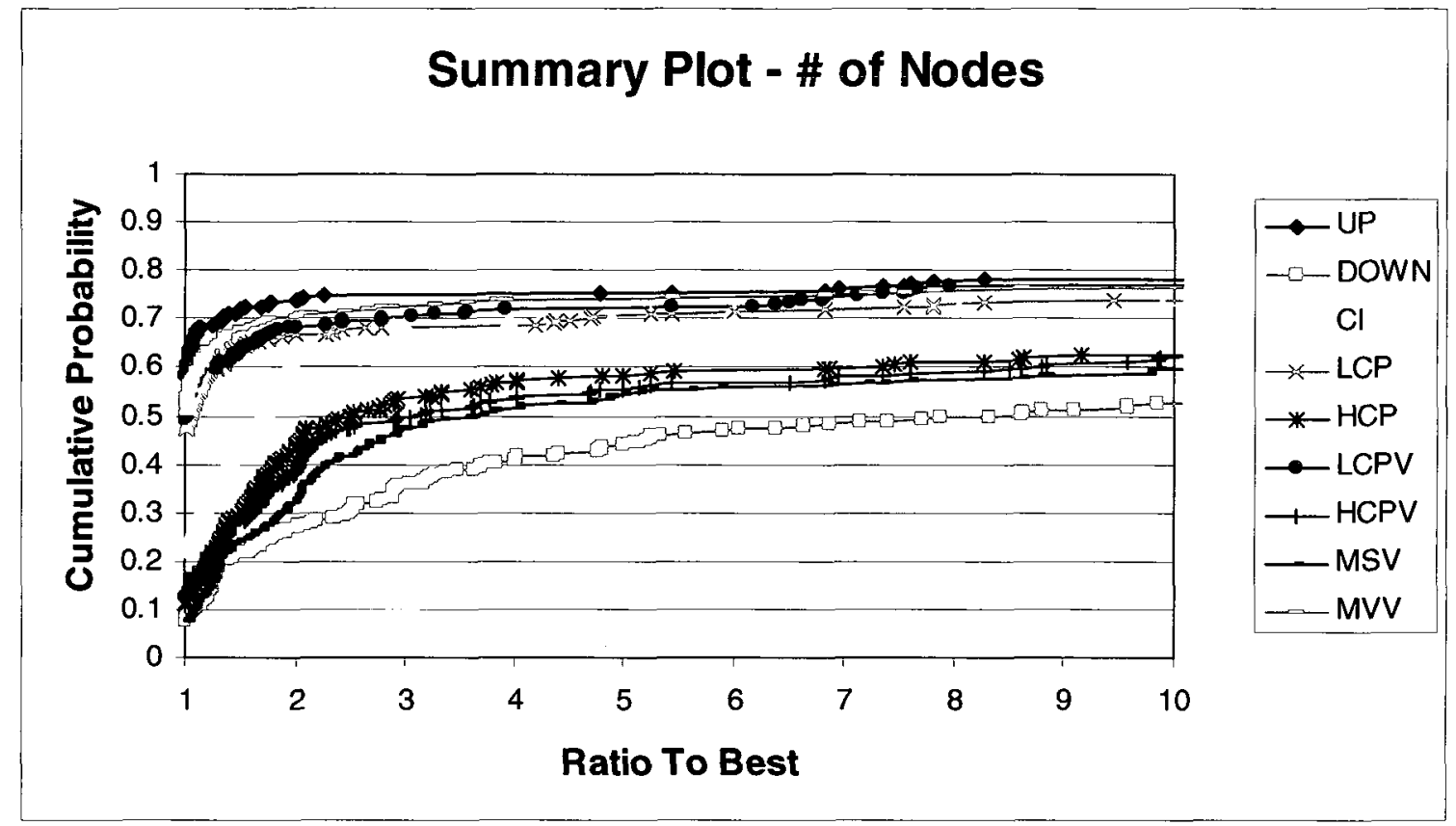

Figure 36. Experiment 1 summary plot for equality-containing - number of nodes

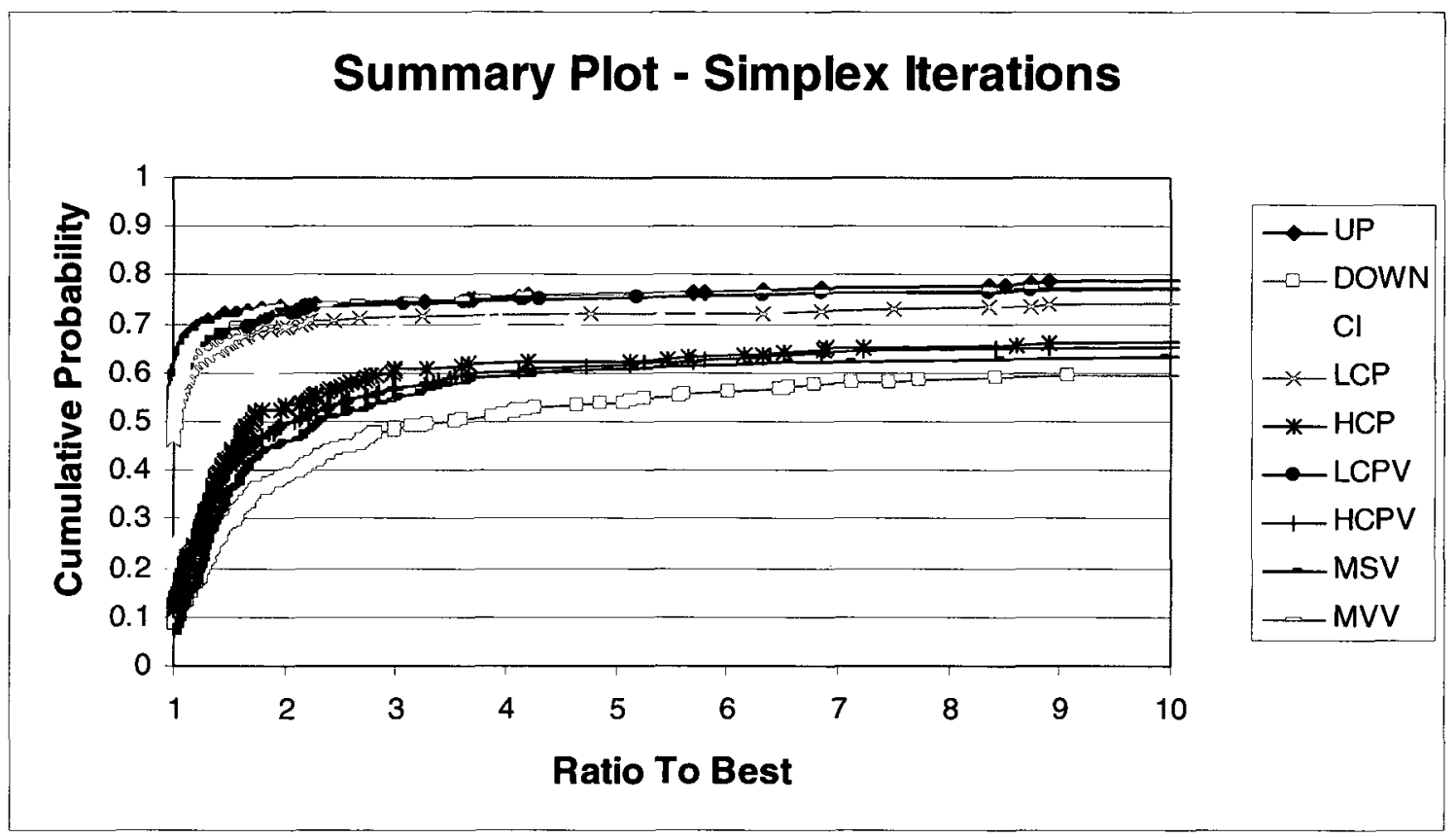

Figure 37. Experiment 1 summary plot for equality-containing - simplex iterations 


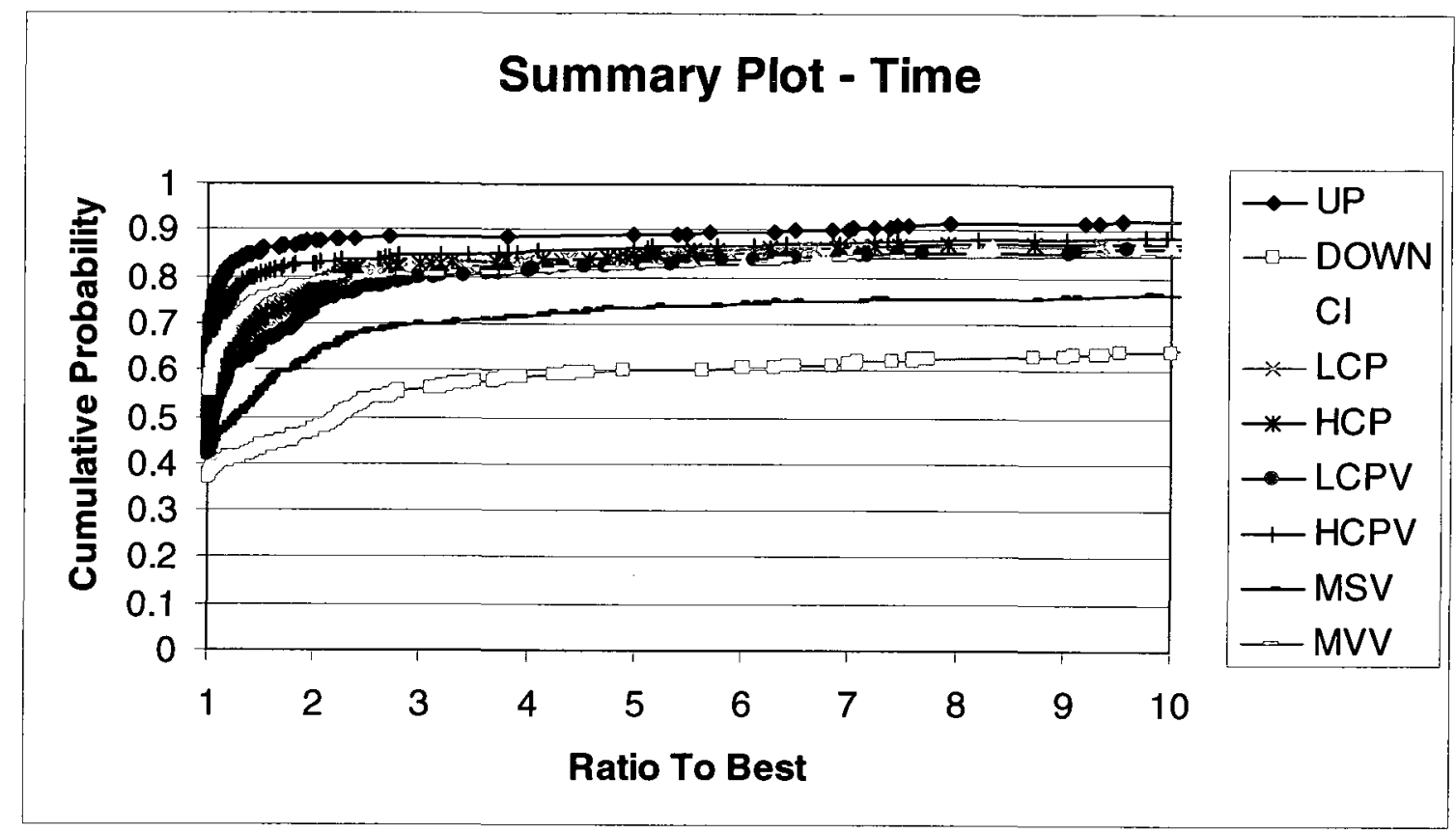

Figure 38. Experiment 1 summary plot for equality-free - computation time

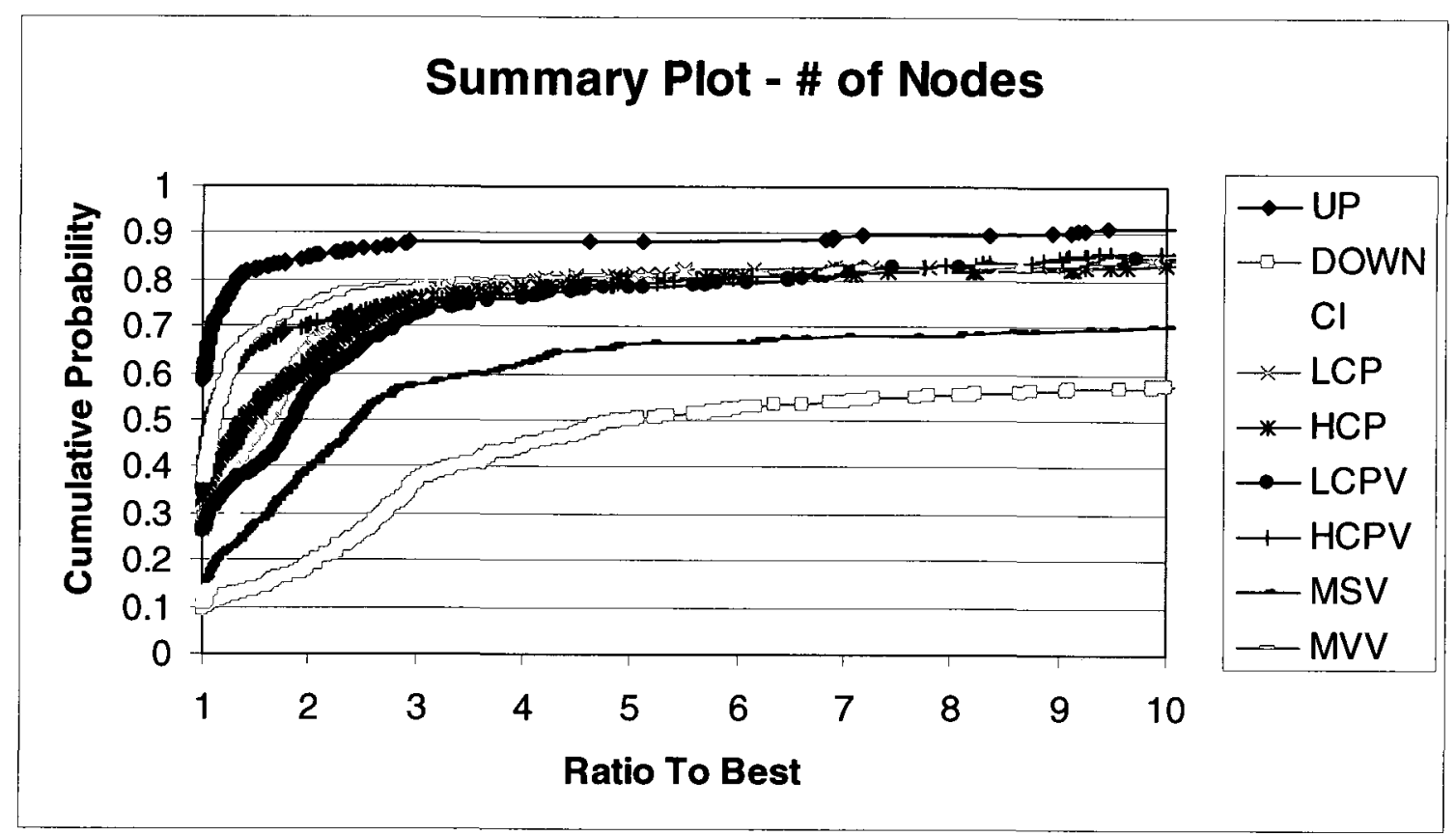

Figure 39. Experiment 1 summary plot for equality-free - number of nodes 


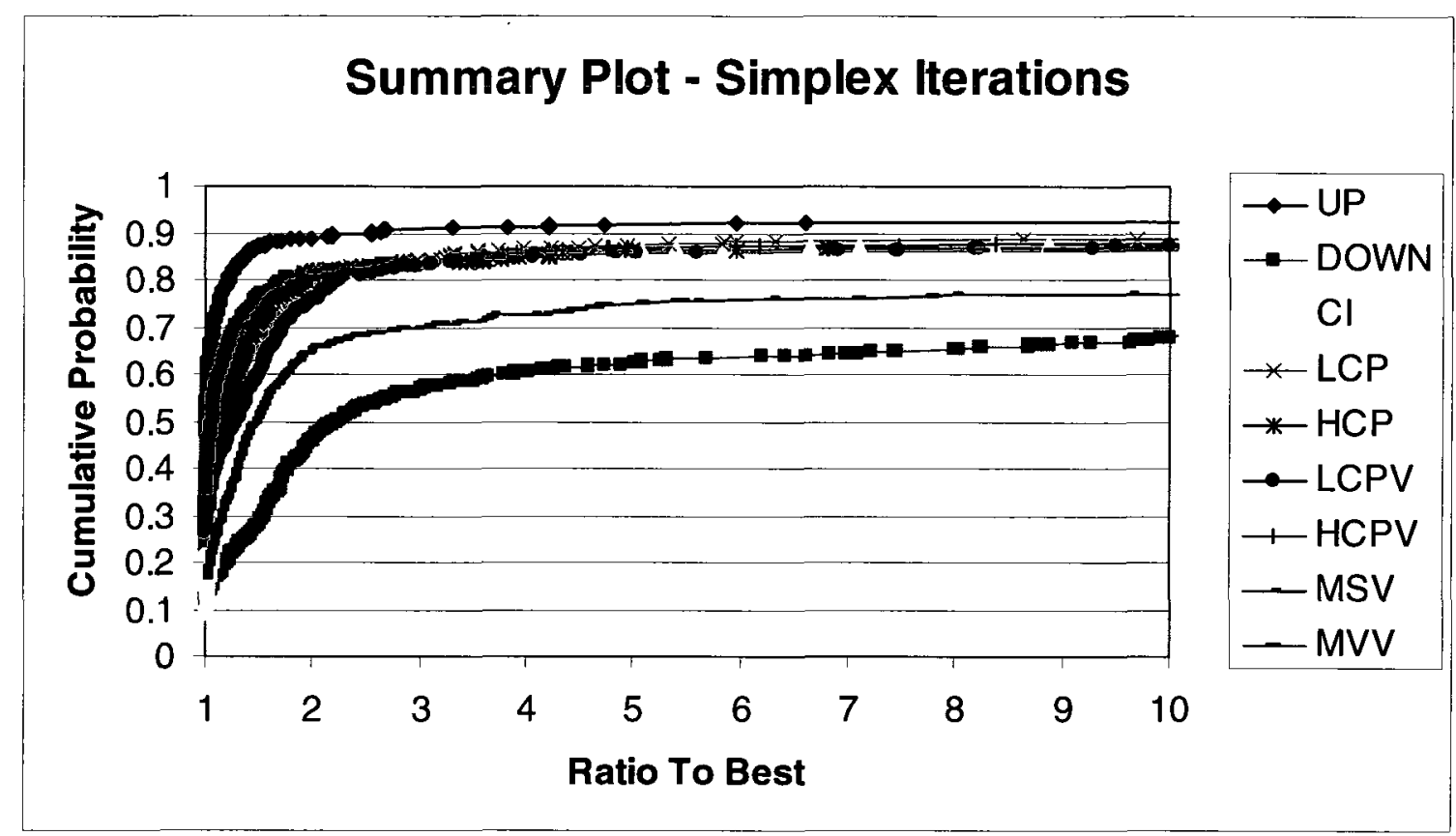

Figure 40. Experiment 1 summary plot for equality-free - simplex iterations

A few differences are noted when comparing the set of equality-free MIPs to the equality-containing MIPs (regardless of the variable and node selection methods). First of all, the branching direction heuristics are significantly less robust when solving the equality-containing MIPs. At best, they obtain an $82 \%$ success rate on the equalitycontaining MIPs; however they solve $93 \%$ of the equality-free MIPs. In addition, on the equality-containing MIPs, MVV virtually equals the performance of UP in the performance metrics, whereas there is a gap between the two methods in the equality-free MIPs.

From Experiment 1 there are a few overall observations and conclusions. UP is always the best branching direction heuristic on all combinations tested. Similarly, DOWN is always the worst branching direction heuristic, and MVV is always the second best branching direction heuristic. In addition, when looking at the types of constraints 
present in a MIP, UP has close competition for the best branching direction heuristic only on MIPs containing equality constraints. 


\section{Experiment 2: Simultaneous Selection of Branching Variable and Direction}

Experiment 2 looks at various algorithms that simultaneously select the branching variable and direction. Experiment 2 is broken into four sub-sets of experimental results:

- The eight probability methods that use cumulative and point probabilities.

- The eight probability methods that use multiplied probabilities.

- Differences between equality-free and equality-containing MIPs.

- Hybrid methods.

Experiment 2 was broken into these four subsets to allow for quick experimental runs that would determine the top methods of the original sixteen probability algorithms. To reiterate, the differences between Experiment 1 and 2 are:

- Experiment 1: Given the branching variable by some method, which direction heuristic is best?

- Experiment 2: Simultaneous variable and direction selection.

\subsection{Cumulative and Point Probability Methods}

\subsubsection{Goal of Experiment}

This experiment implements the eight cumulative and point probability variable and direction selection algorithms listed in Table 3 and Table 4. These algorithms are just compared to GLPK1, GLPK2, GLPK3, and GLPK4 (Table 7) to identify the top cumulative and point probability methods that will move on to further testing. Each of the branching variable and direction selection methods are tested on the ninety-five equalityfree MIPs because they run faster than the equality-containing MIPs. 


\subsubsection{Experimental Results}

Each performance profile below specifies which metric (time, number of nodes, or simplex iterations) it is measuring. From these results only VDS-LCP, which selects the branching variable and direction with the lowest cumulative probability, is competitive with the best of the GLPK methods. VDS-LCP is the only method from the eight to move on to further testing in Section 8.3.

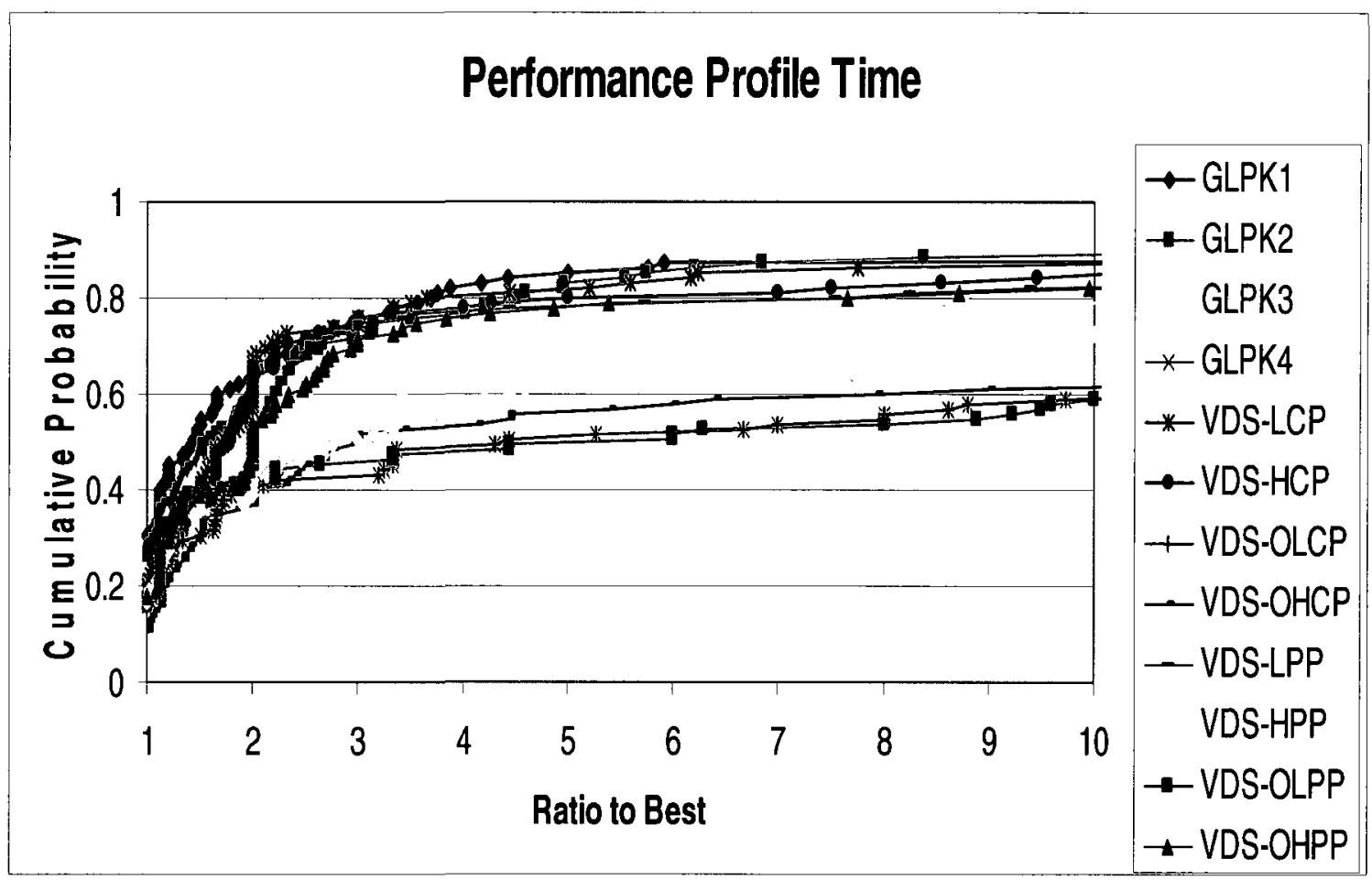

Figure 41. Experiment 2 performance profile for cumulative and point probability methods on equality-free MIPs - computation time 


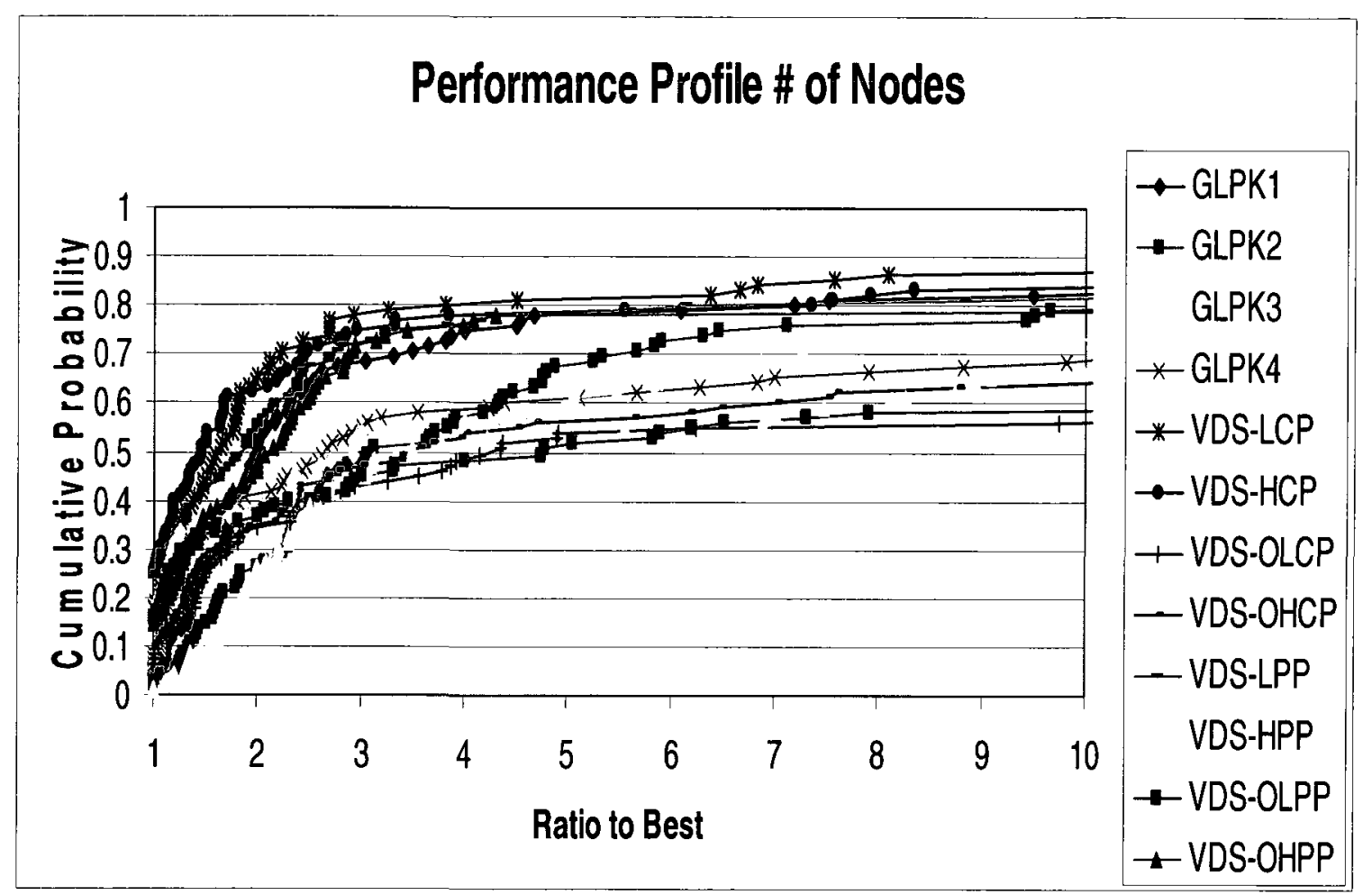

Figure 42. Experiment 2 performance profile for cumulative and point probability methods on equality-free MIPs - number of nodes

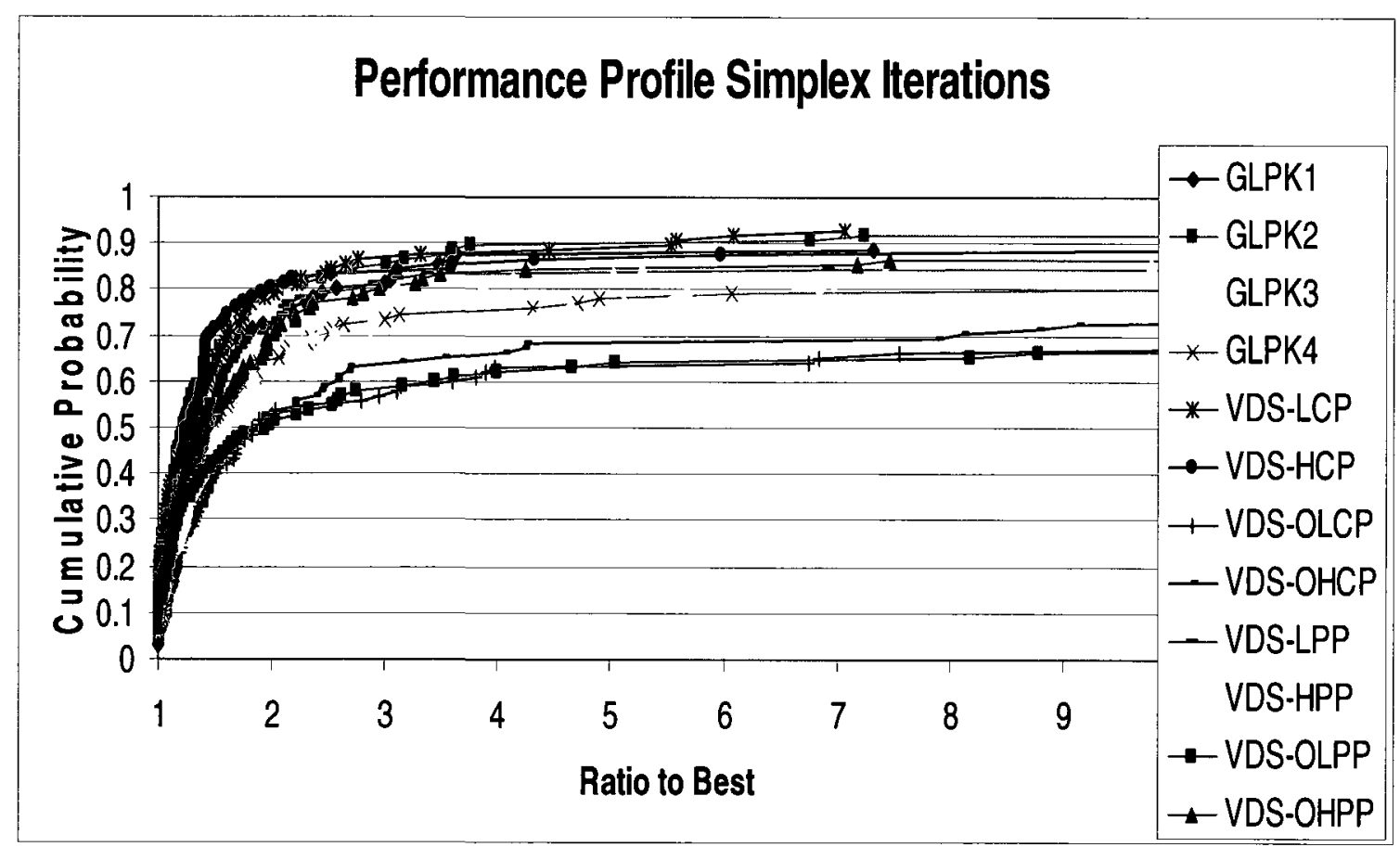

Figure 43. Experiment 2 performance profile for cumulative and point probability methods on equality-free MIPs - simplex iterations 


\subsection{Multiplied Probability Methods}

\subsubsection{Goal of Experiment}

This experiment implements the eight multiplied probability variable and direction selection algorithms listed in Table 5. These algorithms are just compared to GLPK1, GLPK2, GLPK3, and GLPK4 (Table 7) to identify any competitive multiplied probability methods that will move on to further testing. Each of the branching variable and direction selection methods are tested on the ninety-five equality-free MIPs because they run faster than the equality-containing MIPs.

\subsubsection{Experimental Results}

Each performance profile below specifies which metric (time, number of nodes, or simplex iterations) it is measuring. From these results none of the eight multiplied probability methods are competitive with the best of the GLPK methods. No further testing was performed on these methods.

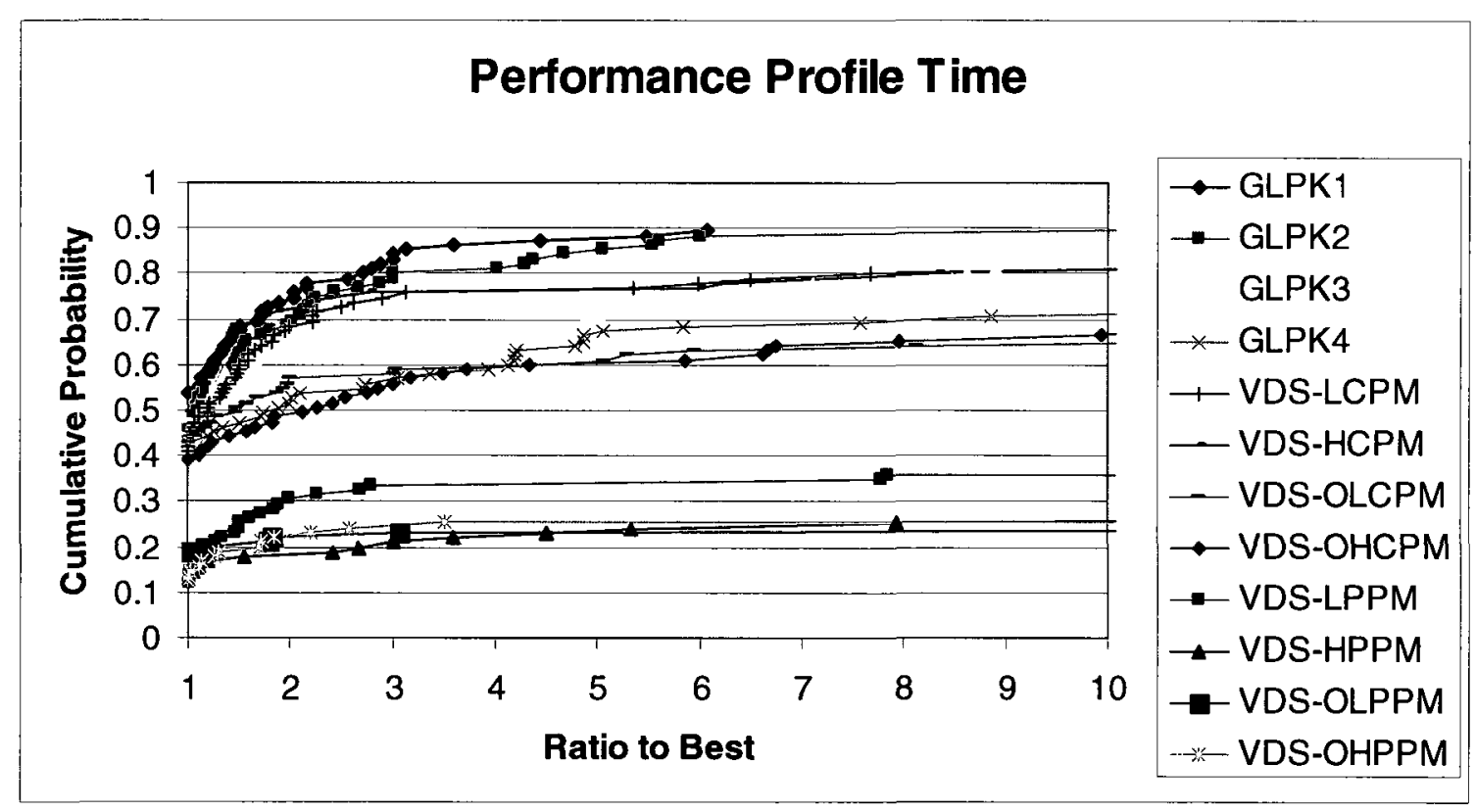

Figure 44. Experiment 2 performance profile for multiplied probability methods on equality-free MIPs - computation time 


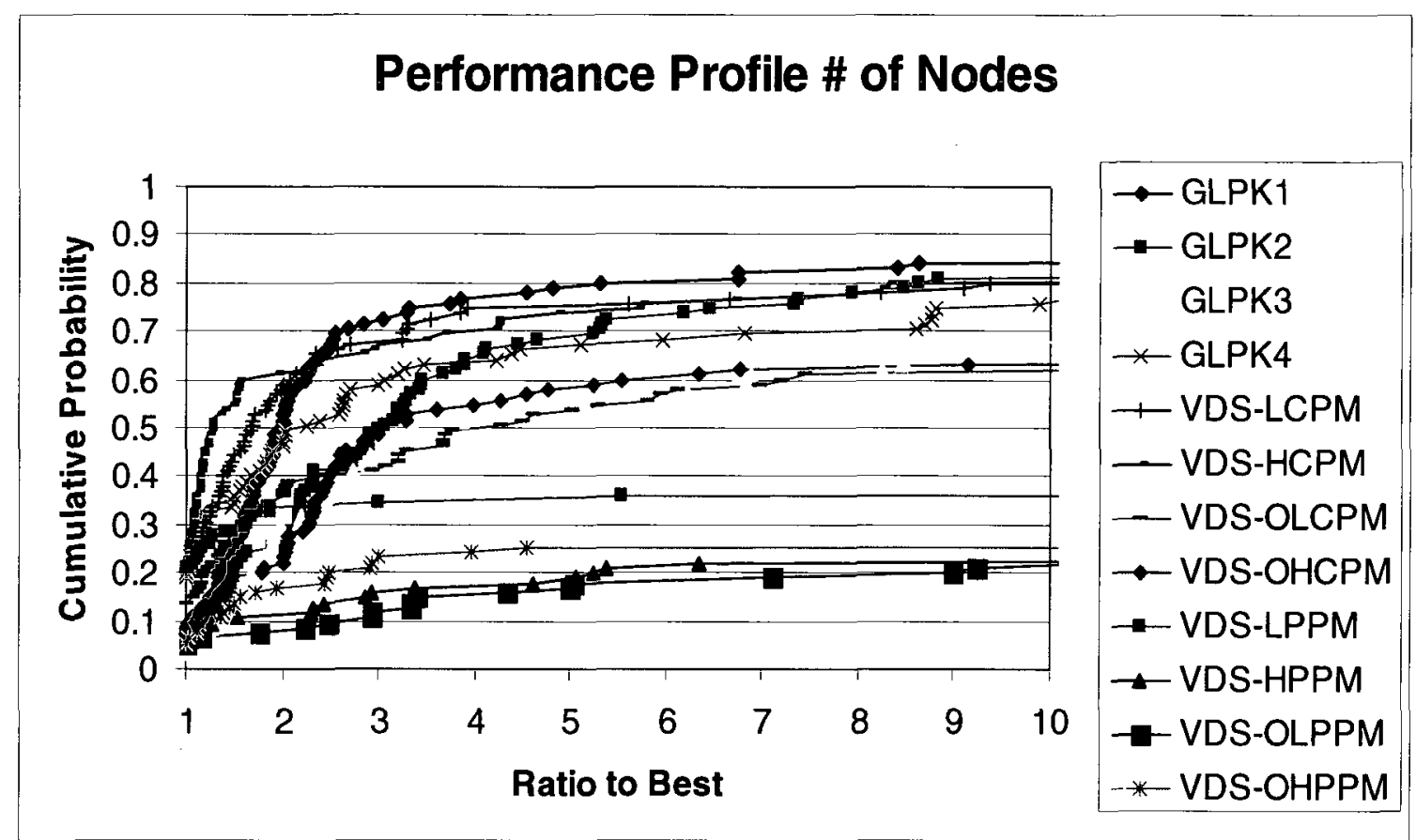

Figure 45. Experiment 2 performance profile for multiplied probability methods on equality-free MIPs - number of nodes

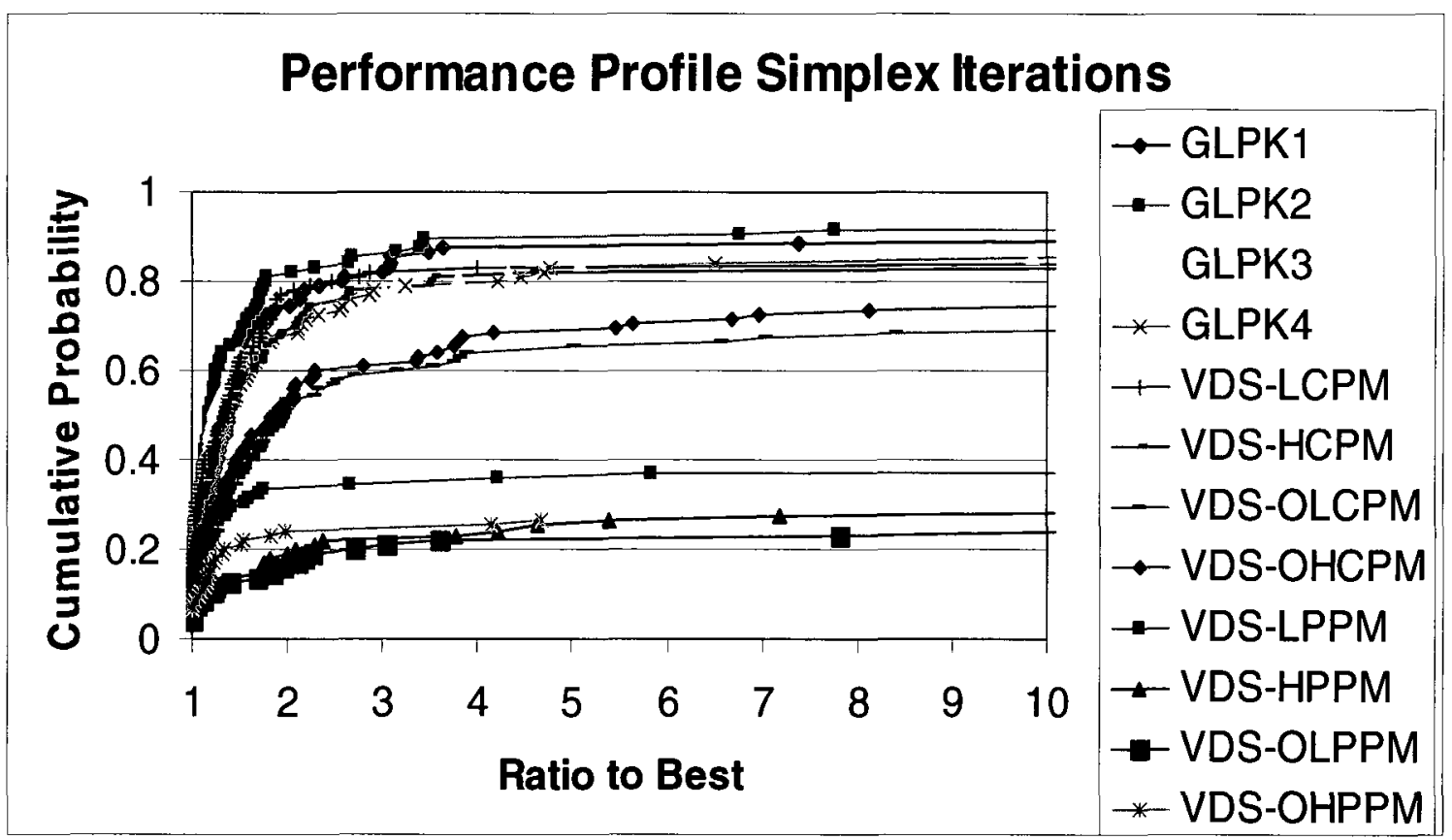

Figure 46. Experiment 2 performance profile for multiplied probability methods on equality-free MIPs - simplex iterations 


\subsection{Equality-Free versus Equality-Containing MIPs}

\subsubsection{Goal of Experiment}

The top probability method, VDS-LCP, is compared to all six variable/node selection combinations (see Table 7) over all of the MIPs (regardless of equality constraints) to see if VDS-LCP is competitive with the state of the art variable and direction selection algorithms. Then the data is separated by equality-free and equality-containing MIPs to examine the differences.

\subsubsection{Experimental Results}

Figure 47 below, displays the overall results for Experiment 2 in terms of time; it contains all of the ratios to best for each branching variable selection method over the entire MIP set, regardless of equalities in the MIP. Figure 48 and Figure 49 show the overall results for number of nodes, and simplex iterations respectively.

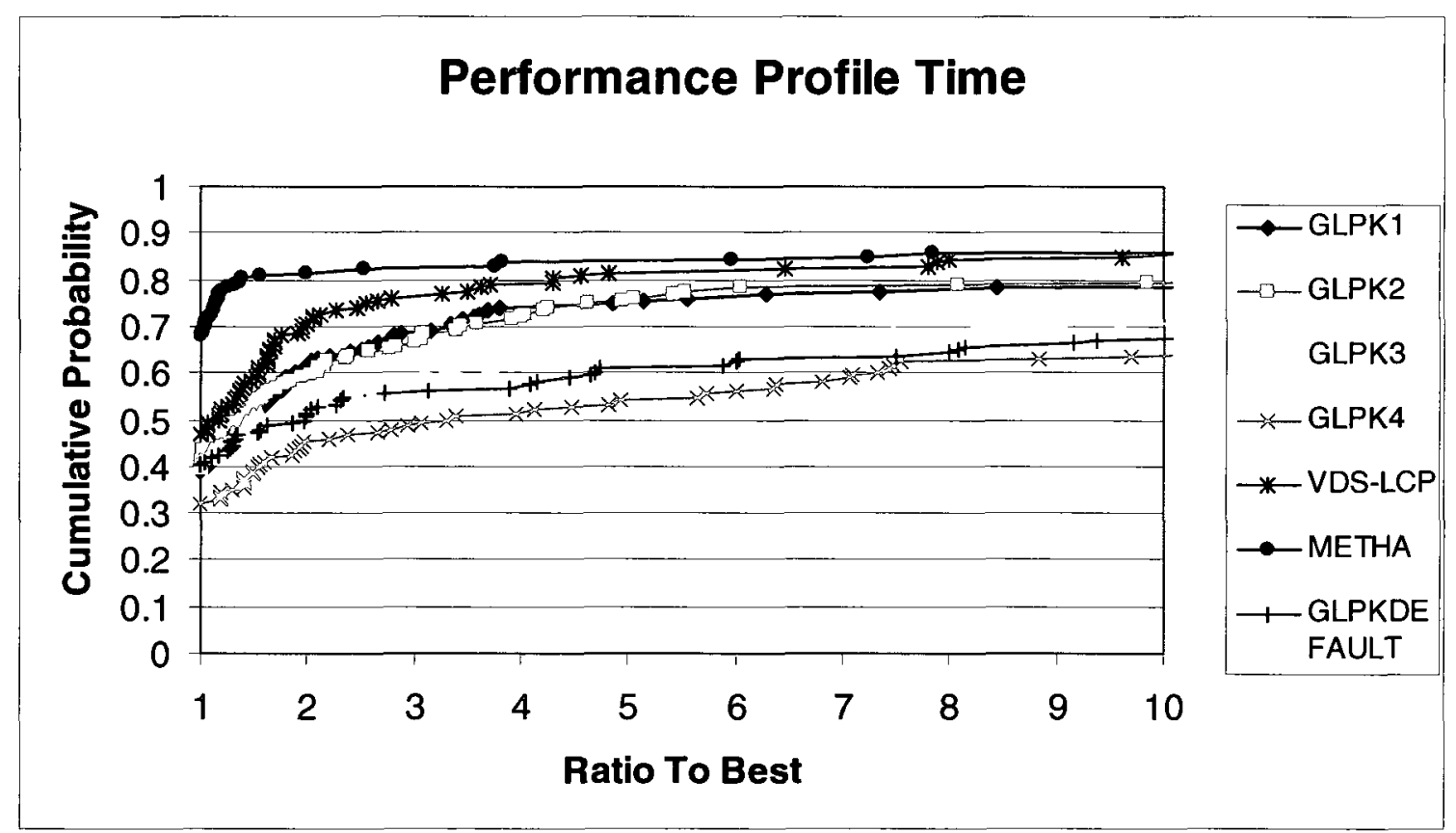

Figure 47. Experiment 2 performance profile for overall data - computation time 


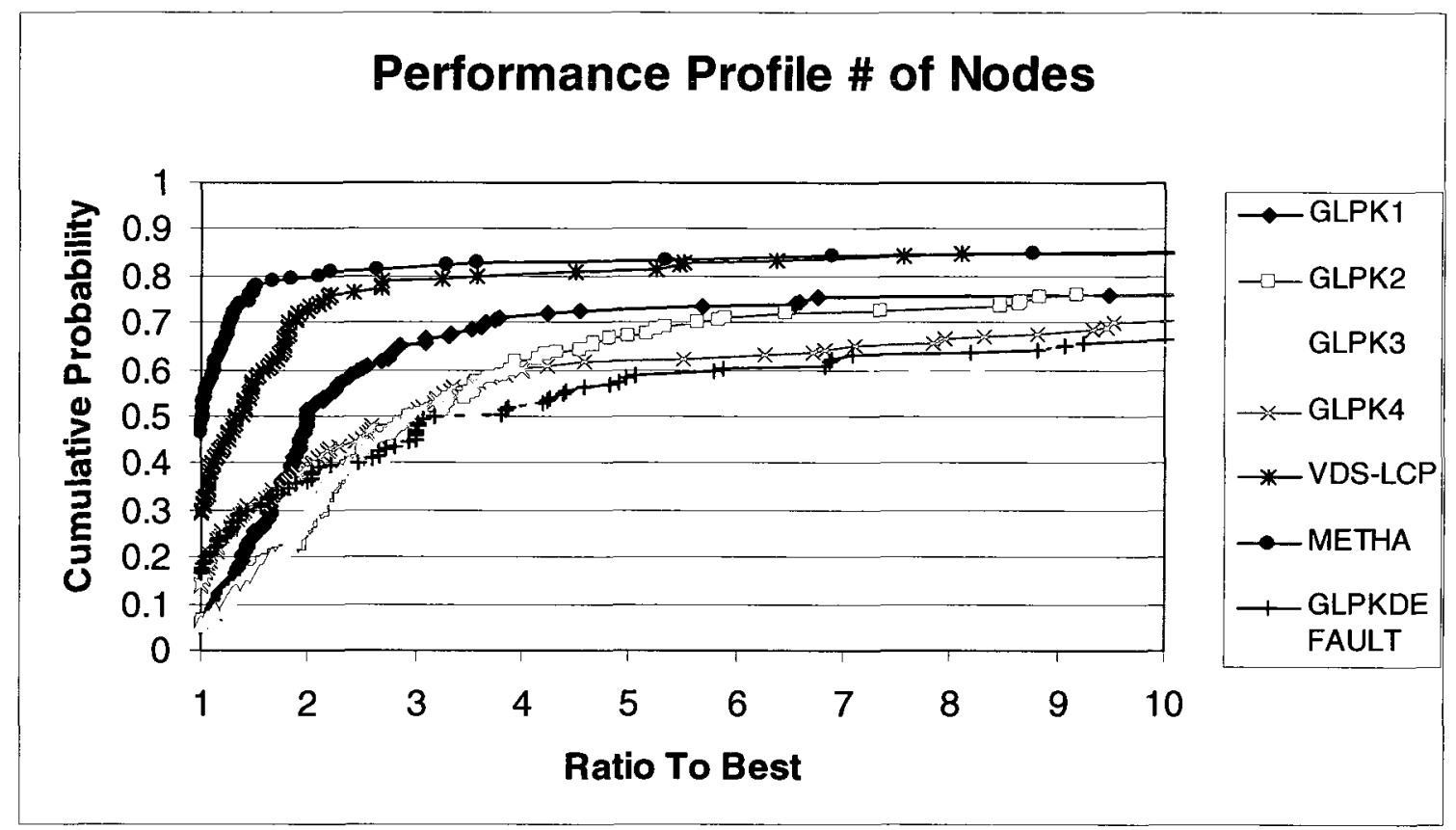

Figure 48. Experiment 2 performance profile for overall data - number of nodes

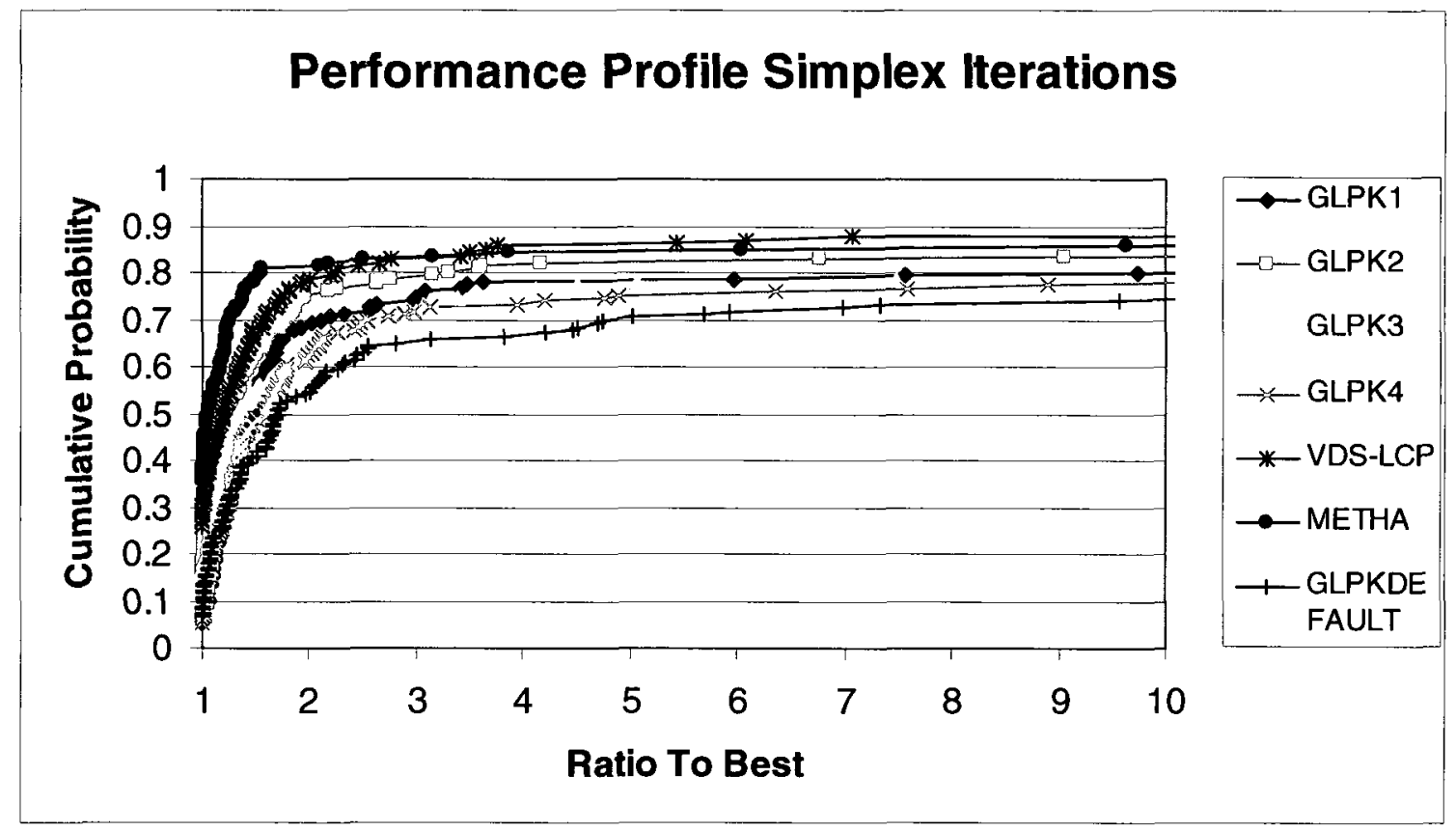

Figure 49. Experiment 2 performance profile for overall data - simplex iterations

From these graphs, METHA, the active-constraints method, is the overall winner. However, VDS-LCP is a close competitor to METHA, leaving a gap between the two methods and all five of the GLPK branching variable selection methods. VDS-LCP 
provides an improvement over the GLPK methods on a general set of MIPs on all performance metrics.

VDS-LCP is more robust than METHA in terms of the number of MIPs completed within the two hour time limit. The number of dives required to reach feasibility is also analyzed for VDS-LCP and METHA over the entire MIP set. On 115 of the 133 MIPs evaluated (where both methods do not time out, see list in Appendix A.5), VDS-LCP and METHA both only require one dive to reach feasibility. For the remaining 18 MIPs, VDS-LCP performs fewer dives than METHA on 12 of the MIPs. As mentioned earlier, the number of dives to reach first feasibility is another measure of the quality of a B\&B algorithm. VDS-LCP is better than METHA in terms of number of dives to reach first feasibility.

In addition to looking at the overall set of MIPs, the dataset was split into equality-containing and equality-free subsets. This proved to be a useful separation, as METHA's domination of VDS-LCP is clear in the equality-free MIPs, however, VDSLCP slightly outperforms METHA when comparing the equality-containing MIPs. VDSLCP is also more robust than METHA on this subset of MIPs. The equality-free MIP set results are shown in Figure 50, Figure 51, and Figure 52 and the equality-containing MIP set results are shown in Figure 53, Figure 54, and Figure 55. 


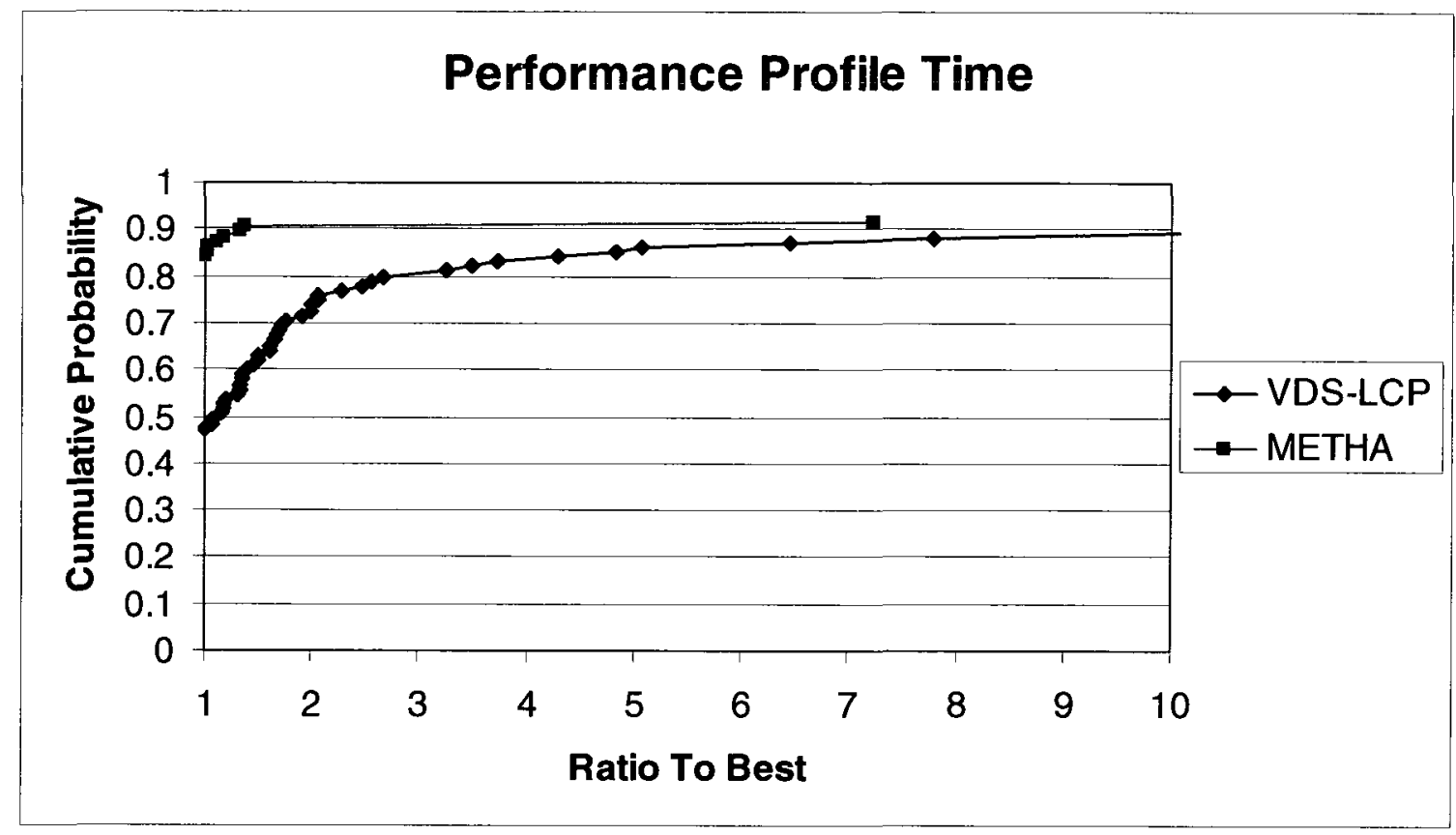

Figure 50. Experiment 2 performance profile for equality-free MIPs - computation time

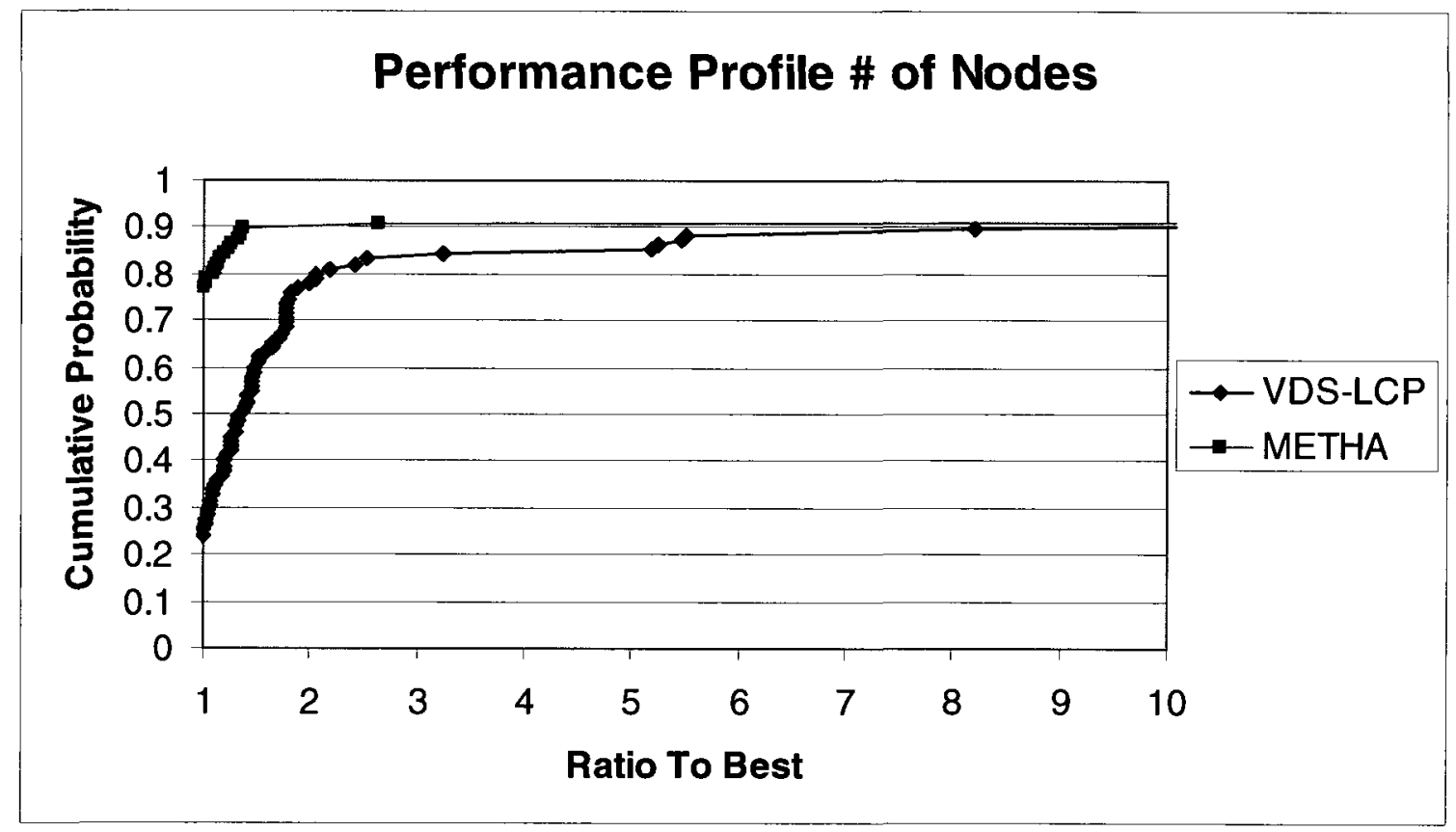

Figure 51. Experiment 2 performance profile for equality-free MIPs - number of nodes 


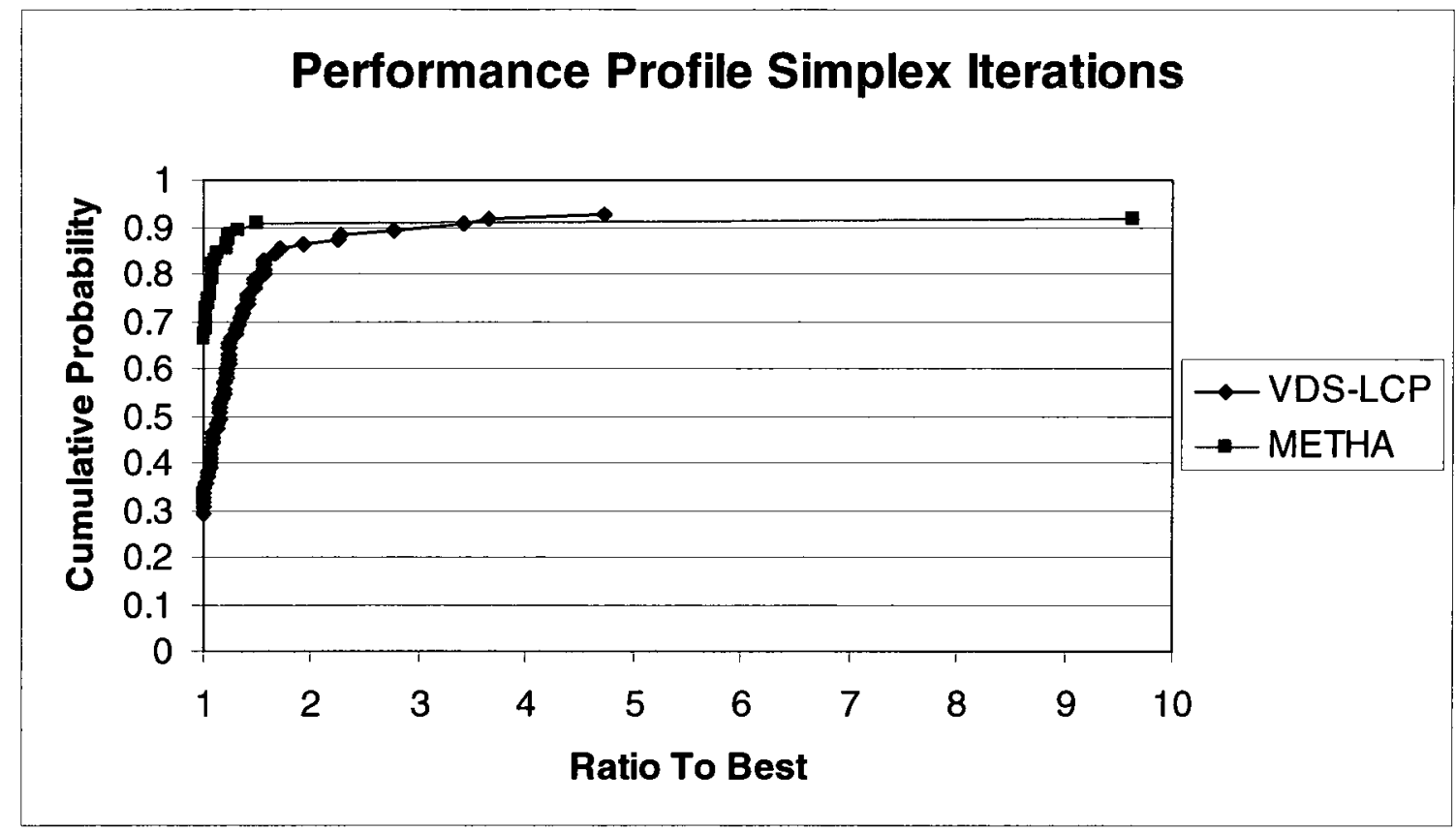

Figure 52. Experiment 2 performance profile for equality-free MIPs - simplex iterations

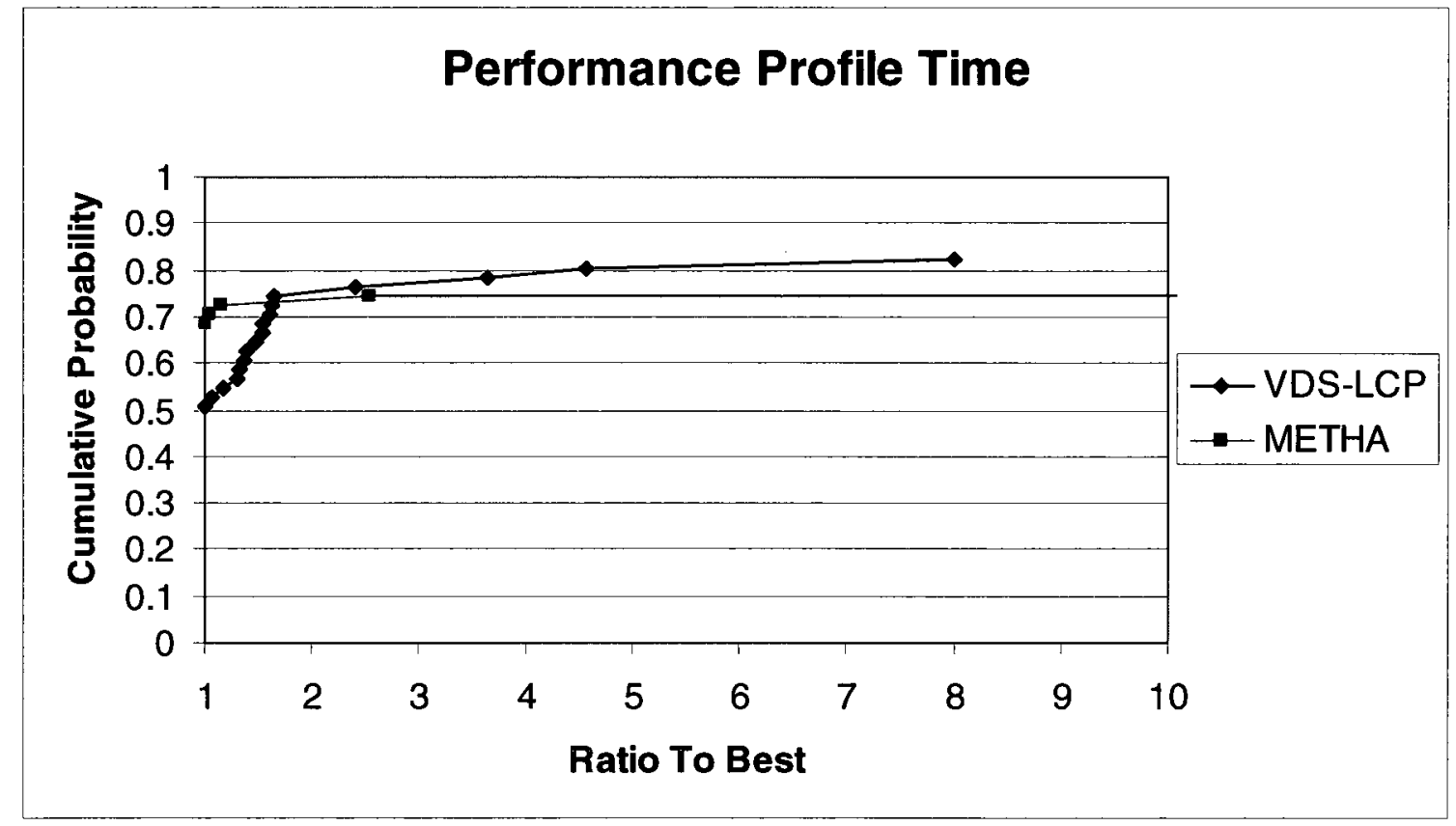

Figure 53. Experiment 2 performance profile for equality-containing MIPs - computation time 


\section{Performance Profile \# of Nodes}

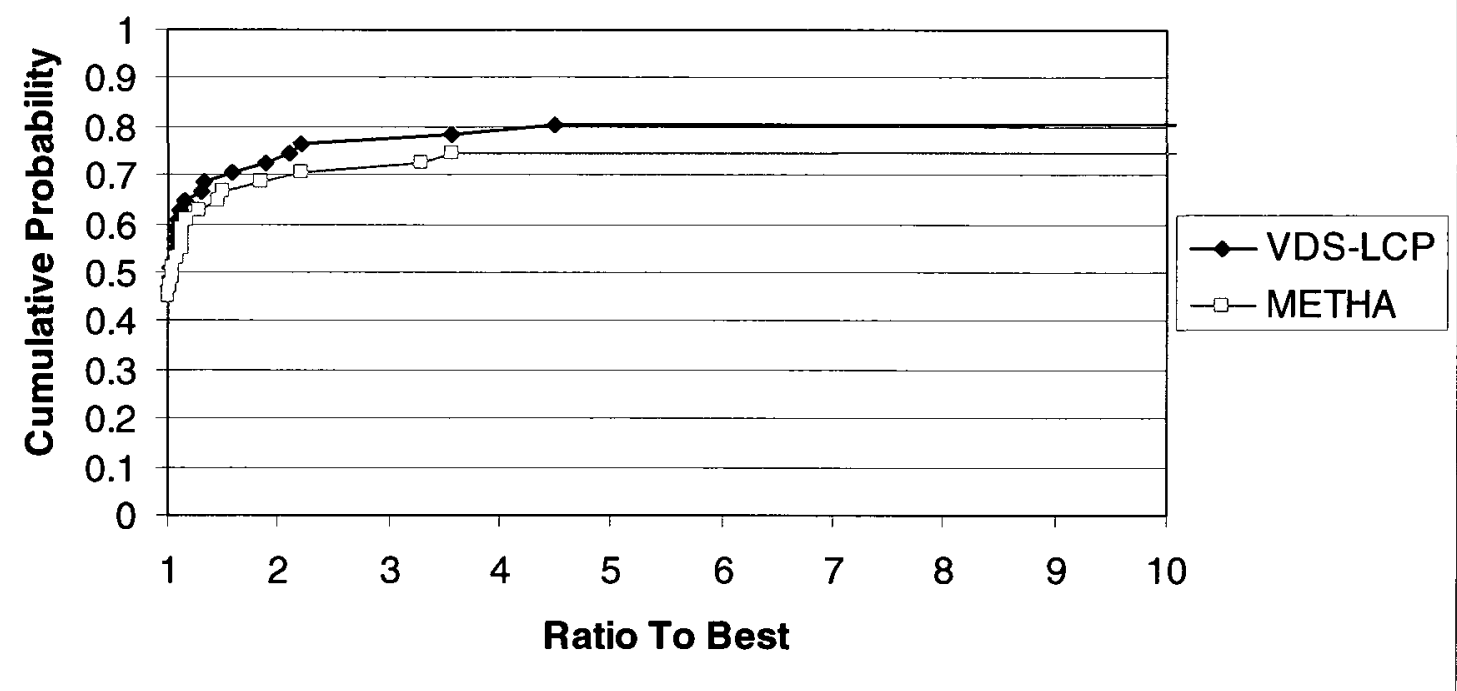

Figure 54. Experiment 2 performance profile for equality-containing MIPs - number of nodes

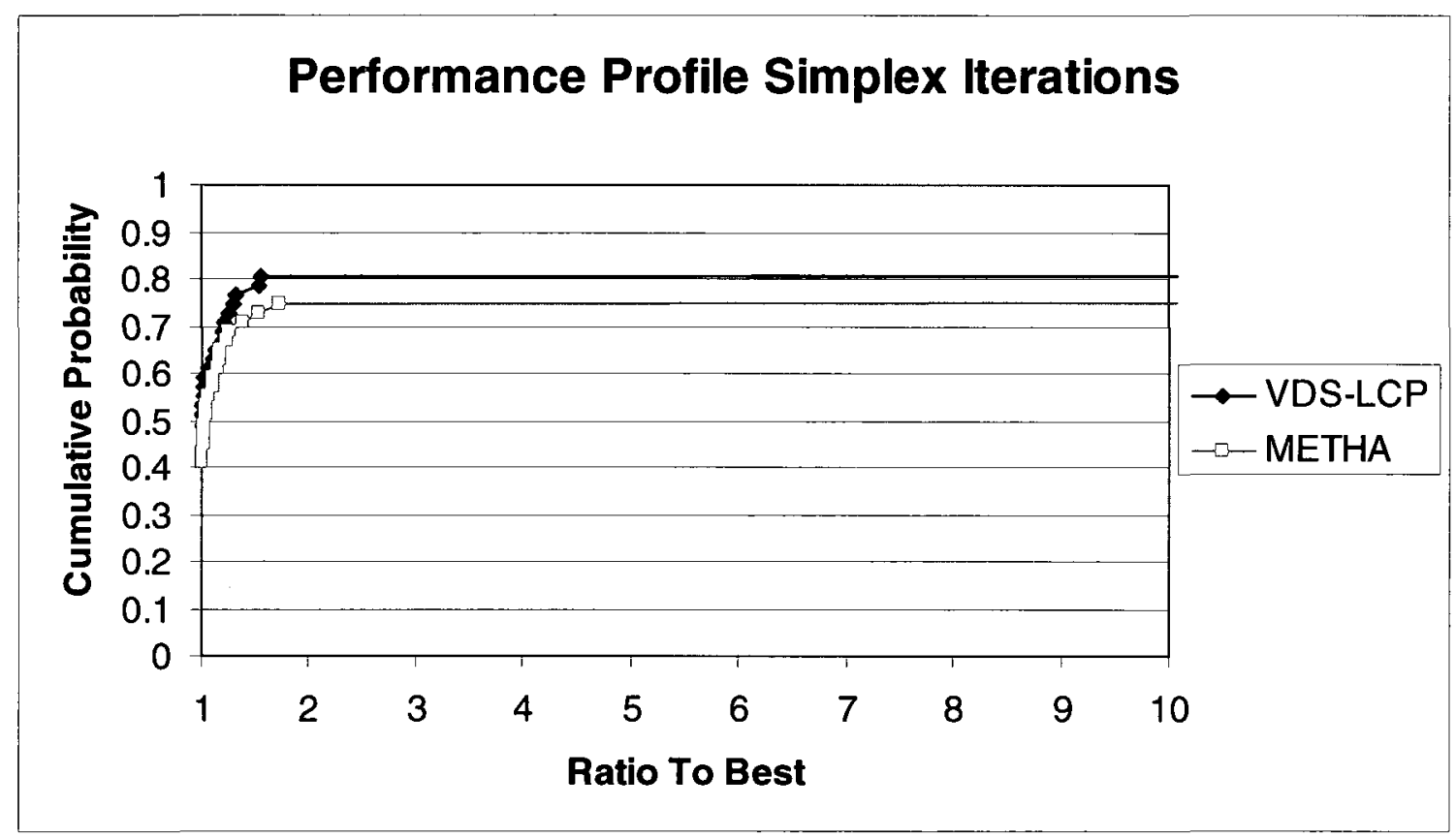

Figure 55. Experiment 2 performance profile for equality-containing MIPs - simplex iterations 


\subsection{Hybrid Methods}

\subsubsection{Goal of Experiment}

Four hybrid methods (see Table 6) are compared against the top variable and direction selection method, METHA, to explore the patterns noticed in earlier experiments. One hybrid method in Table 6, HYBRID2, is not discussed in this section. It is addressed in Section 9.3. All of the hybrid methods are tested on the entire MIP set.

\subsubsection{Experimental Results}

As mentioned in Section 5.3.4, HYBRID1 uses VDS-LCP if it is solving an equalitycontaining MIP and METHA if it is solving an equality-free MIP. This method performs slightly, but not significantly, better than METHA by itself. It is also more robust than METHA alone. The performance profiles comparing METHA and HYBRID1 are shown below in Figure 56, Figure 57, and Figure 58.

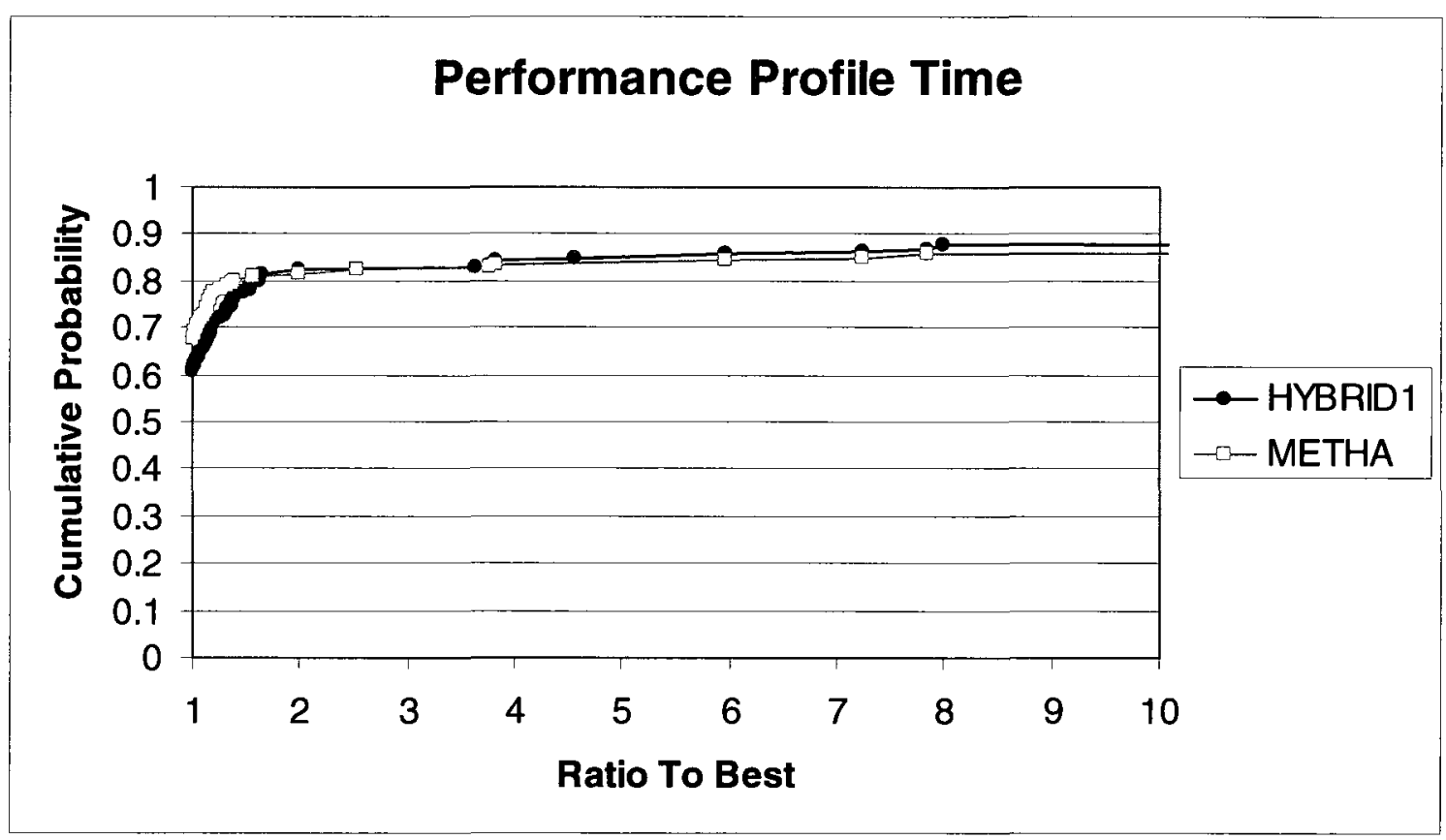

Figure 56. Experiment 2 performance profile for HYBRID1 - computation time 


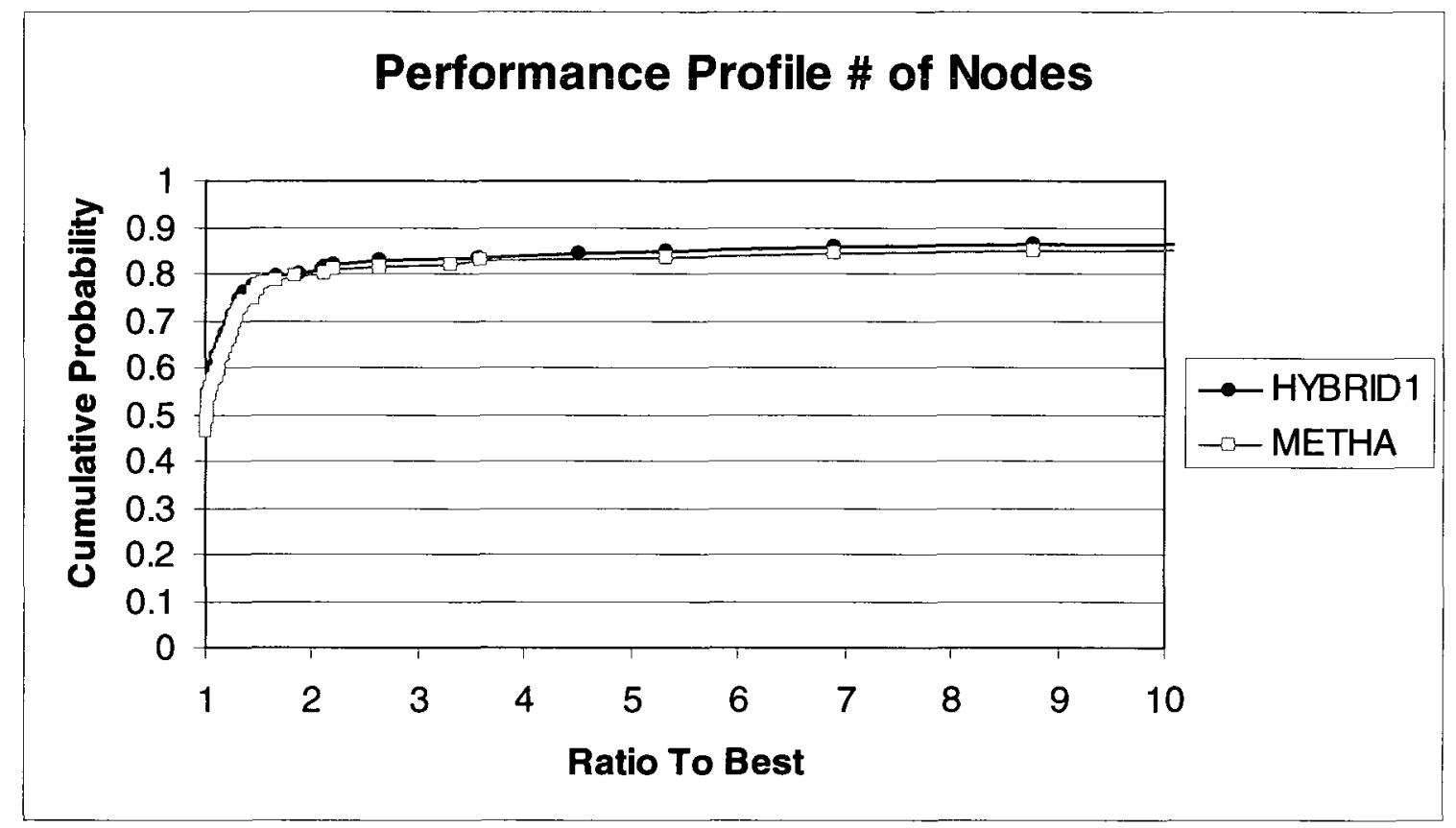

Figure 57. Experiment 2 performance profile for HYBRID1 - number of nodes

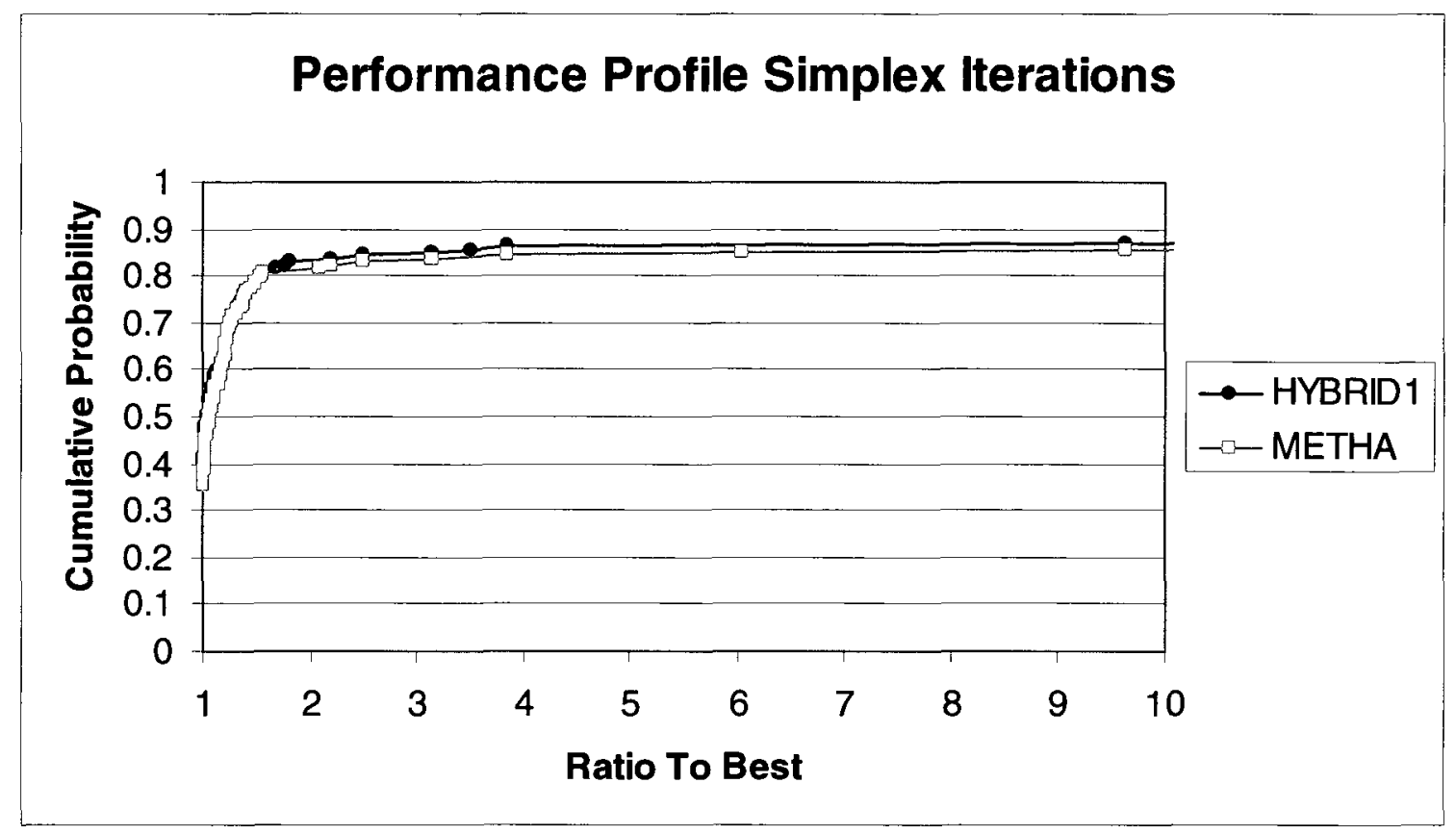

Figure 58. Experiment 2 performance profile for HYBRID1 - simplex iterations

TIES (common ties subset) is slower than just performing METHA because of the extra computation required: at each node, the algorithm generates the branching variable ties list for both VDS-LCP and METHA, and then compares the lists for the first 
common variable. This is reflected on the computation time performance profile in Figure 59 below. However, in terms of number of nodes and simplex iterations, TIES performs slightly better than METHA. This is seen in Figure 60, and Figure 61 below. TIES also does slightly better than METHA when just looking at the subset of equalityfree MIPs (except for in terms of time). In both cases, METHA is more robust than TIES.

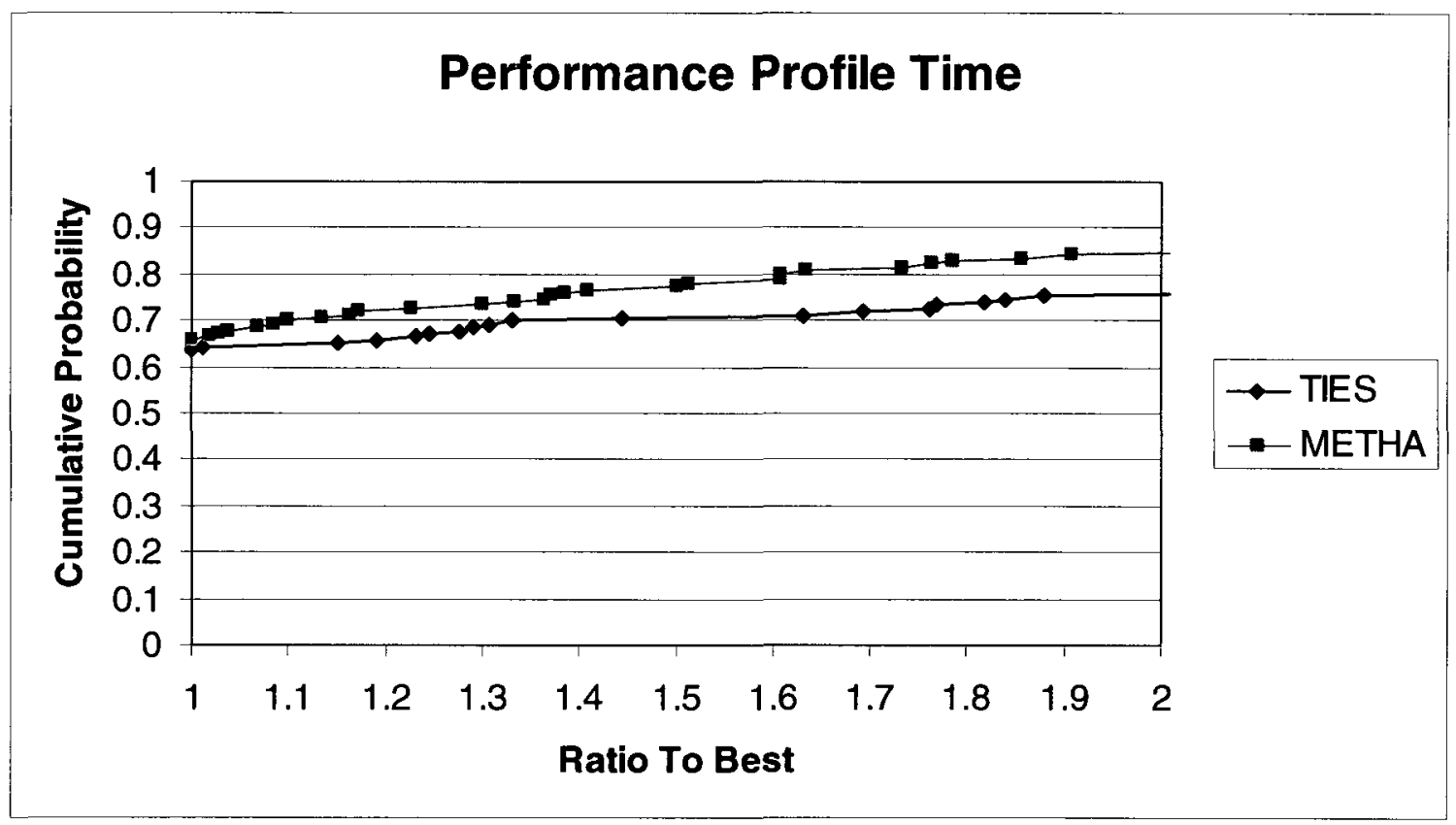

Figure 59. Experiment 2 performance profile for TIES - computation time 


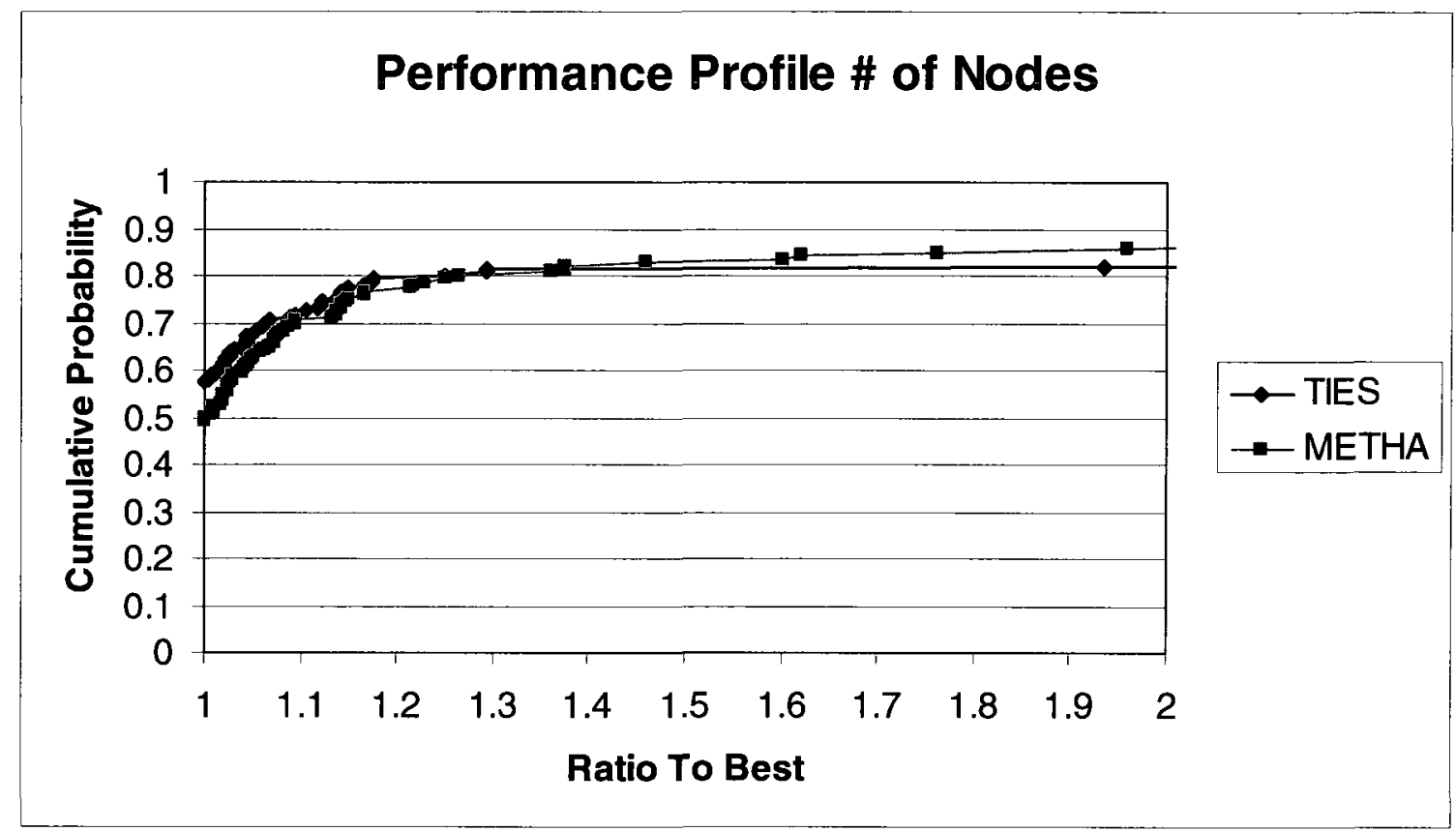

Figure 60. Experiment 2 performance profile for TIES - number of nodes

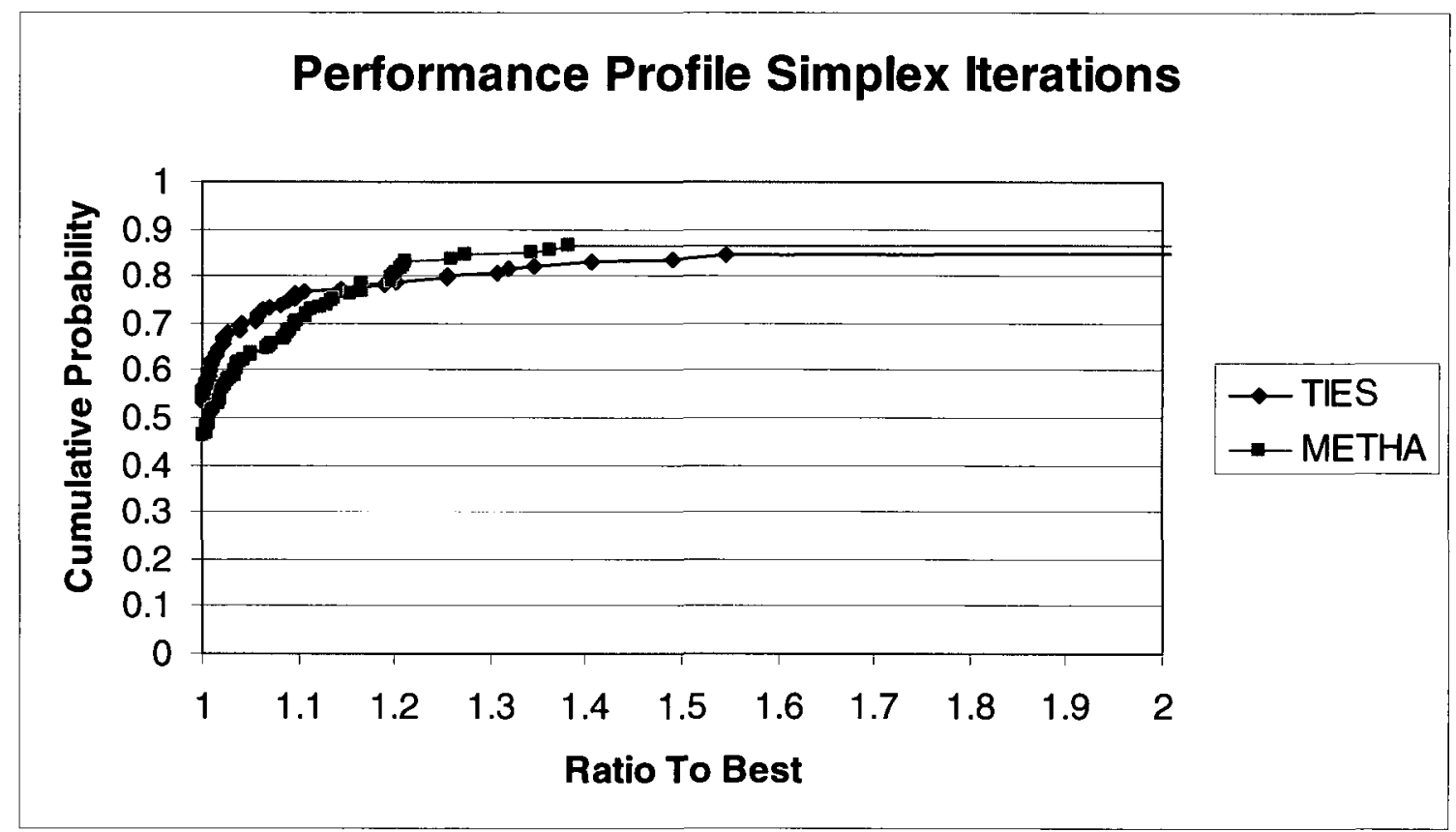

Figure 61. Experiment 2 performance profile for TIES - simplex iterations

In Experiment 1, branching up is the best branching variable direction selection heuristic. This provided the incentive to try VDS-LCP-UP1. When compared against 
METHA on the entire MIP set, VDS-LCP-UP1 has the same robustness as METHA, and both methods perform nearly identically on the three performance profiles shown below.

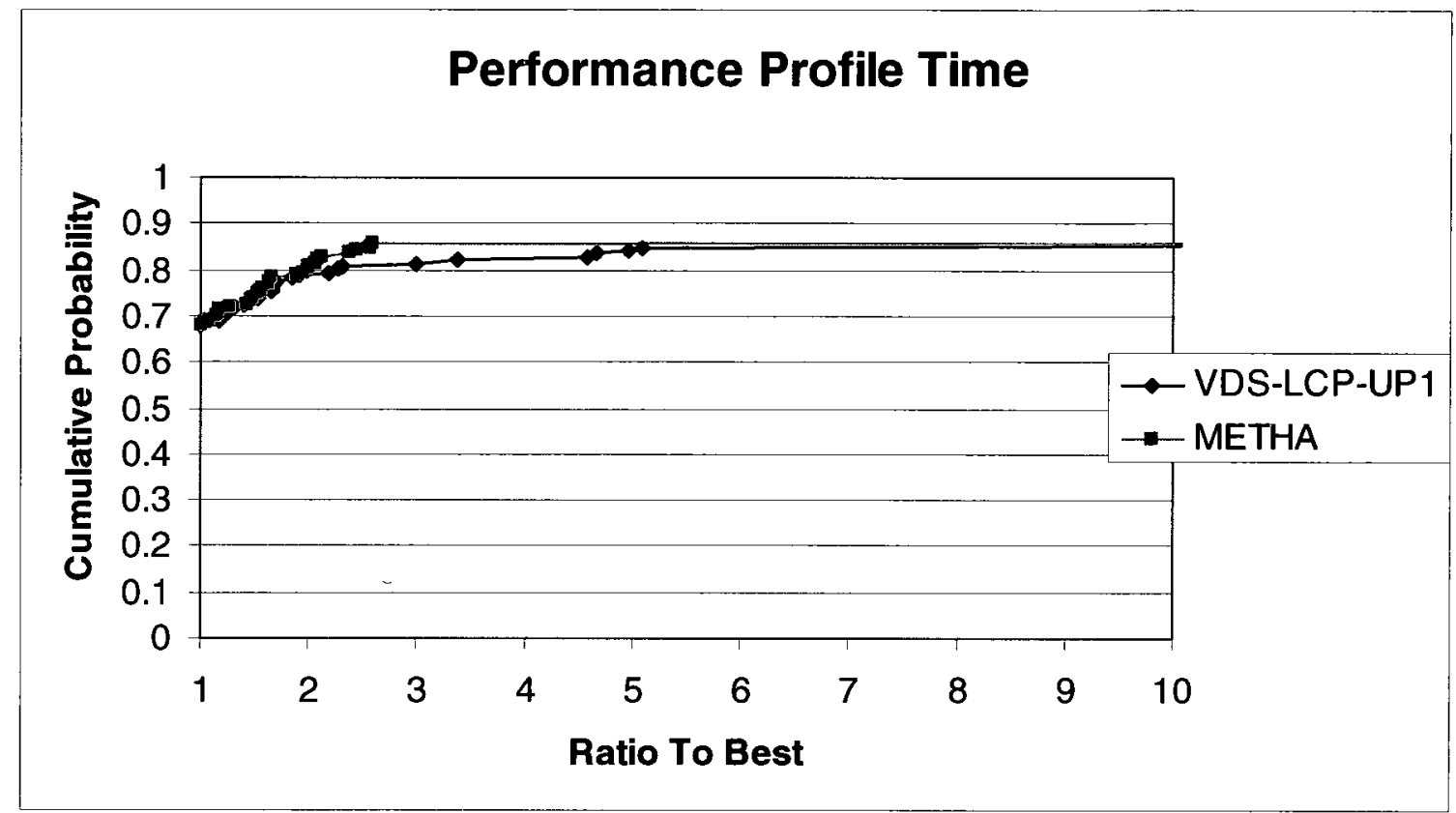

Figure 62. Experiment 2 performance profile for VDS-LCP-UP1 - computation time

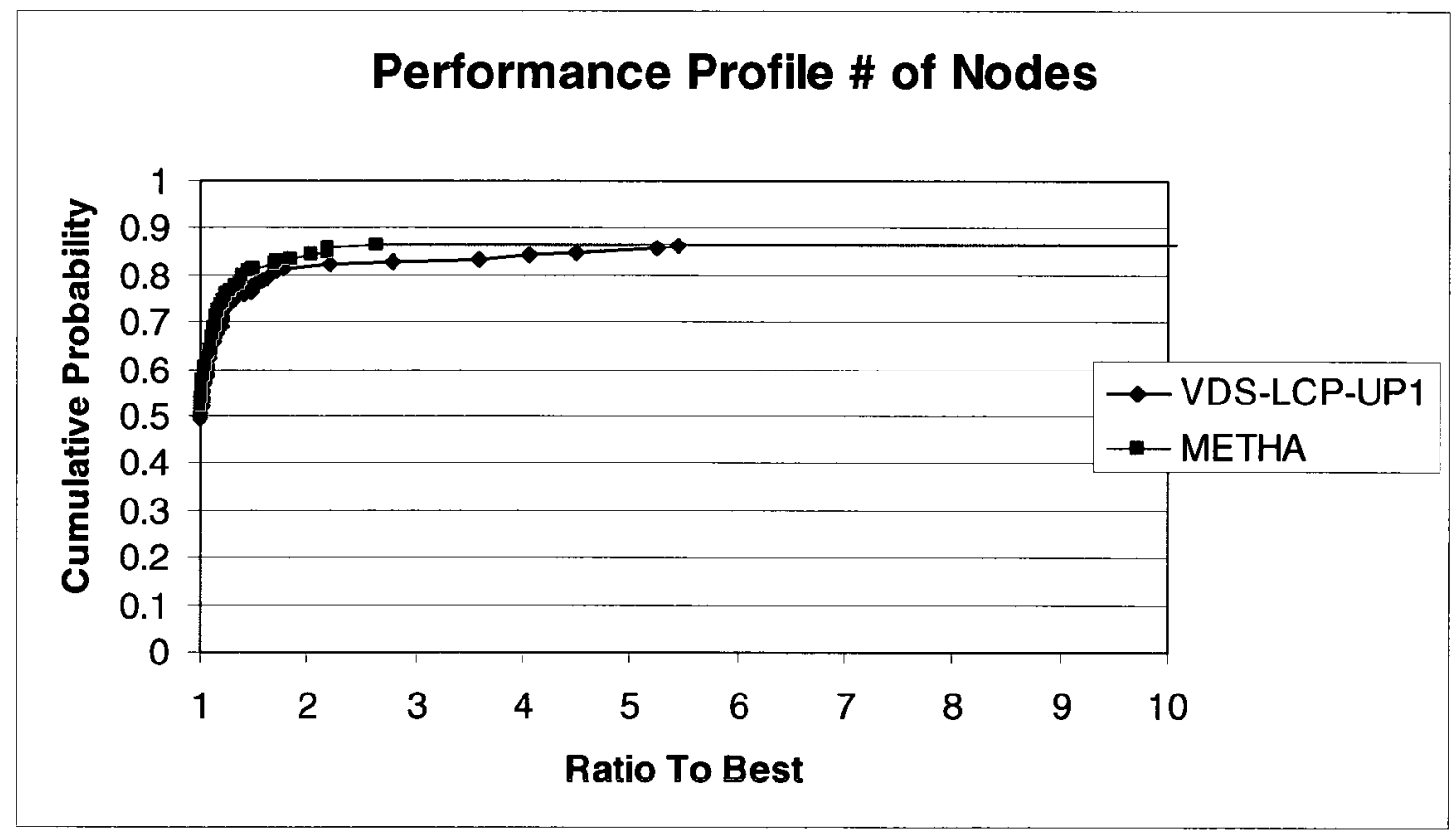

Figure 63. Experiment 2 performance profile for VDS-LCP-UP1 - number of nodes 


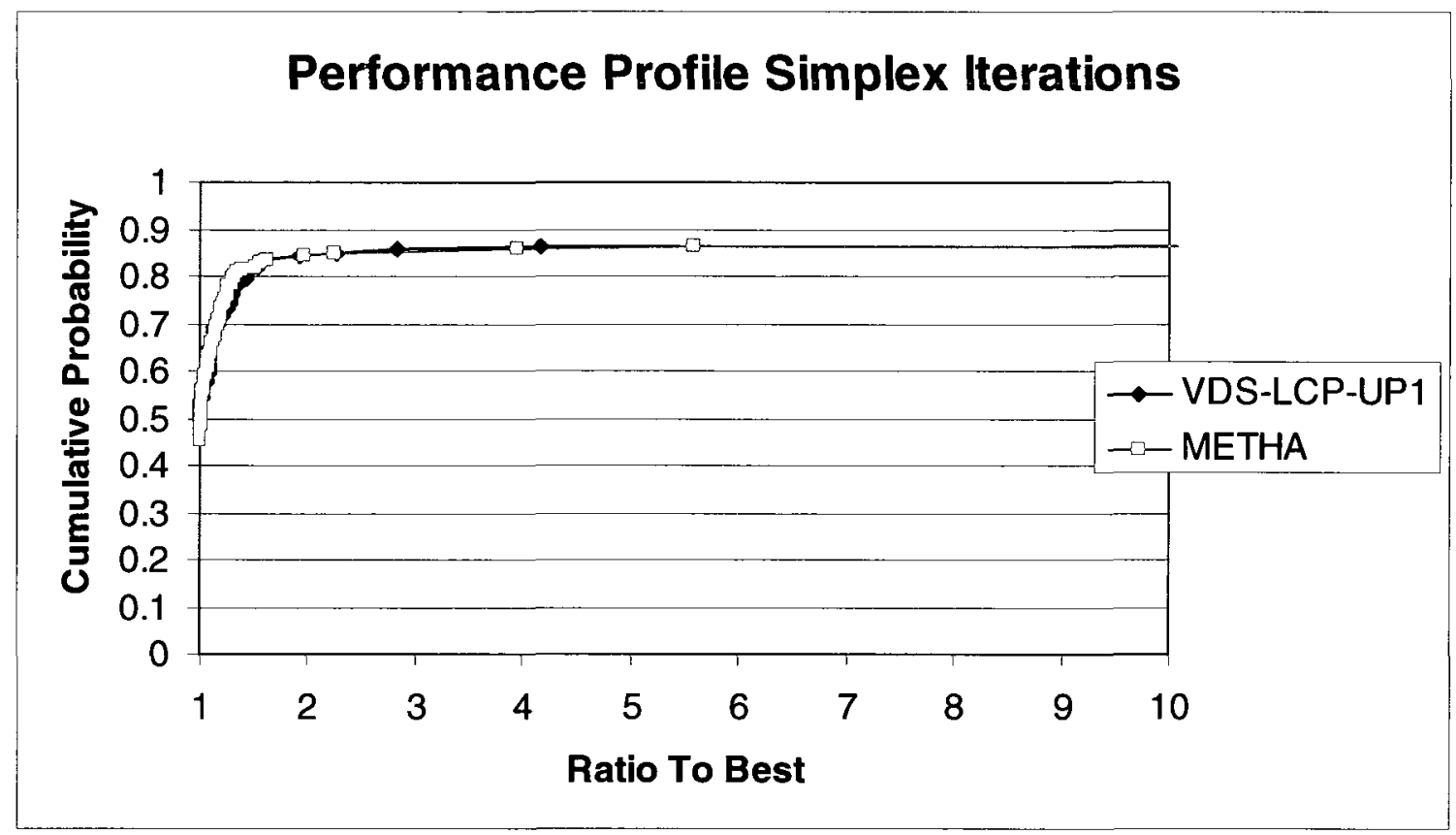

Figure 64. Experiment 2 performance profile for VDS-LCP-UP1 - simplex iterations

The empirical results for VDS-LCP-UP2 are shown in the three performance profiles below. This method was tested due to the fact that VDS-LCP-UP1 performs well on the equality-free MIPs. As mentioned in Section 5.3.4 this hybrid uses VDS-LCP-UP1 when solving an equality-free MIP, otherwise VDS-LCP is used. It is compared to METHA, and it results in a more robust competitor for METHA. The hybrid even does slightly better than METHA in terms of simplex iterations, while being nearly identical in terms of time and number of nodes. 


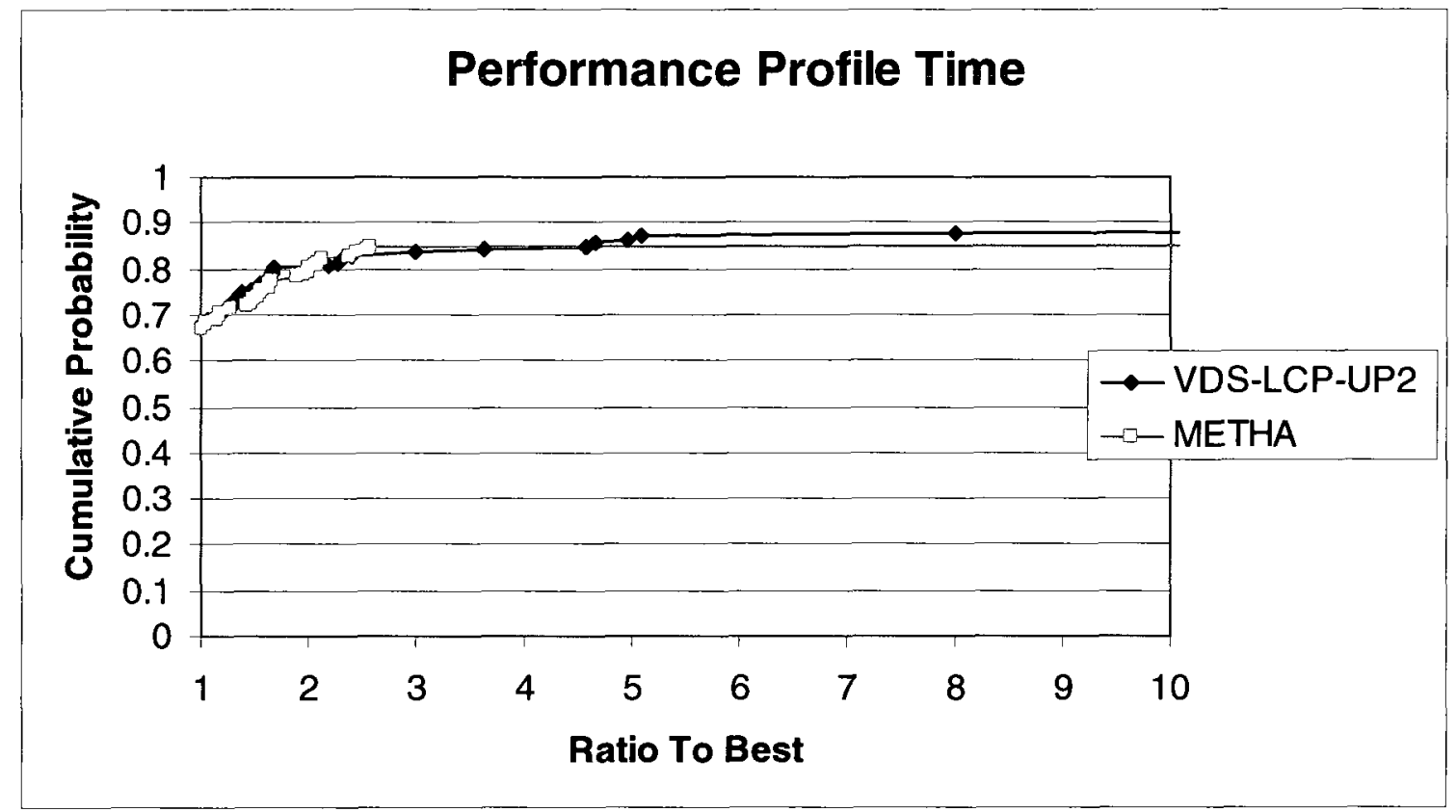

Figure 65. Experiment 2 performance profile for VDS-LCP-UP2 - computation time

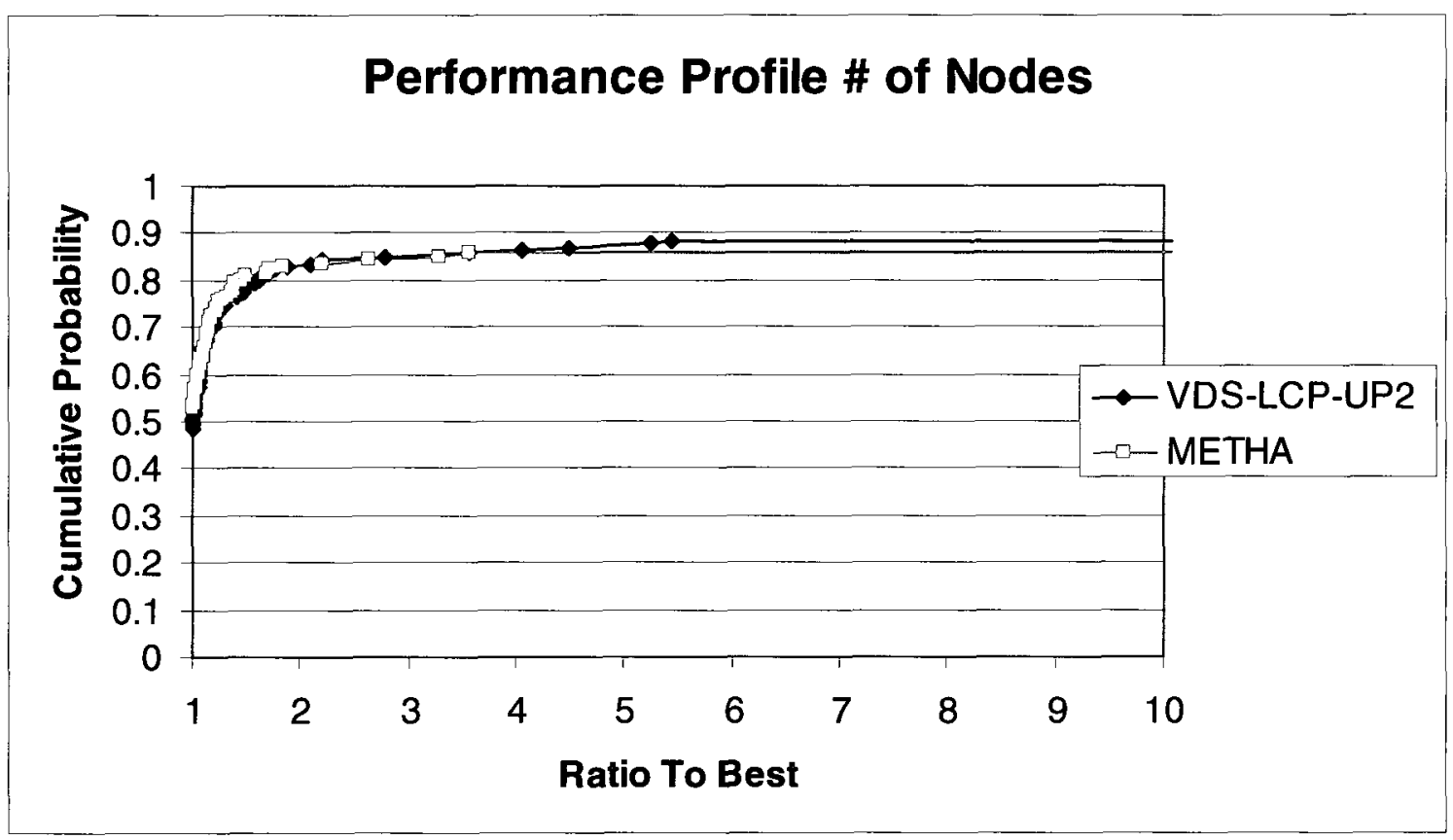

Figure 66. Experiment 2 performance profile for VDS-LCP-UP2 - number of nodes 


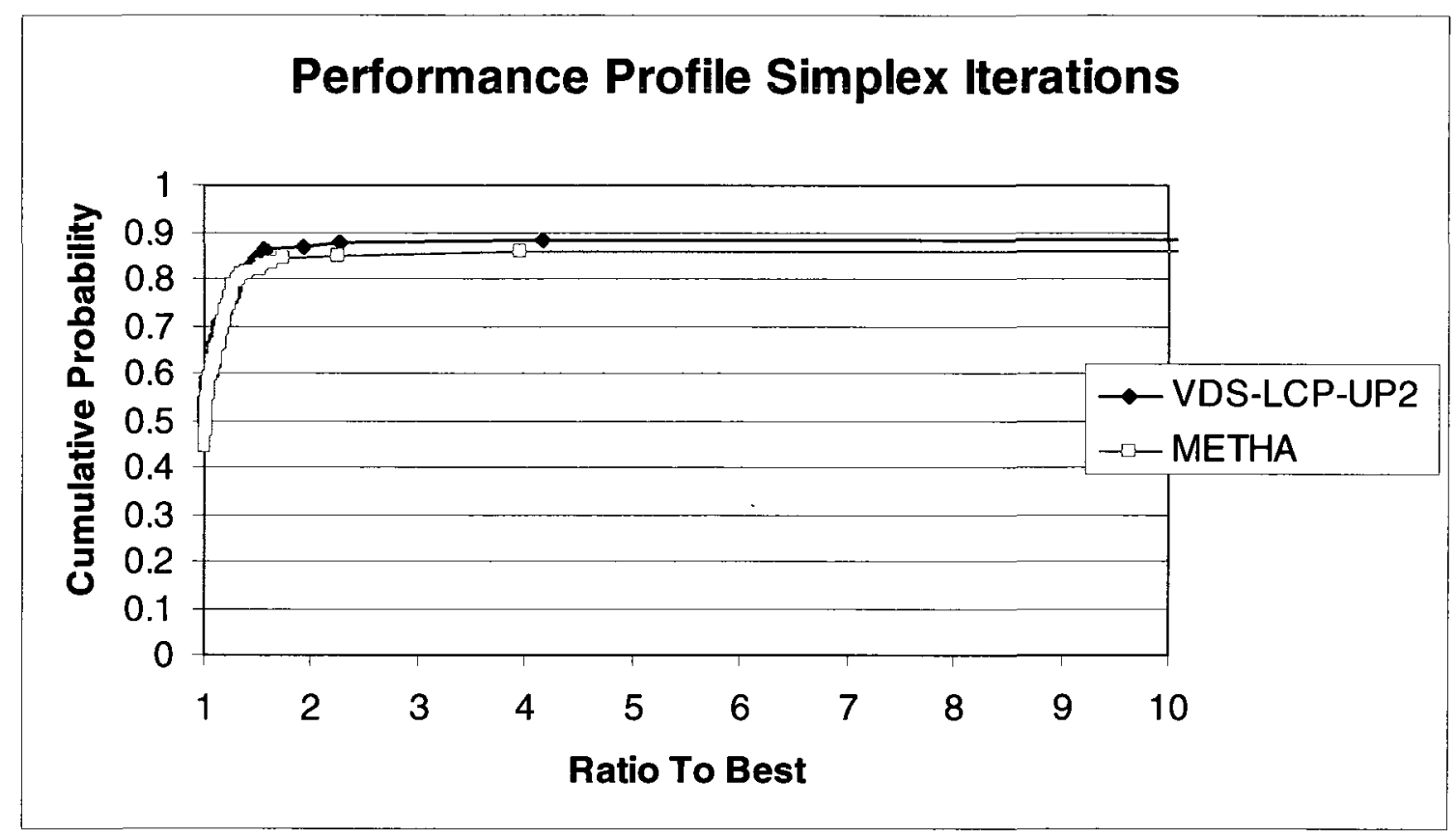

Figure 67. Experiment 2 performance profile for VDS-LCP-UP2 - simplex iterations

Overall, the main result from Experiment 2 is that VDS-LCP out-performs the GLPK methods on a general set of MIPs. In addition, VDS-LCP is competitive with the state of the art in reaching integer feasibility quickly when evaluating equality-containing MIPs. 


\section{Discussion of Results}

Branching up is, in general, the best branching direction heuristic. This section seeks an explanation for this phenomenon. It will also shed light on why the most violated votes method is the second best method. Finally, it will discuss why the lowest cumulative probability method works well on MIPs with equality constraints. In general, the unifying theme is that branching in the direction that violates a constraint forces multiple variables to adjust because their freedom of choice is reduced.

\subsection{Role of Multiple Choice Constraints}

A multiple choice constraint is an equality or less-than inequality constraint which contains only binary variables and whose RHS value is one. All of the variable coefficients in a multiple choice constraint are one. A multiple choice constraint takes the form of

$$
\begin{gathered}
\sum_{i=1}^{n} x_{i}=1 \\
\text { or } \\
\sum_{i=1}^{n} x_{i} \leq 1,
\end{gathered}
$$

where all of the $n$ variables are binary.

When there are one or more candidate variables in a multiple choice constraint, selecting one as the branching variable and branching in the up direction (forcing its value to one), automatically forces the remaining candidate variables to zero. This action 
forces all of the variables in the multiple choice constraint to integrality simultaneously. Reducing the candidate variable list quickly allows for the speedier achievement of reaching an integer feasible MIP solution.

On the other hand, if we branch down on a candidate variable in a multiple choice constraint, this does not force the remaining variables to integer values. This logic implies that branching up on a candidate variable in a multiple choice constraint is the better choice because it simultaneously forces other candidate variables to integrality.

Looking at the MIP set characteristics, the majority of the MIPs tested contain at least one multiple choice constraint. Only 38 of the $146 \mathrm{MIPs}$ tested do not contain any form of multiple choice constraint. This gives UP a distinct advantage over the other branching variable direction selection methods. When the results of Experiment 1 are split into two groups: MIPs with multiple choice constraints and MIPs without multiple choice constraints, this idea is confirmed. On the MIPs with multiple choice constraints, UP is the winning method; however, on the MIPs without multiple choice constraints three of the branching direction methods (UP, LCPV, and MVV) tie for best method. The latter three summary plots are noisier in addition to not having a clear best branching direction method. These results are shown in the following six figures. 


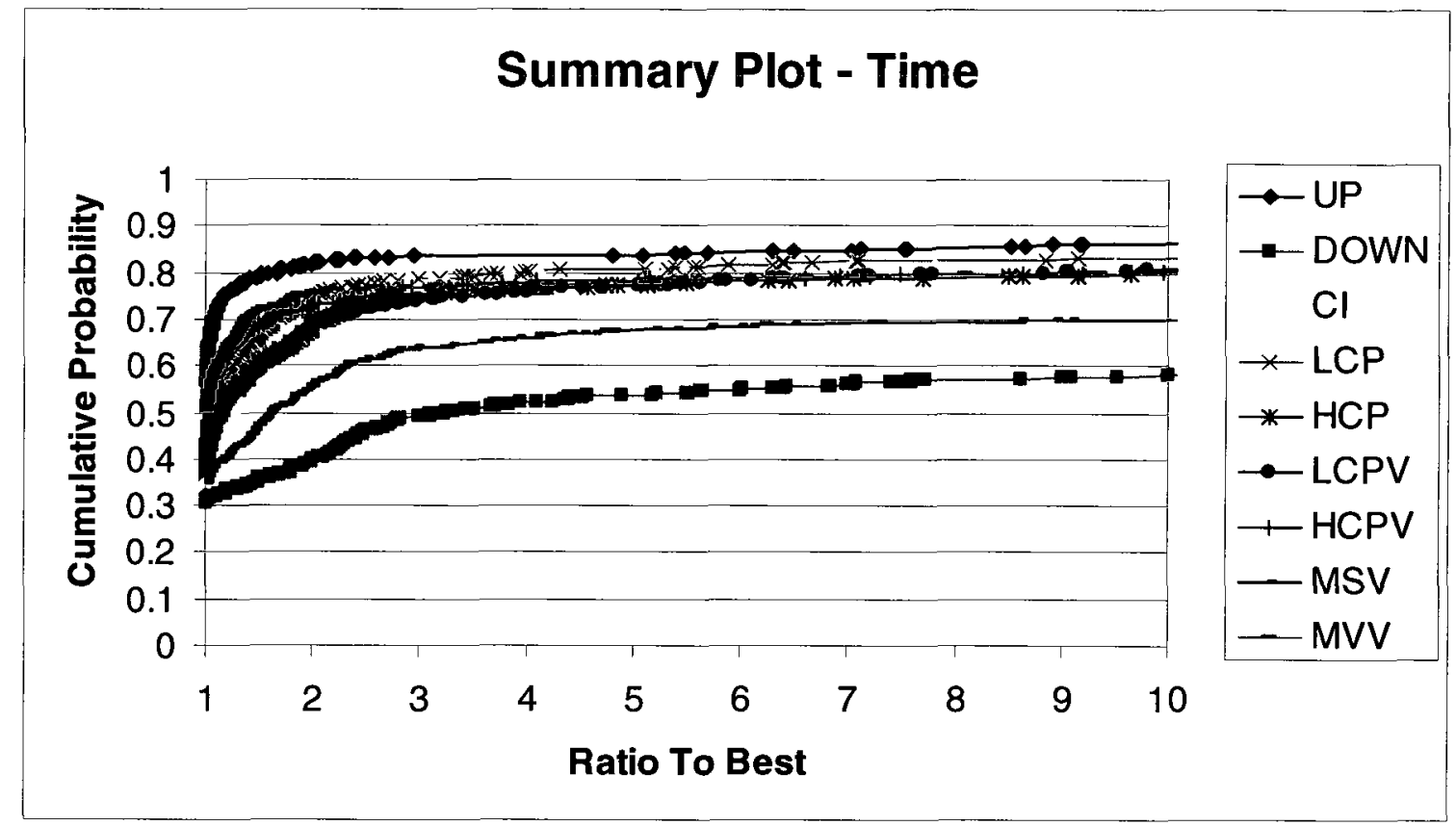

Figure 68. Experiment 1 summary plots for MIPs with multiple choice constraintscomputation time

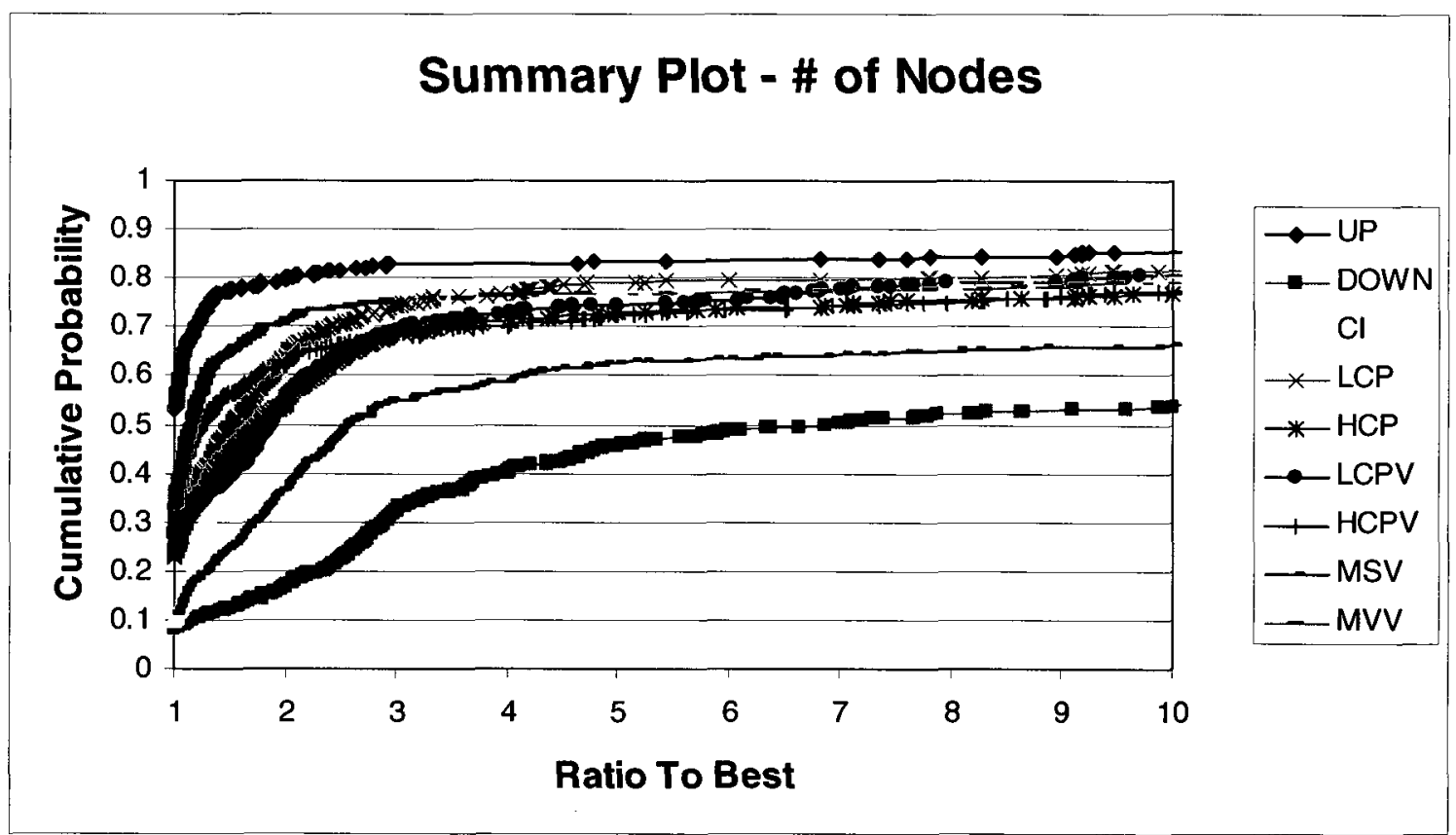

Figure 69. Experiment 1 summary plots for MIPs with multiple choice constraints number of nodes 


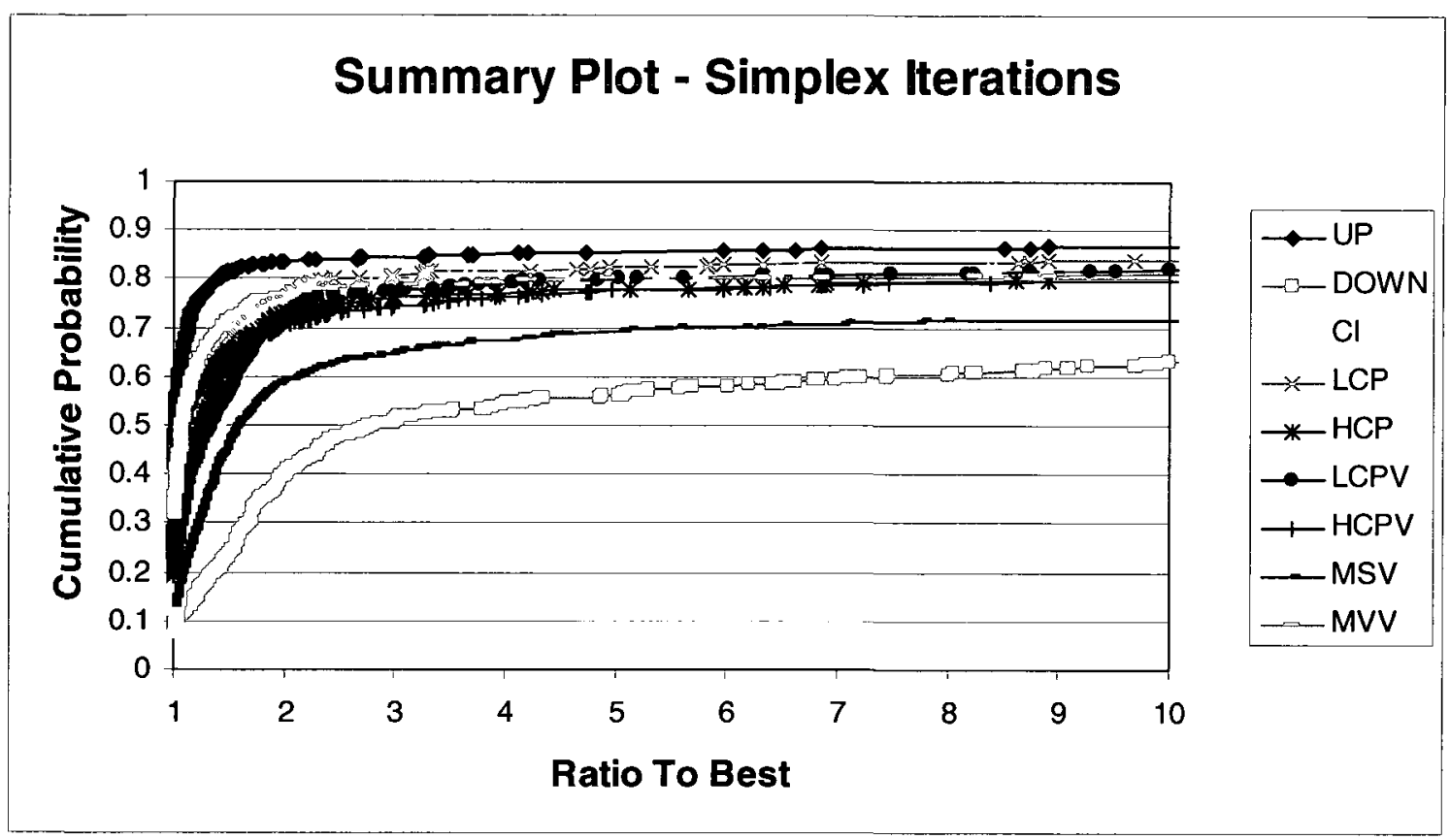

Figure 70. Experiment 1 summary plots for MIPs with multiple choice constraints simplex iterations

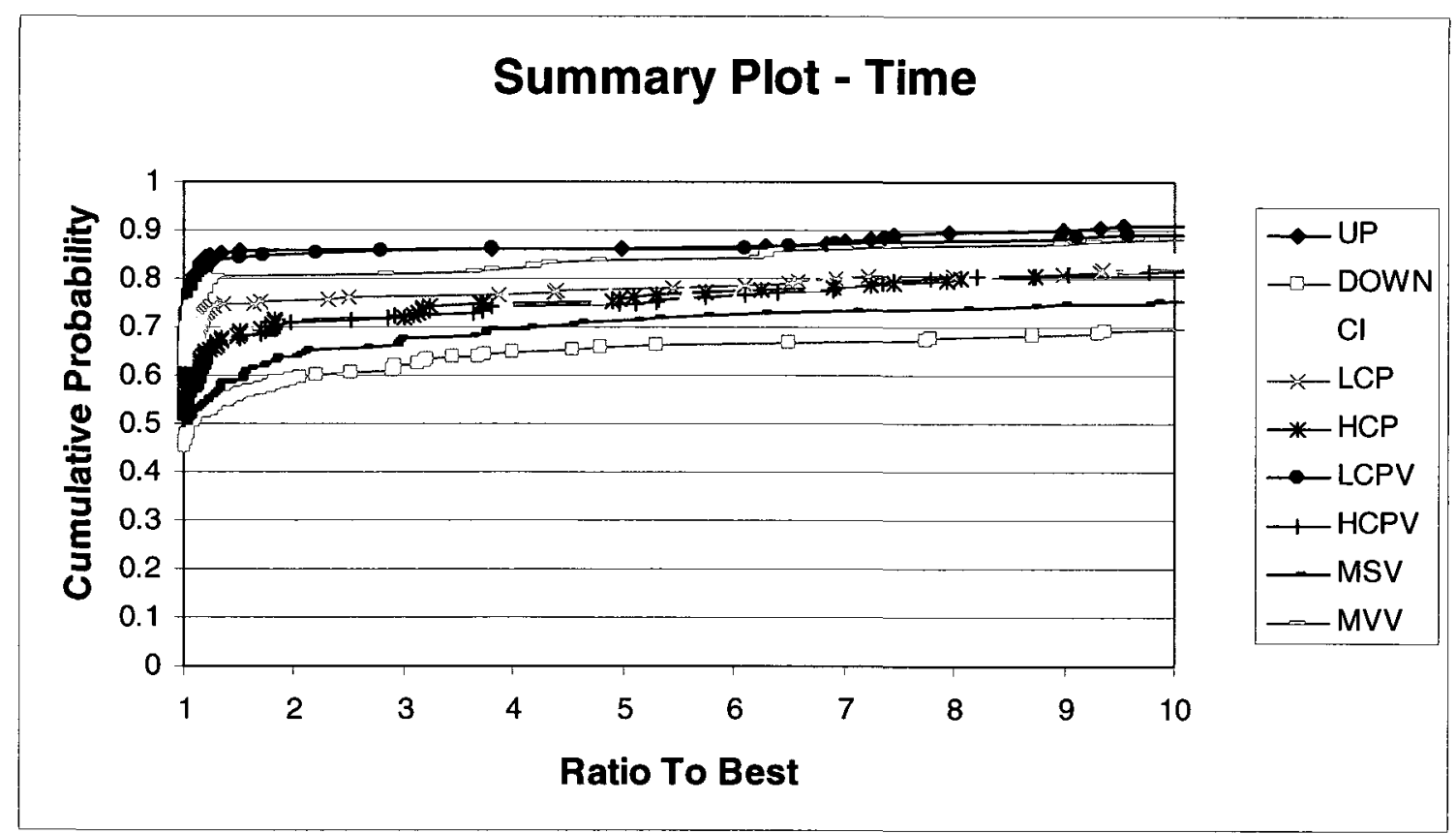

Figure 71. Experiment 1 summary plots for MIPs without multiple choice constraints computation time 


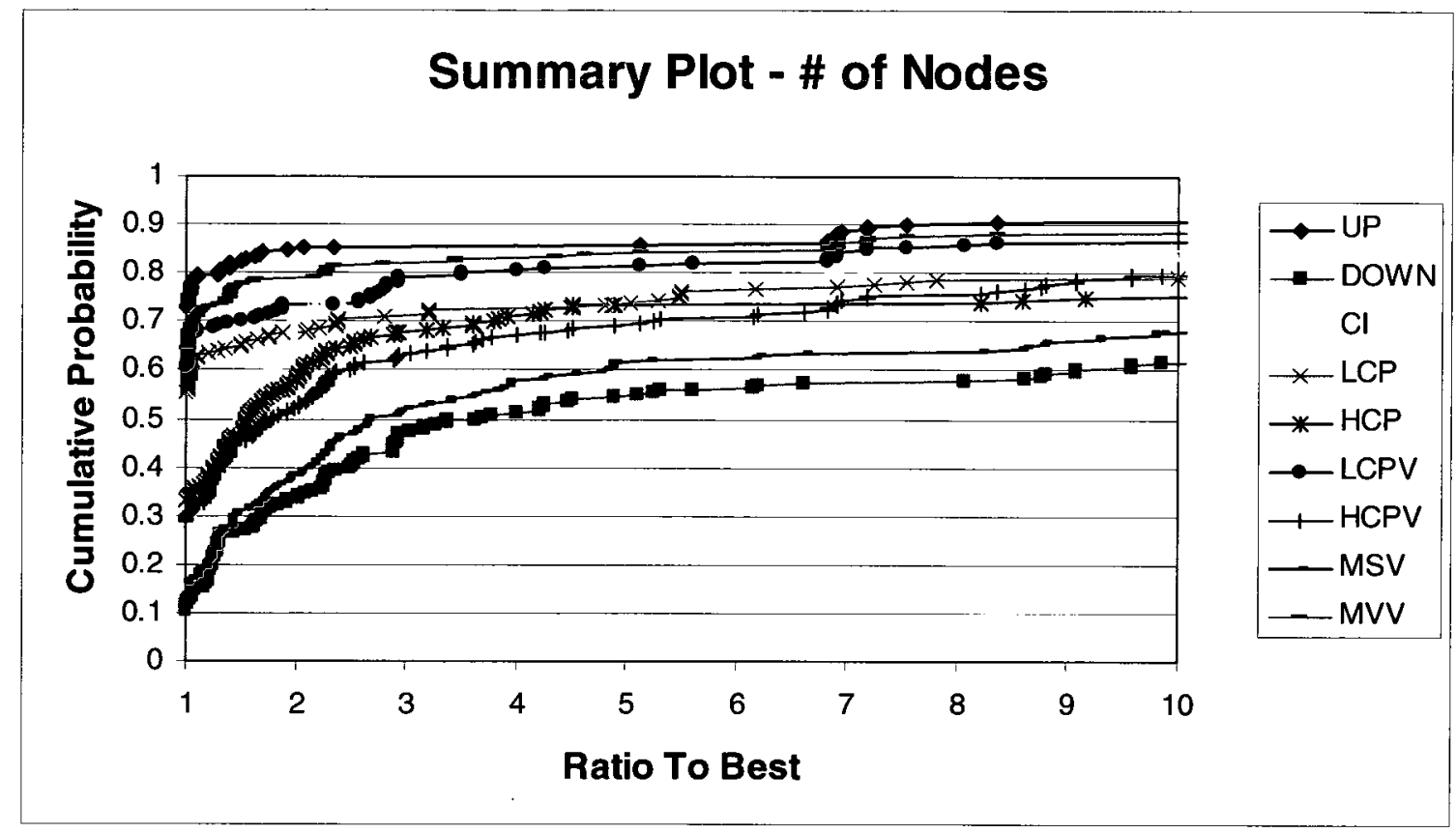

Figure 72. Experiment 1 summary plots for MIPs without multiple choice constraints number of nodes

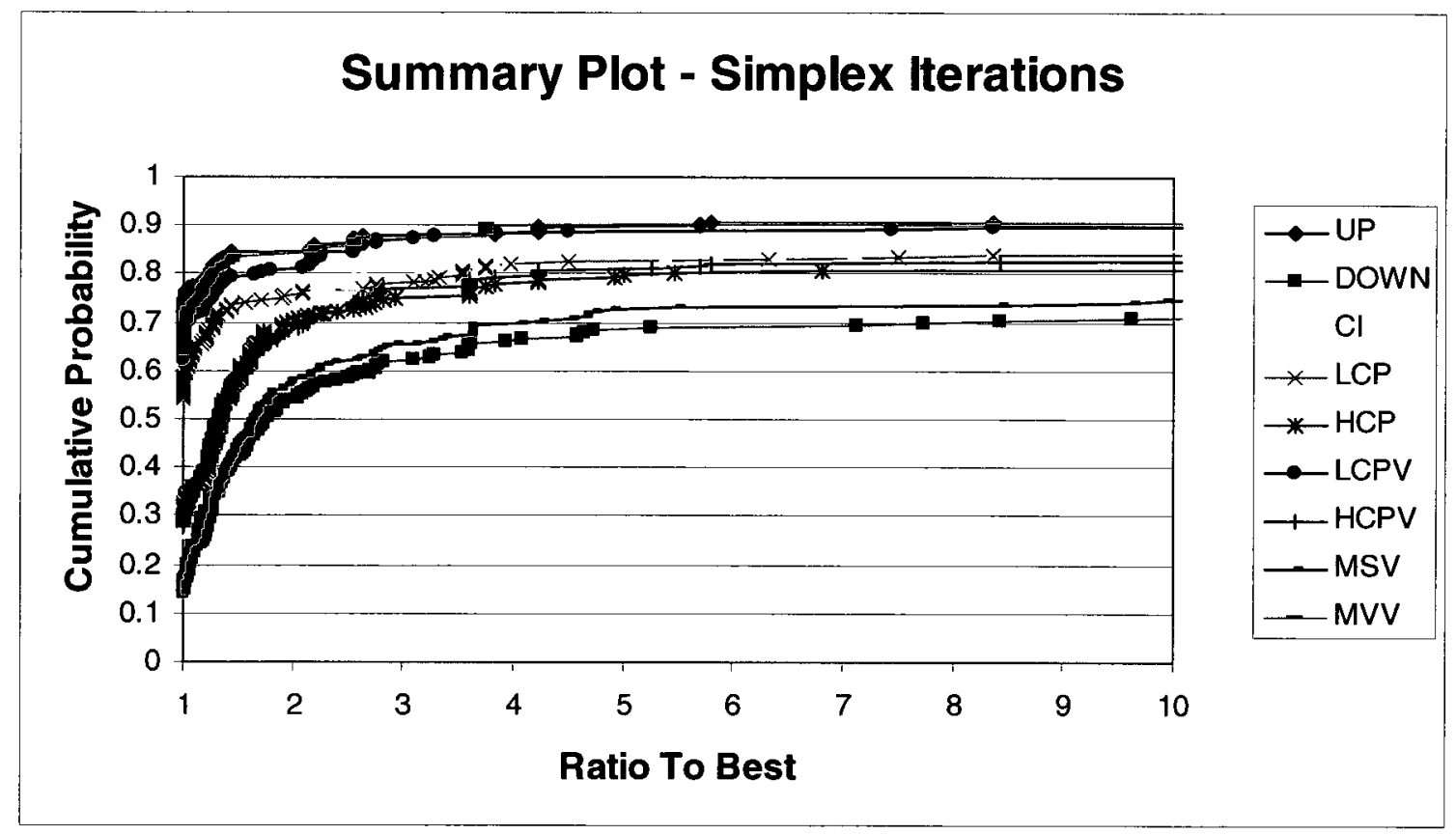

Figure 73. Experiment 1 summary plots for MIPs without multiple choice constraints simplex iterations

VDS-LCP tends to select the branching direction up when calculating a cumulative probability on multiple choice constraints. A theoretical analysis (see next 
paragraph for details) was conducted on how VDS-LCP calculates cumulative probabilities in multiple choice constraints. It shows that regardless of the initial fractional values of the variables in the multiple choice constraint, the only instance where VDS-LCP will recommend branching down is if there are two variables in an equality multiple choice constraint, and that constraint/variable combination happens to have the lowest cumulative probability out of all of the other options available. This rare set of circumstances, where VDS-LCP branches down on a multiple choice constraint, occurs in only one of the MIPs in the MIP set, cap6000.mps. Coincidentally, this MIP happens to be one of the MIPs in which VDS-LCP dominates METHA in all three performance metrics. This means that VDS-LCP is able to recognize when to branch down, as well as recognizing that it is typically more beneficial to branch up on multiple choice constraints.

The theoretical analysis is as follows: given a multiple choice constraint, the only thing that changes the cumulative probability values is the number of variables in the multiple choice constraint. The lower bound and upper bound for each variable is always 0 and 1 respectively, except for the branching variable. In the down direction the branching variable is fixed to 0 , and in the up direction the branching variable is fixed to 1. Looking at the calculation for variance, the variance will be the same value regardless of the branching direction (up or down) because the lower bound value is subtracted from the upper bound value, and $1-1$ and $0-0$ have the same value in the variance formula (Equation 2 from Section 2.2). However, the mean will have different values depending on the branching direction. In fact, branching up will always have a mean value exactly 1 higher than branching down due to the lower bound plus upper bound section in the mean 
formula (Equation 1 from Section 2.2). The values for variance and mean are given for various quantities of variables in Table 10.

Table 10. Values of mean and variance in a multiple choice constraint

\begin{tabular}{|c|c|c|c|c|}
\hline \# Variables & Mean Up & Mean Down & Variance Up & Variance Down \\
\hline 2 & 1.5 & 0.5 & 0.25 & 0.25 \\
\hline 3 & 2 & 1 & 0.5 & 0.5 \\
\hline 4 & 2.5 & 1.5 & 0.75 & 0.75 \\
\hline 5 & 3 & 2 & 1 & 1 \\
\hline 6 & 3.5 & 2.5 & 1.25 & 1.25 \\
\hline
\end{tabular}

Having these consistent differences means the focus on the analysis is how many variables are required to force the branching down probability to be lower than the branching up probability. When looking at multiple choice constraints that are less-than inequalities, the branching up cumulative probability is always less than the branching down cumulative probability (see Table 11 below). This occurs because the cumulative probability is calculated by comparing the mean to the RHS value of the constraint which is always 1 . Since the mean in the up direction is always 1 greater than the down direction, the up direction mean will always have a slightly lower cumulative probability (slightly further away from the RHS value of 1). This means that the up direction is always selected for inequality multiple choice constraints.

Table 11. Values of cumulative probabilities in a multiple choice constraint

\begin{tabular}{|c|c|c|}
\hline \# Variables & Cumulative Probability Up & Cumulative Probability Down \\
\hline 2 & 0.158655254 & 0.841344746 \\
\hline 3 & 0.078649604 & 0.5 \\
\hline 4 & 0.041632258 & 0.281851431 \\
\hline 5 & 0.022750132 & 0.158655254 \\
\hline 6 & 0.012673659 & 0.089856247 \\
\hline
\end{tabular}

However, in equality multiple choice constraints, the ratio of the two sides of the cumulative probability curves causes the case where there are two variables in the constraint to have the same ratio (refer to Section 5.1 .1 for the equality constraint ratio) 
regardless of the branching direction. This occurs because it is the only instance where the down cumulative probability is greater than 0.5 and the up probability is less than 0.5 . The values of the equality ratios are given below in Table 12 for a varying number of variables in a multiple choice constraint.

Table 12. Values of equality ratios in a multiple choice constraint

\begin{tabular}{|c|c|c|}
\hline \# Variables & Equality Ratio Up & Equality Ratio Down \\
\hline 2 & 0.188573417 & 0.188573417 \\
\hline 3 & 0.085363401 & 1 \\
\hline 4 & 0.043440797 & 0.392469529 \\
\hline 5 & 0.023279749 & 0.188573417 \\
\hline 6 & 0.012836343 & 0.098727533 \\
\hline
\end{tabular}

In the case where there are two variables in an equality multiple choice constraint, the lowest cumulative probability algorithm finds that the ratio in the down direction has a slightly smaller value. Even though in Table 12 they have the same value, the computer rounding error causes the down direction equality ratio to appear slightly smaller than the up direction. This also makes common sense because when there are two binary variables: forcing one variable to zero forces the other variable to one. Regardless of branching direction selected, the case with two binary variables forces the same changes to occur.

When looking at the cases where branch up performs poorly as an algorithm, the method that tends to dominate is the branch down heuristic. This makes intuitive sense, because if branching up leads the $B \& B$ tree down a long unfruitful path, then doing the opposite could produce a feasible solution quickly. Clearly, there are cases where branching down is the correct thing to do, and VDS-LCP is able to recognize those instances. 


\subsection{Number of Violated Constraints in MIPs}

The manner in which the MIP is formulated also has an effect on which branching direction technique is more effective. It is interesting that the violated votes method is the second best branching variable direction heuristic. For this reason, an analysis was conducted to count the number of candidate variable instances in constraints for the MIPs where branching up would force a constraint to be violated versus the number of candidate variable instances in constraints that would be violated by branching in the down direction. For example, if there are three greater-than inequality constraints in a MIP that will be violated by branching down on the integer variables $x_{1}$ or $x_{2}$ (both having positive coefficients in each of the three constraints), and one less-than inequality constraint that will be violated by branching up on the integer variables $x_{l}$ or $x_{2}$ (both having positive coefficients in the constraint), the count will be six counts for the down direction, two counts for the up direction. This analysis revealed that there are more candidate variable instances in constraints violated by branching up in the MIP set, rather than branching down. A total of 51\% of the MIPs have more candidate variable instances in constraints that would be violated by branching up (Case 1). $36 \%$ have more candidate variable instances in constraints that would be violated by branching down (Case 2), and $13 \%$ of the MIPs have an equal number of candidate variable instances in constraints that would be violated by branching up or branching down. So this could explain why the branching up method would be receiving similar results to the violated votes method. The exact counts for each MIP are presented in Appendix A.6.

Furthermore, when looking at the instances where branching up performs poorly as a heuristic, it is noted that these tend to be Case 2 MIPs, or that the MIP did not 
contain any multiple choice constraints. These two factors appear to work together to ensure that branching up works well or performs poorly. Table 13 shows the MIPs that branching up usually performed poorly on (over the six variable/node selection combinations). In this case poor performance was deemed to be a ratio to best greater than five for one of the performance metrics on at least three of the six variable/node selection combinations. In Table 13 there are only three out of eleven MIPs where branching up's poor performance is not explained by either multiple choice constraints or constraint violations.

Table 13. MIPS that branching up performed poorly on

\begin{tabular}{|c|c|c|}
\hline MIP & $\begin{array}{c}\text { No Multiple Choice } \\
\text { Constraints }\end{array}$ & $\begin{array}{c}\text { More Variables Violated when } \\
\text { Branching Down (Case 2) }\end{array}$ \\
\hline danoint.mps & Yes & Yes \\
\hline liu.mps & Yes & Tied \\
\hline momentum1.mps & No & No \\
\hline neos-1056905.mps & Yes & Tied \\
\hline neos-1122047.mps & Yes & Yes \\
\hline neos-1616732.mps & No & No \\
\hline neos-785914.mps & No & Yes \\
\hline neos-856059.mps & No & No \\
\hline neos-953928.mps & No & No \\
\hline noswot.mps & Yes & No \\
\hline rout.mps & Yes & \\
\hline
\end{tabular}

Now let's shift the focus to why the violated votes method works well. Similarly to the branch up heuristic, the violated votes method performs well when multiple choice constraints are included in the MIP. This is because the violated votes method will add votes in the up branching direction. As described earlier, branching in the up direction on multiple choice constraints forces several candidate variables to integrality simultaneously.

If this idea is then extended to general integer constraints, a similar reaction occurs. Branching in the direction that violates the constraint can force more than one 
candidate variable simultaneously to integrality; whereas branching in the direction that satisfies the constraint allows for situations where no candidate variable, other than the branching variable, has to change its value.

In particular consider the active constraint below:

$$
x_{1}+x_{2}+x_{3} \leq 3
$$

where the allowable range of each integer variable is $[0,2]$ and the current LP relaxation values of the variables are $(0.5,1.6,0.9)$ respectively. If we branch up on $x_{3}$ (in the violated direction), we might change the variable values to $(0.5,1.5,1)$. Although the variables are not forced to integrality in this case, some are forced to change to another value. This is still good because we are at least forcing change, and that change could force a non-integer value to an integer. Whereas branching in the satisfied direction does not force the other variables to change their values (the variable values sum to less-than three). The forced change happens when the constraint has positive coefficients, is a lessthan inequality, and therefore the violating direction is up.

To reiterate, the violated votes method works because it forces more than one variable to adjust at once; no other variable has to change value if you branch in the satisfied direction. Hence it is possible that multiple integer and/or binary variables will become integer at the same time. This is an absolute certainty in multiple choice constraints, and more like a heuristic probability in the case of general integers. In addition, because of the make-up of the constraints in the MIPs, the violated votes method tends to vote in the branching up direction more often. This helps explain why the branch up method performs well: because it forces several candidate variables to 
adjust at once. It should be noted that these same principles hold in inequalities whose variables are a mix of positive and negative coefficients.

\subsection{Focusing on Hardest Constraints}

As mentioned in Section 9.1, VDS-LCP tends to branch up when selecting its branching direction on multiple choice constraints, and yet it also selects branching down in instances where it is beneficial to do so. To further the argument that VDS-LCP branches down in smart instances, another analysis of the results was performed. Here, the MIPs in which VDS-LCP dominates the performance metrics are considered, regardless of their MIP characteristics. On seven MIPs VDS-LCP dominates METHA in terms of performance. In all seven of these MIPs, VDS-LCP branches down at some point in the course of the B\&B tree. It does not branch down continuously, but all of the seven models have at least one instance where the down branch is followed. VDS-LCP is branching down in these instances because it is looking at the lowest cumulative probability for any candidate variable/constraint combination. Essentially, it is picking the hardest constraint to satisfy, and resolving it first.

Consider a large group meeting that is being organized at a company. Each person attending the meeting has their own level of availability. Employees with a lower ranking have fewer other engagements to attend, whereas the CEO has a very busy schedule and it is difficult to get a meeting with them. If an algorithm were applied to scheduling this meeting and each person attending was asked individually when they could attend the meeting, the best way to select a meeting time in which every person could attend is to start with the busiest individual first. By knowing their schedule and fitting all of the less 
busy people into the busiest person's time slot, there are not only fewer options for the group in terms of meeting times, but there are more chances of satisfying everyone's schedules because the hardest person to schedule has already been accommodated. In terms of a scheduling algorithm, VDS-LCP is scheduling the busiest person first by selecting the lowest cumulative probability. This is another reason why VDS-LCP performs well as a heuristic: it focuses on and satisfies the hardest constraints in the MIP first.

The smart branching down pattern applies especially when VDS-LCP finds an equality constraint to have the lowest probability. All cases where VDS-LCP branches on an equality constraint and branches down resulted in a reaching a feasible solution quicker (better on all three performance measures) than METHA. However, VDS-LCP hardly ever branches on an equality constraint (only $17 \%$ of the time when it has both inequality and equality constraints to choose from). Equality constraints are always active, and so in METHA a candidate variable can look like a great candidate if it in many equality constraints. VDS-LCP does not recognize these candidate variables as being special. It continues to focus on the absolute lowest probability, or the constraint that has the worst chance of ever being satisfied, and gives it priority. By solving these hardest constraints first, it works toward guaranteeing a feasible solution in the future. So the main difference between METHA and VDS-LCP on the equality-containing MIPs is that METHA focuses on candidate variables in equalities, and VDS-LCP focuses on the overall hardest constraint. VDS-LCP is able to identify when an equality constraint and branching variable combination should take precedence in a MIP with both inequality and equality constraints. 
HYBRID2 (refer to Section 5.3.4) confirms the above theory. This experimental run is conducted only on the equality-containing MIP set, because the equality-free MIPs use METHA at every node for this hybrid. When HYBRID2 is compared to METHA (see the three figures below) the hybrid outperforms METHA on all performance metrics and it slightly beats VDS-LCP in terms of time and number of nodes visited, while tying in robustness and number of simplex iterations.

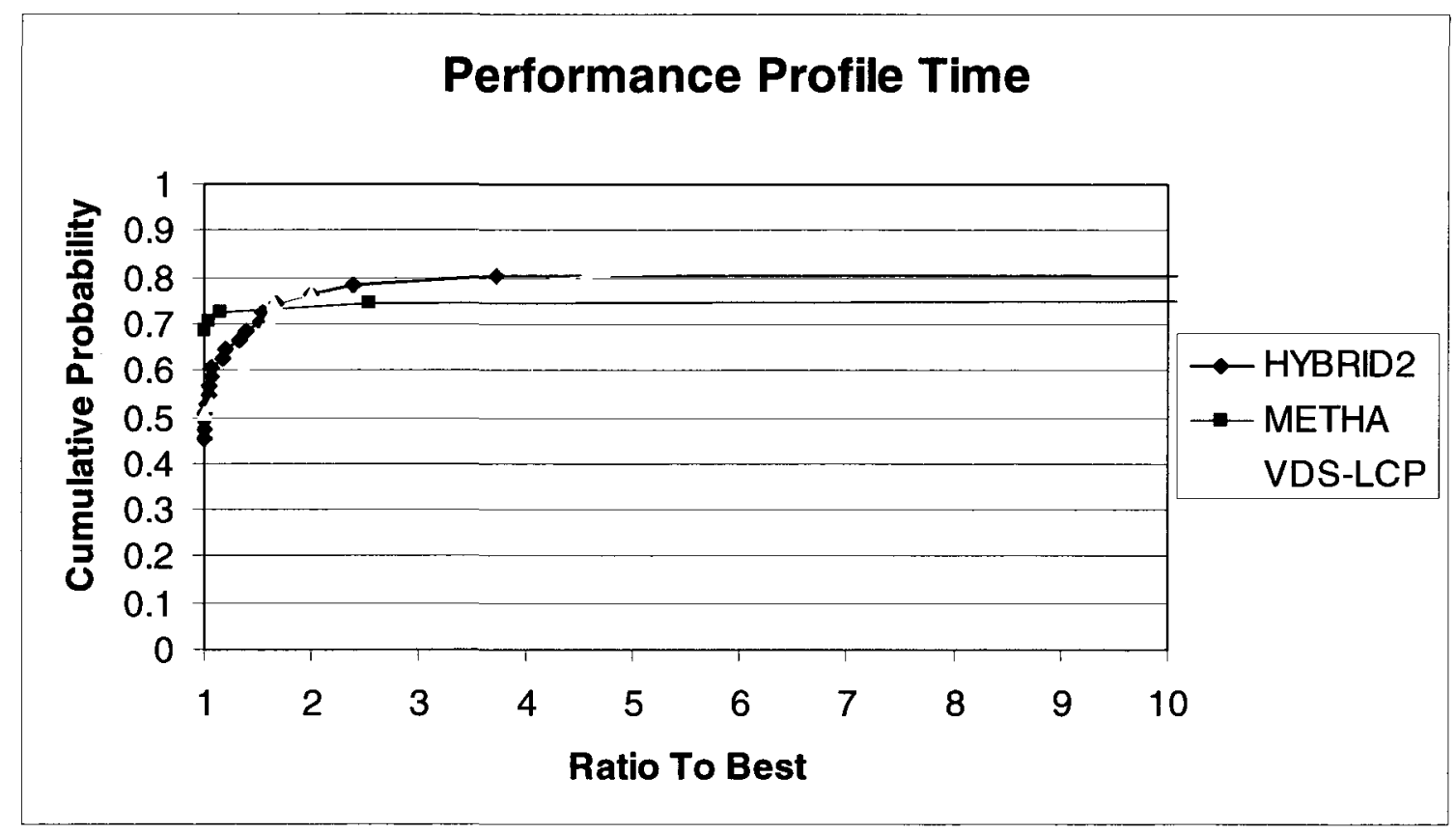

Figure 74. Experiment 2 performance profile for HYBRID2 - computation time 


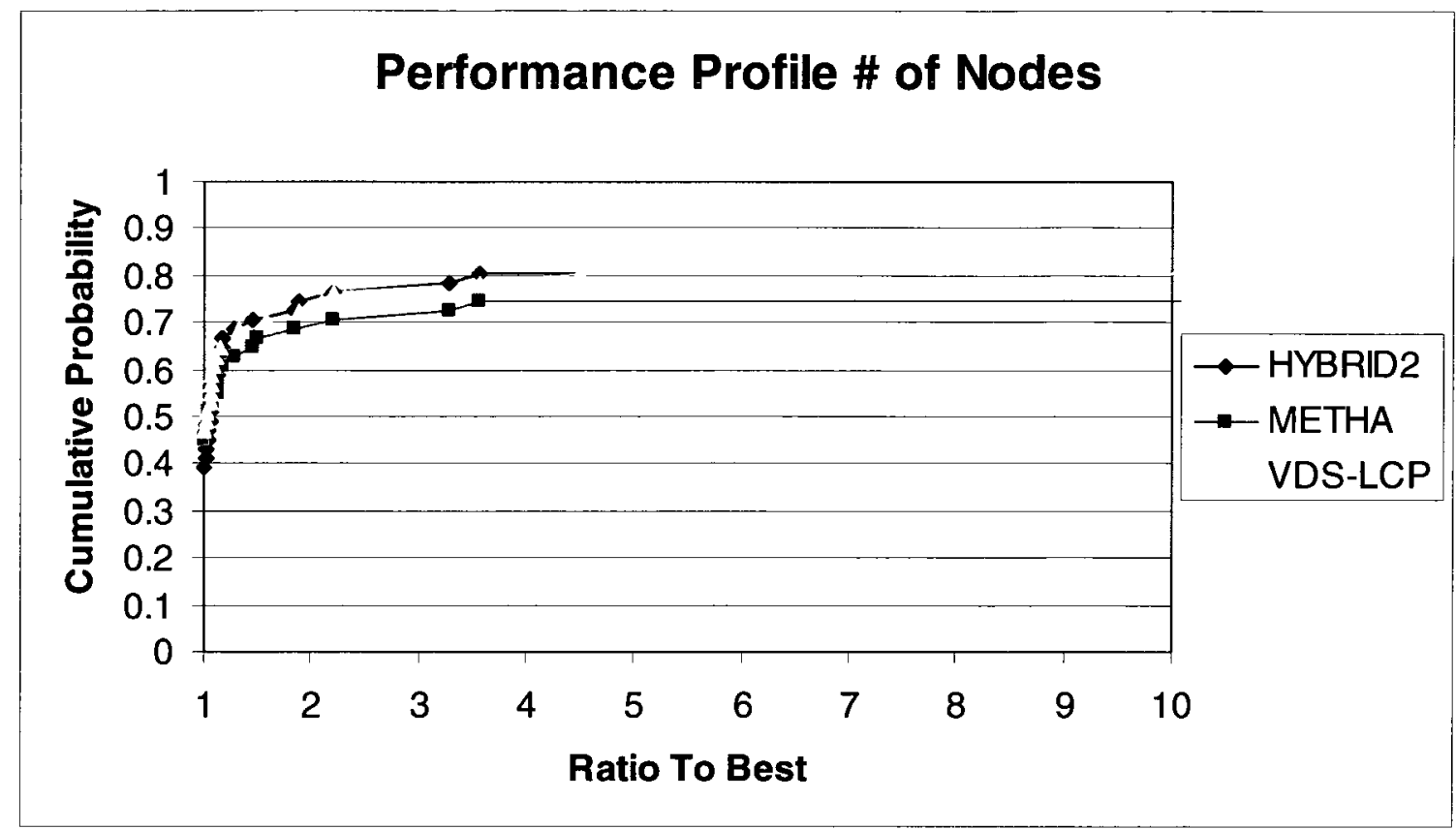

Figure 75. Experiment 2 performance profile for HYBRID2 - number of nodes

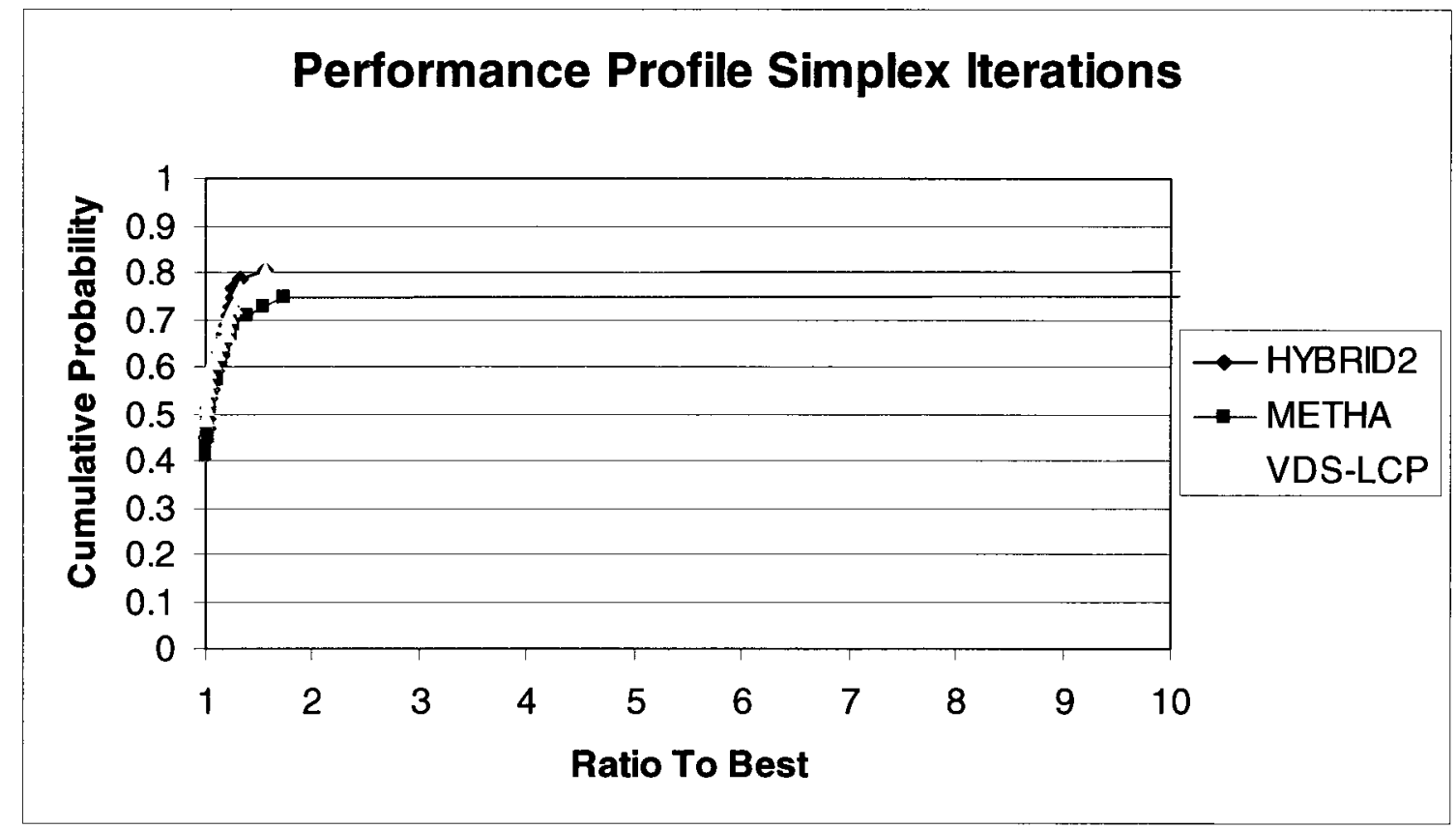

Figure 76. Experiment 2 performance profile for HYBRID2 - simplex iterations

VDS-LCP does well in situations of mixed constraints, because it gives equal focus to inequality and equality constraints as potential hard constraints to satisfy. It then satisfies the most difficult constraint. Whereas METHA performs better when there are 
only equality constraints or only inequality constraints with candidate variables, because it over-inflates the importance of equality constraints (they are always active).

To reiterate, the data shows that VDS-LCP works well on MIPs with equality constraints because it performs smart branches down, and it doesn't over-inflate the importance of the equality constraints (since they are always active). VDS-LCP focuses instead on the overall hardest constraint to satisfy in the entire MIP based on the allowable ranges for all of the variables in the constraint. 


\section{Conclusions}

\subsection{Conclusions}

The following is a summary of the conclusions made by this research:

- The branching direction heuristic has a significant impact on the solution speed.

- Branching up works well regardless of the variable selection or node selection policy.

- When branching up performs poorly, branching down tends to perform well.

- Branching in the direction that violates a constraint is able to simultaneously force more candidate variables to integrality.

- Branching up is a good branching variable direction selection policy due to the role of multiple choice constraints in MIPs as well as the general formulation of the MIPs, which tend to have more constraints that are violated by branching up. Thus branching up is more often able to force more than one candidate variable to become integer-valued simultaneously.

- There are often numerous ties for the branching variable. Looking at a common subset of ties amongst one or more variable selection methods can produce an equal or slightly better result.

- The lowest cumulative probability variable and direction selection method performs differently depending on the types of constraints in the MIP (equalityfree versus equality-containing). It tends to perform better on equality-containing MIPs. It performs well on this class of problems because it places equal emphasis 
on equality and inequality constraints while searching to satisfy the hardest constraint/variable combination first. It schedules the busiest person first.

- The lowest cumulative probability variable and direction selection method chooses to branch in the up direction on multiple choice constraints unless there are only two variables in the multiple choice constraint (in which case it branches down).

\subsection{Summary of Contributions}

The following is a summary of the contributions to knowledge made by this research:

- Performed the first empirical evaluation of branching variable direction selection heuristics.

- Empirically verified that branching up is the best general branching variable direction selection policy.

- Developed a new method (MVV) that selects the branching variable direction and performs equal to branching up for a class of MIPs that contain at least one equality constraint.

- Developed two new methods (VDS-LCP and HYBRID2) that pick both the branching variable and branching direction simultaneously that are better than the standard GLPK 4.28 methods, and are equal to the state of the art activeconstraint variable selection Method A for a class of MIPs that contain at least one equality constraint.

- Developed four new methods (HYBRID1, TIES, VDS-LCP-UP1, and VDS-LCPUP2) that pick both the branching variable and branching direction 
simultaneously that are equal to the state of the art active-constraint variable selection Method A.

- Extended the probability methods to continuous constraints.

- Extended the probability methods to handle equality constraints via a ratio that gauges the centeredness of the equality constraint.

- Identified the importance of branching in a direction that violates a constraint to force as many candidate variables as possible to change their values. This is the principle behind both MVV and LCP.

\subsection{Future Research}

The following is a list of future research that could be conducted to further the contributions of this thesis:

- Explore the idea of branching on the active constraint with the most candidate variables and branching to the violated side. This idea is based on the fact that branching to the violated side of the constraint forces multiple candidates to change at once. So if a constraint contained a large number of candidates, there is a good chance that many of them could be removed from the candidates list if a decision were made that forced them to integrality.

- Explore the idea of branching on the candidate variable and direction that forces the most other candidate variables to change values. "Most" is based on the number of distinct candidate variables present in active constraints that would be violated by branching in the direction of the proposed candidate variable. Again, 
forcing multiple candidate variables to change at once gives more opportunities for multiple candidate variables to become integer-valued.

- Explore the probability methods when only cycling through the active constraints at that node. Currently the probability methods look at each constraint/candidate variable combination. However, as shown in the active-constraint variable selection theory [Patel and Chinneck 2007], making changes to the active constraints produces two child nodes that would be far apart in terms of their respective bounding function values. One of these nodes would hopefully never have to be expanded, thus reducing the size of the search tree. 


\section{References}

Abramowitz, M., and Stegun, I.A., eds., Handbook of Mathematical Functions, National Bureau of Standards, Applied Mathematics Series, 1965, pp. 932.

Achterberg, T., Koch, T., and Martin, A., “Branching Rules Revisited”, Operations Research Letters, Volume 33, Issue 1, Berlin, Germany, January 2005, pp. 42-54.

Achterberg, T., Koch, T., and Martin, A., "MIPLIB 2003”, Operations Research Letters, Volume 34, Issue 4, Berlin Germany, 2006, pp. 1-12.

Bernatzki, K.P., Bussieck, M.R., Lindner, T., and Lübbecke, M.E., “Optimal Scrap Combination for Steel Production”, OR Spektrum, No 20, 1998, pp. 251-258.

Bixby, R.E., Boyd, E.A., and Indovina, R.R., "MIPLIB: A Test Set of Mixed Integer Programming Problems”, SIAM News, Volume 25, Issue 2, March 1992.

Bixby, R.E., Ceria, S., McZeal, C.M., Savelsbergh, M.W.P., “An Updated Mixed Integer Programming Library: MIPLIB 3.0”, Optima, Volume 58, 1998, pp. 12-15.

Brooks, D.R., C Programming: The Essentials for Engineers and Scientists, Springer, 1998, pp. 116.

Ching-Jong, L., "A New Node Selection Strategy in the Branch-and-Bound Procedure", Computers \& Operations Research, Volume 21, Issue 10, December 1994, pp. 10951101.

Dolan, E.D., and Moré, J., "Benchmarking Optimization Software with Performance Profiles”, Mathematical Programming, Series A, Volume 91, 2002, pp. 201-213.

Driebeek, N.J., “An Algorithm for the Solution of Mixed Integer Programming Problems”, Management Science, Volume 12, 1966, pp. 576-587.

Jariwala, A., "Efficient Branch and Bound Algorithm for the Dynamic Layout Problem", Master's Thesis, Ohio University, Industrial and Manufacturing Systems Engineering, 1995.

Laplace, C., Lai, H., Khan, M., Van der Heidjen, J.J., and Hendrix, C., Dev-C++ 4, GNU General Public License, http://www.bloodshed.net/devcpp.html, [Accessed May 2009].

Linderoth, J., Coral: Mixed Integer Programming Instances, http://coral.ie.lehigh.edu/mip-instances/, [Accessed May 2009]. 
Linderoth, J.T., and Savelsbergh, M.W.P., "A Computational Study of Search Strategies for Mixed Integer Programming", INFORMS Journal of Computing, Volume 11, Issue 2, February 1999, pp. 173-187.

Linderoth, J., and Ralphs, T., "Noncommercial Software for Mixed-Integer Linear Programming", Technical Report 04T-023, Department of Industrial and Systems Eng., Lehigh Univ., Dec 2004. www.lehigh.edu/ jt13/teaching/ie418/papers/MILP04.pdf.

Makhorin, A., "GNU Linear Programming Kit Reference Manual Version 4.28", 2008, http://www.gnu.org/software/glpk/, [Accessed May 2009].

Meyer, R.R., D'Souza,W.D., Ferris, M.C., and Thomadsen, B.R., "MIP Models and BB Strategies in Brachytherapy Treatment Optimization", Journal of Global Optimization, Volume 25, 2003, pp. 23-42.

Patel, J., and Chinneck, J.W., "Active-Constraint Variable Ordering for Faster Feasibility of Mixed Integer Linear Programs", Mathematical Programming, Series A, Volume 110, 2007, pp. 445-474.

Pesant, G., and Quimper, C.G., "Counting Solutions of Knapsack Constraints", CPAIOR 2008, LNCS 5015, 2008, pp. 203-217.

Tomlin, J.A., "An Improved Branch-and-Bound Method for Integer Programming", Operations Research, Volume 19, 1971, pp. 1070-1075.

Winston, W.L., Operations Research Applications and Algorithms: Fourth Edition, Thomason Brooks/Cole, California, 2004, pp. 475-477, 512-524. 


\section{Appendix A}

\section{A.1 Pseudocode for Other Cumulative Probability Direction Selection Heuristics}

See below for the pseudocode for the highest cumulative probability direction selection heuristic. That is followed by the lowest cumulative probability votes direction selection heuristic and the highest cumulative probability votes direction selection heuristic.

Input: MIP; candidate variable selected for branching called candidate.

0. Initialize: HighestProb $\leftarrow-0.1$; Direction $\leftarrow \phi$

1. For each constraint the candidate variable is present in:

1.1 GetProbabilities(candidate, constraint)

1.2 If it is a $\geq$ constraint:

$$
\text { 1.2.1 ProbUp } \leftarrow 1-\text { ProbUp; ProbDown } \leftarrow 1-\text { ProbDown }
$$

1.3 If it is an = constraint:

1.3.1 If ProbUp > 1 - ProbUp:

1.3.1.1 ProbUp $\leftarrow(1-$ ProbUp $) /$ ProbUp

1.3.2 Else: ProbUp $\leftarrow$ ProbUp / $(1-$ ProbUp $)$

1.3.3 If ProbDown > 1 - ProbDown:

2.9.3.1 ProbDown $\leftarrow(1-$ ProbDown $) /$ ProbDown

1.3.4 Else: ProbDown $\leftarrow$ ProbDown / (1 - ProbDown $)$

1.4 Check probabilities (up and down) against current highest

1.4.1 If ProbUp > HighestProb:

1.4.1.1 HighestProb $\leftarrow$ ProbUp

1.4.1.2 Direction $\leftarrow$ up

1.4.2 If ProbDown > HighestProb:

1.4.2.1 HighestProb $\leftarrow$ ProbDown

1.4.2.2 Direction $\leftarrow$ down

2. Branch in direction Direction. 
Lowest Cumulative Probability by Votes Branching Direction Method

Input: MIP; candidate variable selected for branching called candidate.

0 . Initialize: LowestProb $\leftarrow 1.1$; Direction $\leftarrow \phi ;$ TallyUp $\leftarrow 0$; TallyDown $\leftarrow 0$;

1. For each constraint the candidate variable is present in:

1.1 GetProbabilities(candidate, constraint)

1.2 If it is a $\geq$ constraint:

1.2.1 ProbUp $\leftarrow 1-$ ProbUp; ProbDown $\leftarrow 1-$ ProbDown

1.3 If it is an $=$ constraint:

1.3.1 If ProbUp > 1-ProbUp:

1.3.1.1 ProbUp $\leftarrow(1-$ ProbUp $) /$ ProbUp

1.3.2 Else: ProbUp $\leftarrow$ ProbUp / (1 - ProbUp $)$

1.3.3 If ProbDown > 1 - ProbDown:

1.3.3.1 ProbDown $\leftarrow(1-$ ProbDown $) /$ ProbDown

1.3.4 Else: ProbDown $\leftarrow$ ProbDown / (1 - ProbDown $)$

1.4 Compare up and down probabilities, increment tallies accordingly

1.4.1 If ProbDown < ProbUp:

1.4.1.1 Increment TallyDown

1.4.2 Else: Increment Tally $U p$

2. If TallyDown > TallyUp:

2.1 Direction $\leftarrow$ down

3. Else: Direction $\leftarrow$ up

4. Branch in direction Direction. 
Highest Cumulative Probability by Votes Branching Direction Method

Input: MIP; candidate variable selected for branching called candidate.

0 . Initialize: HighestProb $\leftarrow-0.1$; Direction $\leftarrow \phi$; Tally $U p \leftarrow 0$; TallyDown $\leftarrow 0$;

1. For each constraint the candidate variable is present in:

1.1 GetProbabilities(candidate, constraint)

1.2 If it is a $\geq$ constraint:

1.2.1 ProbUp $\leftarrow 1-$ ProbUp; ProbDown $\leftarrow 1-$ ProbDown

1.3 If it is an = constraint:

1.3.1 If ProbUp > 1 - ProbUp:

1.3.1.1 ProbUp $\leftarrow(1-$ ProbUp $) /$ ProbUp

1.3.2 Else: ProbUp $\leftarrow$ ProbUp / (1 - ProbUp $)$

1.3.3 If ProbDown > $1-$ ProbDown:

1.3.3.1 ProbDown $\leftarrow(1-$ ProbDown $) /$ ProbDown

1.3.4 Else: ProbDown $\leftarrow$ ProbDown / (1 - ProbDown)

1.4 Compare up and down probabilities, increment tallies accordingly

1.4.1 If ProbDown > ProbUp:

1.4.1.1 Increment TallyDown

1.4.2 Else: Increment Tally $U p$

2. If TallyDown > TallyUp:

2.1 Direction $\leftarrow$ down

3. Else: Direction $\leftarrow$ up

4. Branch in direction Direction. 


\section{A.2 Satisfied Votes Branching Direction Heuristic Pseudocode}

Similar to the violated votes pseudocode, see below for the satisfied votes pseudocode for selection a branching direction given the branching variable.

Input: MIP; candidate variable selected for branching called candidate.

0 . Initialize: Tally $U p \leftarrow 0$; TallyDown $\leftarrow 0$;

1. For each constraint the candidate is present in:

1.1 If constraint is active:

1.1.1 coeff $\leftarrow$ coefficient for candidate in constraint

1.1.2 If constraint is an equality:

1.1.2.1 GetProbabilities(candidate, constraint)

1.1.2.2 If ProbDown >0.5:

1.1.2.2.1 ProbDown $\leftarrow(1-$ ProbDown $)$

1.1.2.3 If ProbUp >0.5:

1.1.2.3.1 ProbUp $\leftarrow(1-$ ProbUp $)$

1.1.2.4 If ProbDown > ProbUp:

1.1.2.4.1 Increment TallyDown

1.1.2.5. Else: Increment Tally $U p$

1.1.3 Else if constraint is a less-than inequality:

1.1.3.1 If coeff is positive:

1.1.3.1.1 Increment TallyDown

1.1.3.2 Else: Increment TallyUp

1.1.4 Else:

1.1.4.1 If coeff is positive:

1.1.4.1.1 Increment Tally $U p$

1.1.4.2 Else: Increment TallyDown

2. If TallyDown is larger than TallyUp: branch down

3. Else: branch up. 


\section{A.3 List of Equality-Containing MIPs}

Below is the list of the 51 equality-containing MIPs used in the experiments. Each MIP specifies the number of variables, the number of constraints, and whether the MIP contained any multiple choice constraints or not.

\begin{tabular}{|l|r|r|l|}
\hline MIP & \# Constraints & \# Variables & Multiple Choice Constraints? \\
\hline 10teams.mps & 230 & 2025 & yes \\
\hline a1c1s1.mps & 3312 & 3648 & no \\
\hline aflow30a.mps & 479 & 842 & yes \\
\hline aflow40b.mps & 1442 & 2728 & yes \\
\hline air04.mps & 823 & 8904 & yes \\
\hline air05.mps & 426 & 7195 & yes \\
\hline arki001.mps & 1048 & 1388 & yes \\
\hline atlanta-ip.mps & 21732 & 48738 & yes \\
\hline cap6000.mps & 2176 & 6000 & yes \\
\hline dano3mip.mps & 3202 & 13873 & no \\
\hline danoint.mps & 664 & 521 & no \\
\hline disktom.mps & 399 & 10000 & yes \\
\hline ds.mps & 656 & 67732 & yes \\
\hline fiber.mps & 363 & 1298 & yes \\
\hline fixnet6.mps & 478 & 878 & no \\
\hline gesa2.mps & 1392 & 1224 & yes \\
\hline gesa2-o.mps & 1248 & 1224 & yes \\
\hline glass4.mps & 396 & 322 & yes \\
\hline harp2.mps & 112 & 2993 & yes \\
\hline markshare1.mps & 6 & 62 & no \\
\hline markshare2.mps & 136 & 240 & no \\
\hline misc07.mps & 246 & 240 & no \\
\hline mkc.mps & 212 & 260 & yes \\
\hline mod011.mps & 3411 & 5325 & yes \\
\hline modglob.mps & 4480 & 10958 & no \\
\hline momentum1.mps & 291 & 422 & no \\
\hline momentum2.mps & 42680 & 5174 & yes \\
\hline momentum3.mps & 24237 & 3732 & yes \\
\hline msc98-ip.mps & 56822 & 13532 & yes \\
\hline mzzv11.mps & 15850 & 21143 & yes \\
\hline mzzv42z.mps & 9499 & 10240 & yes \\
\hline net12.mps & 10460 & 11717 & yes \\
\hline noswot.mps & 14021 & 14115 & yes \\
\hline nw04.mps & 182 & 128 & no \\
\hline opt1217.mps & 36 & 7672 & yes \\
\hline pk1.mps & & yes \\
\hline pp08a.mps & & 86 & no \\
\hline pp08aCUTS.mps & & & \\
\hline
\end{tabular}




\begin{tabular}{|l|r|r|l|}
\hline protfold.mps & 2112 & 1835 & yes \\
\hline qiu.mps & 1192 & 840 & no \\
\hline rd-rplusc-21.mps & 125899 & 622 & yes \\
\hline roll3000.mps & 2295 & 1166 & yes \\
\hline rout.mps & 291 & 556 & yes \\
\hline set1ch.mps & 492 & 712 & no \\
\hline stp3d.mps & 159488 & 204880 & yes \\
\hline swath.mps & 884 & 6805 & yes \\
\hline t1717.mps & 551 & 73885 & yes \\
\hline timtab1.mps & 171 & 397 & no \\
\hline timtab2.mps & 294 & 675 & no \\
\hline tr12-30.mps & 750 & 1080 & no \\
\hline vpm2.mps & 234 & 378 & no \\
\hline
\end{tabular}




\section{A.4 List of Equality-Free MIPs}

Below is the list of the 95 equality-free MIPs used in the experiments. Each MIP specifies the number of variables, the number of constraints, and whether the MIP contained any multiple choice constraints or not.

\begin{tabular}{|l|r|r|l|}
\hline MIP & \# Constraints & \# Variables & Multiple Choice Constraints? \\
\hline bell3a.mps & 123 & 133 & yes \\
\hline bell3b.mps & 123 & 133 & yes \\
\hline bell4.mps & 105 & 117 & yes \\
\hline bell5.mps & 91 & 104 & yes \\
\hline bm23.mps & 20 & 27 & yes \\
\hline fast0507.mps & 507 & 63009 & yes \\
\hline gt2.mps & 29 & 188 & yes \\
\hline liu.mps & 2178 & 1156 & no \\
\hline Iseu.mps & 28 & 89 & yes \\
\hline manna81.mps & 6480 & 3321 & no \\
\hline mas74.mps & 13 & 151 & no \\
\hline mas76.mps & 12 & 151 & no \\
\hline mod008.mps & 6 & 319 & no \\
\hline neos-1056905.mps & 900 & 463 & no \\
\hline neos-1122047.mps & 57791 & 5100 & no \\
\hline neos-1171448.mps & 13206 & 4914 & yes \\
\hline neos-1171692.mps & 4239 & 1638 & yes \\
\hline neos-1171737.mps & 4179 & 2340 & yes \\
\hline neos-1200887.mps & 633 & 234 & yes \\
\hline neos-1211578.mps & 356 & 260 & yes \\
\hline neos-1228986.mps & 356 & 260 & yes \\
\hline neos13.mps & 20852 & 1827 & no \\
\hline neos-1311124.mps & 1643 & 1092 & yes \\
\hline neos-1337489.mps & 356 & 260 & yes \\
\hline neos-1346382.mps & 796 & 520 & yes \\
\hline neos-1420205.mps & 383 & 231 & yes \\
\hline neos-1426635.mps & 796 & 520 & yes \\
\hline neos-1426662.mps & 1914 & 832 & yes \\
\hline neos-1427181.mps & 1786 & 832 & yes \\
\hline neos-1427261.mps & 2226 & 1040 & yes \\
\hline neos-1429185.mps & 1346 & 624 & yes \\
\hline neos-1429461.mps & 1096 & 520 & yes \\
\hline neos-1430701.mps & 668 & 312 & yes \\
\hline neos-1436709.mps & 1417 & 676 & yes \\
\hline neos-1436713.mps & 2666 & 1248 & yes \\
\hline neos-1439395.mps & 364 & yes \\
\hline neos-1440447.mps & 260 & yes \\
\hline neos-1440457.mps & 936 & yes \\
\hline
\end{tabular}




\begin{tabular}{|c|c|c|c|}
\hline neos-1440460.mps & 989 & 468 & yes \\
\hline neos-1442119.mps & 1524 & 728 & yes \\
\hline neos-1442657.mps & 1310 & 624 & yes \\
\hline neos-1467067.mps & 1084 & 1196 & yes \\
\hline neos-1480121.mps & 363 & 222 & no \\
\hline neos-1516309.mps & 489 & 4500 & yes \\
\hline neos-1599274.mps & 1237 & 4500 & yes \\
\hline neos-1616732.mps & 1999 & 200 & yes \\
\hline neos-495307.mps & 3 & 9423 & no \\
\hline neos5.mps & 63 & 63 & no \\
\hline neos-544324.mps & 732 & 10080 & yes \\
\hline neos-547911.mps & 693 & 3528 & yes \\
\hline neos-565672.mps & 318334 & 190589 & yes \\
\hline neos-702280.mps & 1600 & 7199 & no \\
\hline neos-785899.mps & 1653 & 1320 & yes \\
\hline neos-785912.mps & 1714 & 1380 & yes \\
\hline neos-785914.mps & 1590 & 1260 & yes \\
\hline neos-787933.mps & 1897 & 236376 & no \\
\hline neos-841664.mps & 3135 & 2925 & no \\
\hline neos-856059.mps & 17827 & 450 & yes \\
\hline neos-872648.mps & 93291 & 175219 & no \\
\hline neos-873061.mps & 93360 & 175288 & no \\
\hline neos-885086.mps & 11574 & 4860 & yes \\
\hline neos-885524.mps & 65 & 91670 & no \\
\hline neos-886822.mps & 1089 & 1057 & no \\
\hline neos-932721.mps & 18085 & 22266 & yes \\
\hline neos-932816.mps & 30823 & 21007 & yes \\
\hline neos-933638.mps & 13658 & 32417 & yes \\
\hline neos-933966.mps & 12047 & 31762 & yes \\
\hline neos-934278.mps & 11495 & 23123 & yes \\
\hline neos-934441.mps & 11691 & 23362 & yes \\
\hline neos-948346.mps & 1570 & 57855 & yes \\
\hline neos-953928.mps & 12498 & 23305 & yes \\
\hline neos-954925.mps & 2989 & 84718 & yes \\
\hline neos-956971.mps & 2527 & 57756 & yes \\
\hline neos-957143.mps & 2767 & 57756 & yes \\
\hline neos-957323.mps & 3757 & 57756 & yes \\
\hline nsrand-ipx.mps & 735 & 6621 & yes \\
\hline p0033.mps & 16 & 33 & yes \\
\hline p0040.mps & 23 & 40 & yes \\
\hline p0201.mps & 133 & 201 & yes \\
\hline p0282.mps & 241 & 282 & yes \\
\hline p0291.mps & 252 & 291 & yes \\
\hline p0548.mps & 176 & 548 & yes \\
\hline p2756.mps & 755 & 2756 & yes \\
\hline ramos3.mps & 2187 & 2187 & no \\
\hline sentoy.mps & 30 & 60 & no \\
\hline $\begin{array}{l}\text { seymour.disj- } \\
\text { 10.mps }\end{array}$ & 5108 & 1209 & yes \\
\hline
\end{tabular}




\begin{tabular}{|l|r|r|l|}
\hline seymour.mps & 4944 & 1372 & yes \\
\hline sp97ar.mps & 1761 & 14101 & yes \\
\hline sp97ic.mps & 2086 & 1662 & yes \\
\hline sp98ar.mps & 4680 & 5478 & yes \\
\hline sp98ic.mps & 2311 & 2508 & yes \\
\hline sp98ir.mps & 1531 & 1680 & yes \\
\hline stein15.mps & 36 & 15 & yes \\
\hline stein27.mps & 118 & 27 & yes \\
\hline stein45.mps & 331 & 45 & yes \\
\hline
\end{tabular}




\section{A.5 List of MIPs Where VDS-LCP and METHA Do Not Both Time Out}

The table below lists the 133 MIPs on which at least one of VDS-LCP or METHA found a feasible solution within the two hour time limit. It also lists the number of dives required to reach the feasible solution. If the number is in bold, that means that the method did not complete within the two hour time limit. If the number of dives is followed by a " ++ " the exact number of dives was not counted past that point due to either the method timing out, or the other branching variable selection method being deemed superior in terms of number of dives on that MIP.

\begin{tabular}{|l|r|r|}
\hline MIP & VDS-LCP Dives & METHA Dives \\
\hline 10teams.mps & 5 & 1 \\
\hline a1c1s1.mps & 1 & 1 \\
\hline aflow30a.mps & 1 & 4 \\
\hline aflow40b.mps & 1 & 1 \\
\hline air04.mps & 1 & 1 \\
\hline air05.mps & 1 & 1 \\
\hline atlanta-ip.mps & 9 & 754 \\
\hline bell3a.mps & 1 & 1 \\
\hline bell3b.mps & 1 & 1 \\
\hline bell4.mps & 1 & 1 \\
\hline bell5.mps & 1 & 1 \\
\hline bm23.mps & 16 & 1 \\
\hline cap6000.mps & 1 & 2 \\
\hline dano3mip.mps & 1 & 1 \\
\hline danoint.mps & 1 & 307 \\
\hline disktom.mps & 1 & 1 \\
\hline ds.mps & 1 & 1 \\
\hline fast0507.mps & 1 & 1 \\
\hline fiber.mps & 1 & 1 \\
\hline fixnet6.mps & 1 & 1 \\
\hline gesa2.mps & 1 & 1 \\
\hline gesa2-o.mps & 1 & 1 \\
\hline glass4.mps & 2 & 1 \\
\hline gt2.mps & 1 & 1 \\
\hline harp2.mps & 1 & 1 \\
\hline liu.mps & 1 & $20733++$ \\
\hline Iseu.mps & 1 & 1 \\
\hline manna81.mps & 1 & 1 \\
\hline markshare1.mps & & 1 \\
\hline & 1 & 1 \\
\hline
\end{tabular}




\begin{tabular}{|l|r|r|}
\hline markshare2.mps & 1 & 1 \\
\hline mas74.mps & 1 & 1 \\
\hline mas76.mps & 1 & 1 \\
\hline misc07.mps & 1 & 1 \\
\hline mkc.mps & 1 & 1 \\
\hline mod008.mps & 1 & 1 \\
\hline mod011.mps & 1 & 1 \\
\hline modglob.mps & 1 & 1 \\
\hline momentum1.mps & 15 & 9 \\
\hline mzzv11.mps & 11849 & 1 \\
\hline mzzv42z.mps & 1 & 1 \\
\hline neos-1056905.mps & 1 & 1 \\
\hline neos-1122047.mps & 1 & 1 \\
\hline neos-1171448.mps & 1 & 1 \\
\hline neos-1171692.mps & 1 & 1 \\
\hline neos-1171737.mps & 1 & 1 \\
\hline neos-1200887.mps & 1 & 1 \\
\hline neos-1211578.mps & 1 & 1 \\
\hline neos-1228986.mps & 1 & 1 \\
\hline neos13.mps & 1 & 1 \\
\hline neos-1311124.mps & 1 & 1 \\
\hline neos-1337489.mps & 1 & 1 \\
\hline neos-1346382.mps & 1 & 1 \\
\hline neos-1420205.mps & 1 & 1 \\
\hline neos-1426635.mps & 1 & 1 \\
\hline neos-1426662.mps & 1 & 1 \\
\hline neos-1427181.mps & 1 & 1 \\
\hline neos-1427261.mps & 1 & 1 \\
\hline neos-1429185.mps & 1 & 1 \\
\hline neos-1429461.mps & 1 & 1 \\
\hline neos-1430701.mps & 1 & 1 \\
\hline neos-1436709.mps & 1 & 1 \\
\hline neos-1436713.mps & 1 & 1 \\
\hline neos-1439395.mps & 1 & 1 \\
\hline neos-1440447.mps & 1 & 1 \\
\hline neos-1440457.mps & 1 & 1 \\
\hline neos-1440460.mps & 1 & 1 \\
\hline neos-1442119.mps & 1 & 1 \\
\hline neos-1442657.mps & 1 & 1 \\
\hline neos-1467067.mps & 1 & 1 \\
\hline neos-1480121.mps & 1 & 1 \\
\hline neos-1516309.mps & 1 & 1 \\
\hline neos-1599274.mps & 1 & 1 \\
\hline neos-1616732.mps & 1 & 1 \\
\hline neos-495307.mps & 1 & 1 \\
\hline neos5.mps & 1 & 1 \\
\hline neos-544324.mps & 1 & 1 \\
\hline neos-547911.mps & 1 & 1 \\
\hline
\end{tabular}




\begin{tabular}{|l|r|r|}
\hline neos-565672.mps & 1 & 1 \\
\hline neos-702280.mps & 1 & 1 \\
\hline neos-785914.mps & 1 & 155 \\
\hline neos-787933.mps & 1 & 1 \\
\hline neos-841664.mps & 1 & 1 \\
\hline neos-856059.mps & 1 & 1 \\
\hline neos-872648.mps & 1 & 1 \\
\hline neos-873061.mps & 1 & 1 \\
\hline neos-885086.mps & 1 & 1 \\
\hline neos-886822.mps & 1 & 1 \\
\hline neos-932721.mps & 1 & 1 \\
\hline neos-932816.mps & 1 & 1 \\
\hline neos-933638.mps & 1 & 1 \\
\hline neos-933966.mps & 1 & 1 \\
\hline neos-934278.mps & 1 & 1 \\
\hline neos-934441.mps & 1 & 1 \\
\hline neos-953928.mps & 1 & 1 \\
\hline neos-956971.mps & 1 & 1 \\
\hline neos-957143.mps & 1 & 1 \\
\hline neos-957323.mps & 1 & 1 \\
\hline noswot.mps & 1 & 1 \\
\hline nsrand-ipx.mps & 1 & 1 \\
\hline nw04.mps & 1 & 1 \\
\hline opt1217.mps & 1 & 1 \\
\hline p0033.mps & 1 & 1 \\
\hline p0040.mps & 1 & 1 \\
\hline p0201.mps & 1 & 1 \\
\hline p0282.mps & 1 & 1 \\
\hline p0291.mps & 1 & 1 \\
\hline p0548.mps & 1 & 1 \\
\hline p2756.mps & 1 & 1 \\
\hline pk1.mps & 1 & 1 \\
\hline pp08a.mps & 1 & 1 \\
\hline pp08aCUTS.mps & 1 & 1 \\
\hline protfold.mps & 1 & 1 \\
\hline qiu.mps & 1 & 1 \\
\hline roll3000.mps & 1 & 1 \\
\hline rout.mps & 1 & 1 \\
\hline sentoy.mps & 1 & 1 \\
\hline set1ch.mps & 1 & 1 \\
\hline seymour.disj- & 1 & 1 \\
\hline 0.mps & 1 & 1 \\
\hline seymour.mps & 1 & 1 \\
\hline sp97ic.mps & 1 & 1 \\
\hline sp98ar.mps & 1 & 1 \\
\hline sp98ic.mps & 1 & 1 \\
\hline sp98ir.mps & 1 & 1 \\
\hline & 1 & 1 \\
\hline
\end{tabular}




\begin{tabular}{|l|r|r|}
\hline stein27.mps & 1 & 1 \\
\hline stein45.mps & 1 & 1 \\
\hline swath.mps & 1 & 1 \\
\hline t1717.mps & 1 & 1 \\
\hline timtab1.mps & $5590++$ & $\mathbf{8 2 4 0 + +}$ \\
\hline timtab2.mps & 3 & $24046++$ \\
\hline tr12-30.mps & 1 & 1 \\
\hline vpm2.mps & 1 & 1 \\
\hline
\end{tabular}




\section{A.6 Constraint and Variable Analysis on the MIP Set}

The below table includes the number of times an integer or binary variable has a positive or negative coefficient in a less-than or greater-than inequality constraint for each of the 146 MIPs in the MIP set. The column \#VarsPL means the number of times an integer or binary variable has a positive $(\mathrm{P})$ coefficient in a less-than $(\mathrm{L})$ inequality. The \#VarsPL and the \#VarsNG columns represent the violated counts; whereas the \#VarsNL and \#VarsPG represent the satisfied counts. The pre-solver in GLPK actually converts the greater-than inequality constraints to less-than inequalities with opposite signs on the variable coefficients. This means there were no instances of integer or binary variables present in a greater-than inequality in the MIP set. For this reason the columns \#VarsPG and \#VarsNG were omitted from the table.

The columns PL Win and NL Win are binary. PL Win is set to 1 when there are more variables in the PL column than in the NL column for a particular MIP. If there is a tie (same number of variable instances in PL and NL), the Tie column is assigned a 1. The sums of these last three columns are where the percentages discussed in Section 9.2 are derived.

\begin{tabular}{|l|r|r|r|r|r|}
\hline MIP & \#VarsPL & \multicolumn{1}{l|}{ \#VarsNL } & \multicolumn{1}{l|}{ PL Win } & \multicolumn{1}{l|}{ NL Win } & \multicolumn{1}{l|}{ Tie } \\
\hline 10teams.mps & 4800 & 0 & 1 & 0 & 0 \\
\hline a1c1s1.mps & 176 & 960 & 0 & 1 & 0 \\
\hline aflow30a.mps & 0 & 421 & 0 & 1 & 0 \\
\hline aflow40b.mps & 0 & 1364 & 0 & 1 & 0 \\
\hline air04.mps & 0 & 0 & 0 & 0 & 1 \\
\hline air05.mps & 0 & 0 & 0 & 0 & 1 \\
\hline arki001.mps & 5667 & 1824 & 1 & 0 & 0 \\
\hline atlanta-ip.mps & 188818 & 8392 & 1 & 0 & 0 \\
\hline bell3a.mps & 57 & 72 & 0 & 1 & 0 \\
\hline bell3b.mps & 56 & 71 & 0 & 1 & 0 \\
\hline bell4.mps & 58 & 72 & 0 & 1 & 0 \\
\hline bell5.mps & 56 & 71 & 0 & 1 & 0 \\
\hline bm23.mps & 253 & 225 & 1 & 0 & 0 \\
\hline
\end{tabular}




\begin{tabular}{|c|c|c|c|c|c|}
\hline cap6000.mps & 17806 & 141 & 1 & 0 & 0 \\
\hline dano3mip.mps & 63 & 5517 & 0 & 1 & 0 \\
\hline danoint.mps & 0 & 560 & 0 & 1 & 0 \\
\hline disktom.mps & 0 & 0 & 0 & 0 & 1 \\
\hline ds.mps & 0 & 0 & 0 & 0 & 1 \\
\hline fast0507.mps & 0 & 407720 & 0 & 1 & 0 \\
\hline fiber.mps & 0 & 0 & 0 & 0 & 1 \\
\hline fixnet6.mps & 0 & 378 & 0 & 1 & 0 \\
\hline gesa2.mps & 1320 & 1968 & 0 & 1 & 0 \\
\hline gesa2-o.mps & 720 & 1080 & 0 & 1 & 0 \\
\hline glass4.mps & 347 & 0 & 1 & 0 & 0 \\
\hline gt2.mps & 181 & 173 & 1 & 0 & 0 \\
\hline harp2.mps & 860 & 367 & 1 & 0 & 0 \\
\hline liu.mps & 3135 & 3135 & 0 & 0 & 1 \\
\hline Iseu.mps & 113 & 195 & 0 & 1 & $\underline{0}$ \\
\hline manna81.mps & 12960 & 0 & 1 & 0 & 0 \\
\hline markshare1.mps & 0 & 0 & 0 & 0 & 1 \\
\hline markshare2.mps & 0 & 0 & 0 & 0 & 1 \\
\hline mas74.mps & 150 & 1544 & 0 & 1 & 0 \\
\hline mas76.mps & 150 & 1479 & 0 & 1 & 0 \\
\hline misc07.mps & 1001 & 6764 & 0 & 1 & 0 \\
\hline mkc.mps & 10814 & 2879 & 1 & 0 & 0 \\
\hline mod008.mps & 0 & 1243 & 0 & 1 & 0 \\
\hline mod011.mps & 96 & 96 & 0 & 0 & 1 \\
\hline modglob.mps & 0 & 196 & 0 & 1 & 0 \\
\hline momentum1.mps & 36104 & 3310 & 1 & 0 & 0 \\
\hline momentum2.mps & 19615 & 74950 & 0 & 1 & 0 \\
\hline momentum3.mps & 55233 & 12953 & 1 & 0 & 0 \\
\hline msc98-ip.mps & 51615 & 9126 & 1 & 0 & 0 \\
\hline mzzv11.mps & 113417 & 701 & 1 & 0 & 0 \\
\hline mzzv42z.mps & 127300 & 439 & 1 & 0 & 0 \\
\hline neos-1056905.mps & 840 & 840 & 0 & 0 & 1 \\
\hline neos-1122047.mps & 47960 & 100 & 1 & 0 & 0 \\
\hline neos-1171448.mps & 16002 & 2457 & 1 & 0 & 0 \\
\hline neos-1171692.mps & 4326 & 819 & 1 & 0 & 0 \\
\hline neos-1171737.mps & 3450 & 1170 & 1 & 0 & 0 \\
\hline neos-1200887.mps & 588 & 117 & 1 & 0 & 0 \\
\hline neos-1211578.mps & 295 & 130 & 1 & 0 & 0 \\
\hline neos-1228986.mps & 295 & 130 & 1 & 0 & 0 \\
\hline neos13.mps & 1815 & 1815 & 0 & 0 & 1 \\
\hline neos-1311124.mps & 1449 & 546 & 1 & 0 & 0 \\
\hline neos-1337489.mps & 295 & 130 & 1 & 0 & 0 \\
\hline neos-1346382.mps & 690 & 260 & 1 & 0 & 0 \\
\hline neos-1420205.mps & 630 & 420 & 1 & 0 & 0 \\
\hline neos-1426635.mps & 690 & 260 & 1 & 0 & 0 \\
\hline neos-1426662.mps & 1664 & 416 & 1 & 0 & 0 \\
\hline neos $-1427181 . \mathrm{mps}$ & 1408 & 416 & 1 & 0 & 0 \\
\hline neos-1427261.mps & 1760 & 520 & 1 & 0 & 0 \\
\hline
\end{tabular}




\begin{tabular}{|c|c|c|c|c|c|}
\hline neos-1429185.mps & 1056 & 312 & 1 & 0 & 0 \\
\hline neos-1429461.mps & 790 & 260 & 1 & 0 & 0 \\
\hline neos-1430701.mps & 474 & 156 & 1 & 0 & 0 \\
\hline neos-1436709.mps & 1027 & 338 & 1 & 0 & 0 \\
\hline neos-1436713.mps & 2112 & 624 & 1 & 0 & 0 \\
\hline neos-1439395.mps & 553 & 182 & 1 & 0 & 0 \\
\hline neos-1440447.mps & 395 & 130 & 1 & 0 & 0 \\
\hline neos-1440457.mps & 1422 & 468 & 1 & 0 & 0 \\
\hline neos-1440460.mps & 711 & 234 & 1 & 0 & 0 \\
\hline neos-1442119.mps & 1106 & 364 & 1 & 0 & 0 \\
\hline neos-1442657.mps & 948 & 312 & 1 & 0 & 0 \\
\hline neos-1467067.mps & 1449 & 598 & 1 & 0 & 0 \\
\hline neos-1480121.mps & 140 & 70 & 1 & 0 & 0 \\
\hline neos-1516309.mps & 12600 & 16900 & 0 & 1 & 0 \\
\hline neos-1599274.mps & 28350 & 16500 & 1 & 0 & 0 \\
\hline neos-1616732.mps & 0 & 3998 & 0 & 1 & 0 \\
\hline neos-495307.mps & 0 & 27831 & 0 & 1 & 0 \\
\hline neos $5 . \mathrm{mps}$ & 0 & 1696 & 0 & 1 & 0 \\
\hline neos-544324.mps & 883680 & 873600 & 1 & 0 & 0 \\
\hline neos-547911.mps & 309288 & 305760 & 1 & 0 & 0 \\
\hline neos-565672.mps & 318333 & 175079 & 1 & 0 & 0 \\
\hline neos-702280.mps & 0 & 2421882 & 0 & 1 & 0 \\
\hline neos-785899.mps & 12380 & 4800 & 1 & 0 & 0 \\
\hline neos-785912.mps & 12830 & 3780 & 1 & 0 & 0 \\
\hline neos-785914.mps & 11870 & 3420 & 1 & 0 & 0 \\
\hline neos-787933.mps & 234612 & 63708 & 1 & 0 & 0 \\
\hline neos-841664.mps & 0 & 2730 & 0 & 1 & 0 \\
\hline neos-856059.mps & 0 & 35654 & 0 & 1 & 0 \\
\hline neos-872648.mps & 0 & 87575 & 0 & 1 & 0 \\
\hline neos-873061.mps & 0 & 87644 & 0 & 1 & 0 \\
\hline neos-885086.mps & 7290 & 2430 & 1 & 0 & 0 \\
\hline neos-885524.mps & 87503 & 35290 & 1 & 0 & 0 \\
\hline neos-886822.mps & 2048 & 2048 & 0 & 0 & 1 \\
\hline neos-932721.mps & 37155 & 12870 & 1 & 0 & 0 \\
\hline neos-932816.mps & 263928 & 10304 & 1 & 0 & 0 \\
\hline neos-933638.mps & 48014 & 10579 & 1 & 0 & 0 \\
\hline neos-933966.mps & 42769 & 9269 & 1 & 0 & 0 \\
\hline neos-934278.mps & 43400 & 9599 & 1 & 0 & 0 \\
\hline neos-934441.mps & 44528 & 9862 & 1 & 0 & 0 \\
\hline neos-948346.mps & 459839 & 80604 & 1 & 0 & 0 \\
\hline neos-953928.mps & 129806 & 40055 & 1 & 0 & 0 \\
\hline neos-954925.mps & 696795 & 148188 & 1 & 0 & 0 \\
\hline neos-956971.mps & 381507 & 102053 & 1 & 0 & 0 \\
\hline neos-957143.mps & 415145 & 82531 & 1 & 0 & 0 \\
\hline neos-957323.mps & 416135 & 83521 & 1 & 0 & 0 \\
\hline net12.mps & 51656 & 576 & 1 & 0 & 0 \\
\hline noswot.mps & 373 & 128 & 1 & 0 & 0 \\
\hline nsrand-ipx.mps & 13240 & 205500 & 0 & 1 & 0 \\
\hline
\end{tabular}




\begin{tabular}{|c|c|c|c|c|c|}
\hline nw04.mps & 0 & 0 & 0 & 0 & 1 \\
\hline opt1217.mps & 0 & 758 & 0 & 1 & 0 \\
\hline p0033.mps & 32 & 65 & 0 & 1 & 0 \\
\hline p0040.mps & 40 & 70 & 0 & 1 & 0 \\
\hline p0201.mps & 375 & 1482 & 0 & 1 & 0 \\
\hline p0282.mps & 420 & 864 & 0 & 1 & 0 \\
\hline p0291.mps & 114 & 235 & 0 & 1 & 0 \\
\hline p0548.mps & 758 & 691 & 1 & 0 & 0 \\
\hline p2756.mps & 5033 & 3606 & 1 & 0 & 0 \\
\hline pk1.mps & 0 & 0 & 0 & 0 & 1 \\
\hline pp08a.mps & 0 & 64 & 0 & 1 & 0 \\
\hline pp08aCUTS.mps & 0 & 174 & 0 & 1 & 0 \\
\hline protfold.mps & 7701 & 12360 & 0 & 1 & 0 \\
\hline qiu.mps & 0 & 528 & 0 & 1 & 0 \\
\hline ramos3.mps & 0 & 32805 & 0 & 1 & 0 \\
\hline rd-rplusc-21.mps & 363099 & 120 & 1 & 0 & 0 \\
\hline roll3000.mps & 4540 & 12684 & 0 & 1 & 0 \\
\hline rout.mps & 615 & 240 & 1 & 0 & 0 \\
\hline sentoy.mps & 1800 & 0 & 1 & 0 & 0 \\
\hline set1ch.mps & 0 & 235 & 0 & 1 & 0 \\
\hline $\begin{array}{l}\text { seymour.disj- } \\
\text { 10.mps }\end{array}$ & 0 & 64704 & 0 & 1 & 0 \\
\hline seymour.mps & 0 & 33432 & 0 & 1 & 0 \\
\hline sp97ar.mps & 14079 & 276867 & 0 & 1 & 0 \\
\hline sp97ic.mps & 4278 & 48967 & 0 & 1 & 0 \\
\hline sp98ar.mps & 19401 & 191806 & 0 & 1 & 0 \\
\hline sp98ic.mps & 9072 & 113446 & 0 & 1 & 0 \\
\hline sp98ir.mps & 6430 & 61357 & 0 & 1 & 0 \\
\hline stein 15.mps & 0 & 120 & 0 & 1 & 0 \\
\hline stein27.mps & 0 & 378 & 0 & 1 & 0 \\
\hline stein45.mps & 0 & 1034 & 0 & 1 & 0 \\
\hline stp3d.mps & 244985 & 112973 & 1 & 0 & 0 \\
\hline swath.mps & 6080 & 0 & 1 & 0 & 0 \\
\hline t1717.mps & 0 & 0 & 0 & 0 & 1 \\
\hline timtab1.mps & 0 & 0 & 0 & 0 & 1 \\
\hline timtab2.mps & 0 & 0 & 0 & 0 & 1 \\
\hline tr12-30.mps & 352 & 352 & 0 & 0 & 1 \\
\hline vpm2.mps & 120 & 120 & 0 & 0 & 1 \\
\hline Sums & & & 74 & 53 & 19 \\
\hline Percentages & & & $51 \%$ & $36 \%$ & $13 \%$ \\
\hline
\end{tabular}

